\title{
Atlas of marine bony fish otoliths (sagittae) of Southeastern-Southern Brazil Part V: Perciformes (Sparidae, Sciaenidae, Polynemidae, Mullidae, Kyphosidae, Chaetodontidae, Mugilidae, Scaridae, Percophidae, Pinguipedidae, Blenniidae, Gobiidae, Ephippidae, Sphyraenidae, Gempylidae, Trichiuridae, Scombridae, Ariommatidae, Stromateidae and Caproidae)
}

Cesar Santificetur ${ }^{1}$, Valéria Regina Martins Conversani ${ }^{1}$, Marina Rito Brenha-Nunes ${ }^{1}$, Marcella Bockis Giarettal, Carolina Correia Siliprandi , Carmen Lucia Del Bianco Rossi-Wongtschowski ${ }^{l, *}$

${ }^{1}$ Instituto Oceanográfico da Universidade de São Paulo

(Praça do Oceanográfico, 191, CEP: 05508-120, São Paulo, SP, Brazil)

*Corresponding author: cwongski@usp.br

\section{Abstract}

This publication is part of a series prepared with the purpose to constitute an Atlas of Teleostei Otoliths for the Southeastern-Southern Brazilian area. Here we present the results of 15 morphological features and six shape indices for 33 Perciformes species of 20 families. Whenever available in out collection, three otoliths of each species were illustrated and photographed. The frequency of occurrence of each feature was calculated inside and among total length classes being the differences analyzed through multiple $\chi 2$ tests (significance level 0.05). Based on otoliths measurements, six shape indices values were obtained being the minimum, maximum, mean and standard deviations values presented.

Descriptors: Otoliths, Morphology, Morphometry, Southwestern Atlantic, Brazil, Perciformes.

\section{Resumo}

Esta publicação é a continuação de uma série que deverá resultar em um Atlas de Otólitos de Teleósteos da Região Sudeste-Sul brasileira. Aqui apresentamos os resultados de análises morfológicas relativas a 15 características e seis índices usualmente utilizados para a caracterização dessas estruturas. Neste estudo são apresentados os resultados obtidos para 33 espécies de Percifomes de 20 famílias. Sempre que possível, foram desenhados e fotografados três otólitos de cada espécie. A frequência de ocorrência de cada característica morfológica foi calculada por classes de comprimento total (TL) e para toda a amostra, sendo as diferenças, dentro de cada classe e, ao longo do desenvolvimento do peixe, analisadas por meio de testes de $\chi^{2}$ múltiplo (nível de significância 0,05). A partir de medidas dos otólitos, foram calculados valores de seis índices de forma, sendo aqui apresentados seus valores mínimo, máximo, média e desvio padrão.

Descritores: Otólitos, Morfologia, Morfometria, Atlântico Sudoeste, Brasil, Perciformes.

\section{INTRODUCTION}

Many papers have been published showing the importance of the otoliths as indicators for community, population, individual analysis and environmental events.

As a contribution for these studies we have been producing several papers about the shape of these structures since their specific variation is useful for studies on taxonomy, phylogeny, archeology, paleontology, species geographic variation, stock identification, food webs and others.

The basic material for our studies comes from the Collection of Otoliths of Teleostei Fish of Southeastern-Southern Brazil (COSS-Brasil), held at the Laboratory of Ichthyofauna and Growth (Laboratório de Ictiofauna e Crescimento LABIC) of Instituto Oceanográfico, (Universidade de São Paulo - Brasil), presently containing 51886 otoliths corresponding to 201 different species (ROSSI-WONGTSCHOWSKI et al., 2016).

This publication contains the results of fifteen morphological data and six shape indices of the mostly common features for 33 Perciformes species.

Submitted on: 18/07/2016

Approved on: 22/11/2016

http://dx.doi.org/10.1590/S1679-87592017131006502 
At the end of these publications we intend to constitute an Atlas of Teleostei Otoliths for the Southeastern-Southern Brazilian region.

\section{MATERIAL AND METHODS}

The sampled area and the methodology of this study followed that presented in ROSSI-WONGTSCHOWSKI et al. (2014), SILIPRANDI et al. (2016) and BRENHA-NUNES et al. (2016).

The acronyms presented in the shape indices tables are: $\mathrm{TL}=$ total fish length, $\mathrm{OL}=$ otolith length, $\mathrm{OH}=\mathrm{otolith}$ height and $\mathrm{OT}=$ otolith thickness.

\section{RESULTS}

\section{PART V: PERCIFORMES}

\section{FAMILY SPARIDAE}

The sulcus acusticus opening is ostial, the ostium is funnel-like and the cauda is frequently tubular strongly curved; the profile is concave-convex; the pseudorostrum and pseudoantirostrum are always absent and the rostrum is developed in mostly cases.

Archosargus rhomboidalis (Linnaeus 1758) Plate 1

\begin{tabular}{ll}
\hline Maximum Size: & $355 \mathrm{~mm}$ (TL) (MENEZES; FIGUEIREDO, 1980) \\
Distribution: & Western Atlantic, from New Jersey and Northeastern Gulf of Mexico to Southeastern Brazil (MENEZES et al., 2003) \\
Habitat: & Coastal waters over mud or sand bottoms, occasionally found in coral reef and rocky areas (MENEZES; FIGUEIREDO, 1980) \\
Diet: & Mainly benthic invertebrates (mollusks and crustaceans); adults feed mostly on algae (VAUGHAN, 1978) \\
Collection: & 77 otoliths from 39 fish (TL ranging from 115 to $160 \mathrm{~mm})$ \\
Sample: & 22 left otoliths categorized into 4, $20 \mathrm{~mm}$ classes (100 to $160 \mathrm{~mm}$ ) \\
\hline
\end{tabular}

Shape: elliptic (77\%), elliptic to triangular. Anterior region: peaked-round (41\%), oblique (36\%), angled-round. Posterior region: oblique-round (45\%), round (36\%), oblique. Dorsal edge: crenate to sinuate (50\%), sinuate to entire, lobed to sinuate, crenate to entire. Ventral edge: sinuate to entire. Profile: concave-convex. Rostrum and antirostrum orientation: does not apply (50\%), in agreement (50\%). Rostrum: developed (82\%), underdeveloped. Antirostrum: absent (50\%), underdeveloped, developed. Sulcus acusticus: position: median; orientation: horizontal; opening: ostial; morphology: heterosulcoid; colliculum: heteromorphic; ostium: funnel-like; cauda: tubular strongly curved (59\%), tubular markedly curved, tubular slightly curved.

Statistical differences $(p<0.05)$ within some length classes were obtained for shape, dorsal edge, rostrum and antirostrum orientation and development. Along the growth development, statistical differences were found for rostrum and antirostrum orientation and antirostrum development.

\begin{tabular}{lccc}
\hline Shape indices & Mean \pm Sd & Minimum & Maximum \\
\hline OL/TL (\%) & $2.94 \pm 0.22$ & 2.53 & 3.54 \\
OH/OL (\%) & $67.10 \pm 2.68$ & 62.44 & 71.85 \\
OT/OL (\%) & $16.58 \pm 1.27$ & 15.01 & 20.11 \\
OT/OH (\%) & $24.72 \pm 1.67$ & 21.77 & 27.99 \\
Circularity & $18.73 \pm 1.19$ & 16.80 & 20.67 \\
Rectangularity & $0.69 \pm 0.01$ & 0.67 & 0.71 \\
\hline
\end{tabular}


Calamus penna (Valenciennes 1830) Plate 2

\begin{tabular}{|c|c|}
\hline Maximum Size: & 500 mm (TL) (MENEZES; FIGUEIREDO, 1980) \\
\hline Distribution: & Western Atlantic from Florida and Bahamas to Southeastern Brazil (CARVALHO-FILHO, 1992; MENEZES et al., 2003) \\
\hline Habitat: & Shallow waters over rocky areas or reefs. Adults are often founds on flat bottoms (CARPENTER, 2002) \\
\hline Diet: & Mainly crustaceans, mollusks and urchins (CARVALHO-FILHO, 1992; CARPENTER, 2002) \\
\hline Collection: & 1 otolith from 1 fish (TL: $167 \mathrm{~mm}$ ) \\
\hline Sample: & 1 left otolith (167 mm) \\
\hline
\end{tabular}

Shape: triangular. Anterior region: peaked. Posterior region: peaked-round. Anterior dorsal edge: sinuate. Posterior dorsal edge: sinuate to entire. Ventral edge: sinuate. Profile: concave-convex. Rostrum and antirostrum orientation: in disagreement. Rostrum: developed. Antirostrum: developed. Sulcus acusticus: position: supramedian; orientation: ascending; opening: ostial; morphology: heterosulcoid; colliculum: heteromorphic; ostium: funnel-like; cauda: tubular strongly curved.

Only one otolith was examined not allowing statistical analysis of the data but its morphometric characteristics are shown below:

\begin{tabular}{lccc}
\hline Shape indices & Mean \pm Sd & Minimum & Maximum \\
\hline OL/TL (\%) & $3.43 \pm 0$ & 3.43 & 3.43 \\
OH/OL (\%) & $45.28 \pm 0$ & 45.28 & 45.28 \\
OT/OL (\%) & $15.91 \pm 0$ & 15.91 & 15.91 \\
OT/OH (\%) & $35.14 \pm 0$ & 35.14 & 35.14 \\
Circularity & $20.63 \pm 0$ & 20.63 & 20.63 \\
Rectangularity & $0.69 \pm 0$ & 0.69 & 0.69 \\
\hline
\end{tabular}

Pagrus pagrus (Linnaeus 1758) Plate 3

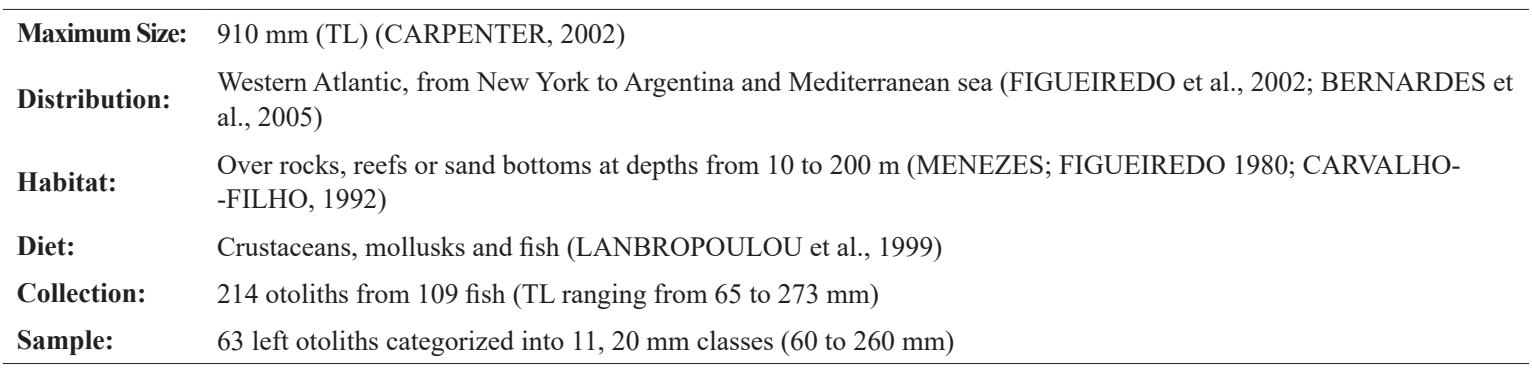

Shape: elliptic (51\%), elliptic to pentagonal (49\%). Anterior region: angled (68\%), peaked, angled-round, peakedround. Posterior region: angled (60\%), angled-round, round, oblique to angled. Anterior dorsal edge: does not apply (51\%), sinuate (43\%), dentate, lobed to sinuate, dentate to lobed. Posterior dorsal edge: does not apply (51\%), dentate, lobed, lobed to sinuate. Anterior ventral edge: lobed to sinuate (46\%), dentate to lobed, sinuate, lobed. Posterior ventral edge: lobed to sinuate (46\%), dentate to lobed, sinuate, lobed. Profile: concave-convex (83\%), plane-convex. Rostrum and antirostrum orientation: in agreement (56\%), does not apply, in disagreement. Rostrum: developed. Antirostrum: developed (41\%), absent (33\%), underdeveloped. Sulcus acusticus: position: supramedian (87\%), median; orientation: horizontal; opening: ostial; morphology: heterosulcoid; colliculum: heteromorphic; ostium: funnel-like; cauda: tubular strongly curved (52\%), tubular slightly curved (48\%).

Statistical differences $(p<0.05)$ within some length classes were obtained for shape, anterior and posterior regions, dorsal, posterior dorsal, ventral and posterior ventral edges, rostrum and antirostrum orientation, profile and sulcus acusticus position. Along the development statistical differences were found for shape, dorsal, posterior dorsal, ventral and posterior ventral edges, rostrum and antirostrum orientation, profile and sulcus acusticus position. 


\begin{tabular}{lccc}
\hline Shape indices & Mean \pm Sd & Minimum & Maximum \\
\hline OL/TL (\%) & $4.37 \pm 0.39$ & 3.64 & 5.33 \\
OH/OL (\%) & $73.30 \pm 3.85$ & 62.77 & 81.90 \\
OT/OL (\%) & $18.17 \pm 1.48$ & 14.78 & 22.29 \\
OT/OH (\%) & $24.84 \pm 2.23$ & 21.23 & 30.23 \\
Circularity & $19.17 \pm 1.78$ & 15.53 & 22.53 \\
Rectangularity & $0.66 \pm 0.02$ & 0.63 & 0.70 \\
\hline
\end{tabular}

\section{FAMIILY SCIAENIDAE}

Cynoscion leiarchus (Cuvier 1830) Plate 4

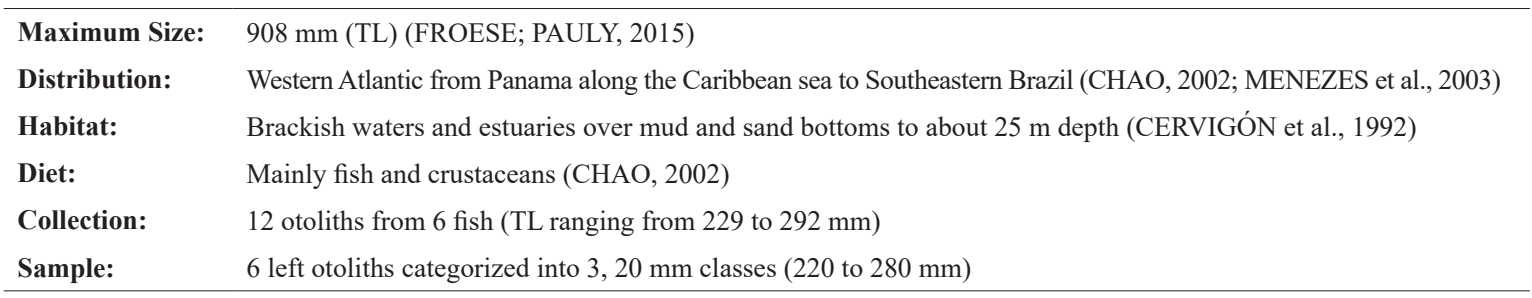

Shape: elliptic. Anterior region: round. Posterior region: angled-round. Anterior dorsal edge: entire (67\%), sinuate to entire. Ventral edge: sinuate to entire. Profile: biconvex. Rostrum and antirostrum orientation: does not apply. Rostrum: absent. Antirostrum: absent. Pseudorostrum and Pseudoantirostrum: absent. Sulcus acusticus: position: median; orientation: horizontal; opening: pseudo-ostial; morphology: heterosulcoid; colliculum: heteromorphic; ostium: discoidal; cauda: tubular markedly curved, tubular curled.

The small number of otoliths examined did not permit the statistical analysis of the data but their morphometric characteristics are shown below:

\begin{tabular}{lccc}
\hline Shape indices & Mean \pm Sd & Minimum & Maximum \\
\hline OL/TL (\%) & $4.79 \pm 0.19$ & 4.58 & 5.10 \\
OH/OL (\%) & $9.84 \pm 1.32$ & 48.70 & 52.23 \\
OT/OL (\%) & $23.38 \pm 1.08$ & 21.93 & 24.45 \\
OT/OH (\%) & $46.93 \pm 2.37$ & 43.69 & 49.74 \\
Circularity & $16.24 \pm 0.34$ & 15.63 & 16.68 \\
Rectangularity & $0.78 \pm 0.01$ & 0.77 & 0.79 \\
\hline
\end{tabular}

\section{FAMILY POLYNEMIDAE}

\section{Polydactylus virginicus (Linnaeus 1758) Plate 5}

\begin{tabular}{ll} 
Maximum Size: & $330 \mathrm{~mm}$ (TL) (FELTES, 2002) but attaining $352 \mathrm{~mm}$ in our collection. \\
Distribution: & Western Atlantic from Florida to Argentina (MENEZES et al., 2003) \\
Habitat: & Coastal waters, estuaries and mangroves over sandy and muddy bottoms (CARVALHO-FILHO, 1992) \\
Diet: & Mainly crustaceans, followed by chaetognaths, polychaetes and plant material (LOPES; OLIVEIRA-SILVA, 1998) \\
Collection: & 46 otoliths from 24 fish (TL ranging from 215 to $352 \mathrm{~mm})$ \\
Sample: & 12 left otoliths categorized into $6,20 \mathrm{~mm}$ classes (200 to $340 \mathrm{~mm}$ ) \\
\hline
\end{tabular}


Shape: oblong (67\%), oblong to rectangular. Anterior region: flattened (42\%), round (33\%), oblique-round. Posterior region: oblique (50\%), round, peaked-round. Dorsal edge: lobed to sinuate (83\%), sinuate to entire. Ventral edge: sinuate to entire (75\%), lobed to sinuate, entire. Profile: concave-convex. Rostrum and antirostrum orientation: in agreement. Rostrum: underdeveloped. Antirostrum: developed (58\%), underdeveloped. Pseudorostrum: absent. Pseudoantirostrum: absent. Sulcus acusticus: position: median; orientation: horizontal (67\%), descending; opening: ostial; morphology: heterosulcoid; colliculum: heteromorphic; ostium: funnel-like; cauda: tubular strongly curved (75\%), tubular markedly curved.

Statistical differences $(p<0.05)$ within some length classes were obtained for dorsal and ventral edges and sulcus acusticus orientation. No differences appeared during the fish's development.

\begin{tabular}{lccc}
\hline Shape indices & Mean \pm Sd & Minimum & Maximum \\
\hline OL/TL (\%) & $2.40 \pm 0.13$ & 2.19 & 2.73 \\
OH/OL (\%) & $49.17 \pm 1.88$ & 45.85 & 51.57 \\
OT/OL (\%) & $15.02 \pm 1.06$ & 13.44 & 16.80 \\
OT/OH (\%) & $30.57 \pm 2.10$ & 26.27 & 33.59 \\
Circularity & $21.38 \pm 1.70$ & 19.31 & 24.61 \\
Rectangularity & $0.71 \pm 0.03$ & 0.67 & 0.76 \\
\hline
\end{tabular}

\section{FAMIILY MULLIDAE}

Otolith shape is frequently elliptic to trapezoidal; the profile is concave-convex; the rostrum is developed; the pseudorostrum and pseudoantirostrum are always absent; the cauda is tubular markedly curved with the final region round and deeper.

\section{Mullus argentinae Hubbs \& Marini 1933 Plate 6}

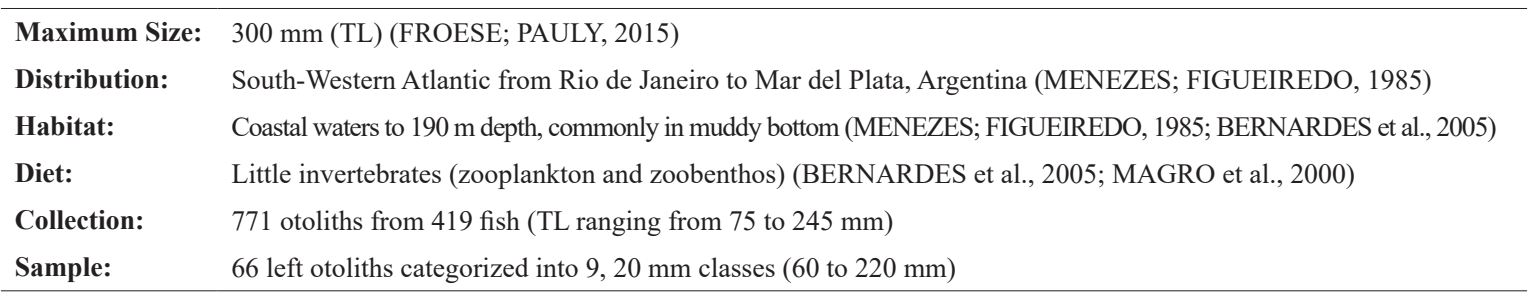

Shape: elliptic to trapezoidal (73\%), elliptic, fusiform. Anterior region: peaked (36\%), peaked-round (32\%), oblique, double-peaked. Posterior region: oblique (53\%), oblique to angled, angled, oblique-round. Dorsal edge: sinuate (55\%), lobed to sinuate, lobed, irregular. Ventral edge: lobed to sinuate (59\%), sinuate, lobed, irregular. Profile: concave-convex. Rostrum and antirostrum orientation: in agreement (98\%), does not apply. Rostrum: developed (98\%), underdeveloped. Antirostrum: developed (94\%), underdeveloped, absent. Sulcus acusticus: position: median; orientation: horizontal; opening: ostial; morphology: heterosulcoid; colliculum: heteromorphic; ostium: funnel-like; cauda: tubular markedly curved.

Statistical differences $(p<0.05)$ within some length classes were obtained for shape, anterior region, sulcus acusticus opening, rostrum and antirostrum orientation and development. Along the growth statistical differences were found only for the anterior region, rostrum and antirostrum orientation and development.

\begin{tabular}{lccc}
\hline Shape indices & Mean \pm Sd & Minimum & Maximum \\
\hline OL/TL (\%) & $2.31 \pm 0.43$ & 1.76 & 3.83 \\
OH/OL (\%) & $67.04 \pm 4.88$ & 55.12 & 81.38 \\
OT/OL (\%) & $18.90 \pm 1.74$ & 15.12 & 23.74 \\
OT/OH (\%) & $28.25 \pm 2.38$ & 24.35 & 33.67 \\
Circularity & $20.17 \pm 3.06$ & 16.25 & 39.26 \\
Rectangularity & $0.68 \pm 0.03$ & 0.56 & 0.72 \\
\hline
\end{tabular}


Upeneus parvus Poey 1852 Plate 7

\begin{tabular}{|c|c|}
\hline Maximum Size: & 300 mm (TL) (FROESE; PAULY, 2015) \\
\hline Distribution: & Western Atlantic from North Carolina to Santa Catarina state (MENEZES; FIGUEIREDO, 1985) \\
\hline Habitat: & Coastal waters from 25 to $112 \mathrm{~m}$ depth, live on mud-sandy bottom (MENEZES; FIGUEIREDO, 1985) \\
\hline Diet: & Mainly benthic invertebrates (FROESE; PAULY, 2015) \\
\hline Collection: & 148 otoliths from 79 fish (TL ranging from 81 to $175 \mathrm{~mm}$ ) \\
\hline Sample: & 39 left otoliths categorized into $5,20 \mathrm{~mm}$ classes ( 80 to $160 \mathrm{~mm}$ ) \\
\hline
\end{tabular}

Shape: elliptic (97\%), elliptic to trapezoidal. Anterior region: angled (49\%), peaked (44\%), oblique, double-peaked. Posterior region: angled-round (49\%), round, angled, oblique-round. Dorsal edge: lobed to sinuate (36\%), lobed, dentate to lobed, sinuate. Ventral edge: lobed to sinuate (59\%), lobed, dentate to lobed, sinuate. Profile: concave-convex. Rostrum and antirostrum orientation: does not apply (56\%), in agreement. Rostrum: developed (72\%), underdeveloped. Antirostrum: absent (49\%), underdeveloped (44\%), developed. Sulcus acusticus: position: median; orientation: horizontal; opening: ostial (95\%), ostio-caudal; morphology: heterosulcoid; colliculum: heteromorphic; ostium: funnel-like; cauda: tubular markedly curved.

Statistical differences $(p<0.05)$ within some length classes were obtained for shape, anterior region, sulcus acusticus opening, rostrum and antirostrum orientation and development. Along the growth, statistical differences were found for the anterior region, rostrum and antirostrum orientation and development.

\begin{tabular}{lccc}
\hline Shape indices & Mean \pm Sd & Minimum & Maximum \\
\hline OL/TL (\%) & $2.73 \pm 0.23$ & 2.31 & 3.54 \\
OH/OL (\%) & $65.80 \pm 2.58$ & 60.75 & 72.75 \\
OT/OL (\%) & $16.45 \pm 1.26$ & 14.23 & 20.00 \\
OT/OH (\%) & $25.03 \pm 1.94$ & 21.72 & 31.0 \\
Circularity & $17.91 \pm 1.67$ & 15.65 & 22.3 \\
Rectangularity & $0.70 \pm 0.02$ & 0.66 & 0.70 \\
\hline
\end{tabular}

\section{FAMILY KYPHOSIDAE}

Otolith shape is fusiform; the posterior region tends to be oblique-round; the profile is concave-convex; the rostrum and antirostrum are frequently developed and in agreement; the pseudorostrum and pseudoantirostrum are always absent; the ostium is funnel-like and the cauda is frequently tubular markedly curved.

\section{Kyphosus incisor (Cuvier 1831) Plate 8}

\begin{tabular}{|c|c|}
\hline Maximum Size: & 910 mm (TL) (FIGUEIREDO et al., 2002; BERNARDES et al., 2005) \\
\hline Distribution: & Western Atlantic from Virginia to Mar del Plata, Argentina (FIGUEIREDO et al., 2002; BERNARDES et al., 2005) \\
\hline Habitat: & Shallow waters on rocky bottom (BERNARDES et al., 2005) \\
\hline Diet: & Algae including Sargassum and some invertebrates (BERNARDES et al., 2005; SILVANO; GÜTH, 2006) \\
\hline Collection: & 2 otoliths from 1 fish (TL: $254 \mathrm{~mm}$ ) \\
\hline Sample: & 1 left otolith (254 mm) \\
\hline
\end{tabular}

Shape: fusiform. Anterior region: lanceolated-round. Posterior region: oblique-round. Dorsal edge: lobed to sinuate. Ventral edge: sinuate to entire. Profile: concave-convex. Rostrum and antirostrum orientation: in agreement. Rostrum: developed. Antirostrum: developed. Sulcus acusticus: position: median; orientation: horizontal; opening: ostial; morphology: heterosulcoid; colliculum: heteromorphic; ostium: funnel-like; cauda: tubular markedly curved. 
Since only one otolith was analyzed it was not possible statistical analysis of the data but its morphometric characteristics are shown below:

\begin{tabular}{lccc}
\hline Shape indices & Mean \pm Sd & Minimum & Maximum \\
\hline OL/TL (\%) & $2.72 \pm 0$ & 2.72 & 2.72 \\
OH/OL (\%) & $38.78 \pm 0$ & 38.78 & 38.78 \\
OT/OL (\%) & $11.00 \pm 0$ & 11.00 & 11.00 \\
OT/OH (\%) & $28.36 \pm 0$ & 28.36 & 28.36 \\
Circularity & $26.35 \pm 0$ & 26.35 & 26.35 \\
Rectangularity & $0.71 \pm 0$ & 0.71 & 0.71 \\
\hline
\end{tabular}

Kyphosus sectatrix (Linnaeus 1758) Plate 9

\begin{tabular}{ll}
\hline Maximum Size: & $760 \mathrm{~mm}$ (TL) (FROESE; PAULY, 2015). \\
Distribution: & Western Atlantic from Massachusetts and Bermuda to Santa Catarina state (MENEZES; FIGUEIREDO, 1985; \\
Habitat: & CARVALHO-FILHO, 1992) \\
Diet: & Benthic algae, zooplankton, crabs and mollusks (CARVALHO-FILHO, 1992; FROESE; PAULY, 2015) \\
Collection: & 28 otoliths from 15 fish (TL ranging from 25 to $454 \mathrm{~mm})$ \\
Sample: & 9 left otoliths categorized into $4,20 \mathrm{~mm}$ classes $(280$ to $340 \mathrm{~mm})$ \\
\hline
\end{tabular}

Shape: fusiform. Anterior region: round (89\%), peaked-round. Posterior region: oblique-round (78\%), peakedround. Dorsal edge: lobed to sinuate (56\%), crenate to sinuate, crenate to lobed. Ventral edge: crenate to sinuate (89\%), dentate to lobed. Profile: concave-convex. Rostrum and antirostrum orientation: in agreement. Rostrum: developed. Antirostrum: developed (78\%), underdeveloped. Sulcus acusticus: position: median; orientation: horizontal; opening: ostial; morphology: heterosulcoid; colliculum: heteromorphic; ostium: funnel-like; cauda: tubular markedly curved (78\%), tubular strongly curved.

The small number of otoliths examined did not permit the statistical analysis of the data but their morphometric characteristics are shown below:

\begin{tabular}{lccc}
\hline Shape indices & Mean \pm Sd & Minimum & Maximum \\
\hline OL/TL (\%) & $2.20 \pm 0.19$ & 1.76 & 2.40 \\
OH/OL (\%) & $44.61 \pm 1.71$ & 41.90 & 47.46 \\
OT/OL (\%) & $12.15 \pm 0.43$ & 11.60 & 12.91 \\
OT/OH (\%) & $27.28 \pm 1.59$ & 25.47 & 29.90 \\
Circularity & $25.72 \pm 2.38$ & 23.39 & 29.65 \\
Rectangularity & $0.71 \pm 0.02$ & 0.67 & 0.74 \\
\hline
\end{tabular}

\section{FAMILY CHAETODONTIDAE}

\section{Chaetodon striatus Linnaeus 1758 Plate 10}

\begin{tabular}{ll} 
Maximum Size: & $160 \mathrm{~mm}$ (TL) (FROESE; PAULY, 2015) \\
Distribution: & Eastern and Western Atlantic, from New Jersey to Santa Catarina state, Brazil (MENEZES; FIGUEIREDO, 1985) \\
Habitat: & Rocky bottoms and reef areas from coastal shelf to oceanic islands (BURGESS, 2002; CARVALHO-FILHO, 1992) \\
Diet: & Small benthic invertebrates, as coral polyps, crustaceans and mollusk eggs (BURGESS, 2002; FROESE; PAULY, 2015) \\
Collection: & 25 otoliths from 13 fish (TL ranging from 128 to $149 \mathrm{~mm})$ \\
Sample: & 8 left otoliths categorized into $2,20 \mathrm{~mm}$ classes (120 to $140 \mathrm{~mm})$ \\
\hline
\end{tabular}


Shape: elliptic. Anterior region: angled-round (38\%), peaked (38\%), peaked-round. Posterior region: round (63\%), oblique to angled, oblique, flattened. Dorsal edge: sinuate to entire (63\%), lobed to sinuate. Ventral edge: sinuate to entire. Profile: concave-convex. Rostrum and antirostrum orientation: in agreement. Rostrum: developed. Antirostrum: developed (50\%), underdeveloped (50\%). Pseudorostrum and Pseudoantirostrum: absent. Sulcus acusticus: position: median; orientation: descending (88\%), horizontal; opening: ostial; morphology: heterosulcoid; colliculum: heteromorphic; ostium: funnel-like; cauda: tubular sinuous (75\%), tubular strongly curved.

The small number of otoliths examined did not permit the statistical analysis of the data but their morphometric characteristics are shown below:

\begin{tabular}{lccc}
\hline Shape indices & Mean \pm Sd & Minimum & Maximum \\
\hline OL/TL (\%) & $3.43 \pm 0.21$ & 3.23 & 3.81 \\
OH/OL (\%) & $55.52 \pm 4.71$ & 49.57 & 63.05 \\
OT/OL (\%) & $18.93 \pm 1.81$ & 16.82 & 22.22 \\
OT/OH (\%) & $34.15 \pm 2.64$ & 30.53 & 38.19 \\
Circularity & $19.80 \pm 1.32$ & 17.59 & 21.92 \\
Rectangularity & $0.69 \pm 0.02$ & 0.66 & 0.74 \\
\hline
\end{tabular}

\section{FAMILY MUGILIDAE}

Otolith shape is oblong; the posterior region is round; the antirostrum is absent in mostly cases; the pseudorostrum and pseudoantirostrum are always absent; sulcus acusticus position supramedial and orientation ascending.

\section{Mugil curema Valenciennes 1836 Plate 11}

\begin{tabular}{ll}
\hline Maximum Size: & $910 \mathrm{~mm}$ (TL) (HARRISON, 2002) \\
Distribution: & Eastern Atlantic from Senegal River to the Congo River; Western Atlantic, from Nova Scotia to Argentina (HAR- \\
& RISON, 2002; FROESE; PAULY, 2015) \\
Habitat: & Inshore waters and estuaries with mud-sandy bottom (HARRISON, 2002; FROESE; PAULY, 2015) \\
Diet: & Organic detritus and small particulate materials (HARRISON, 2002) \\
Collection: & 1231 otoliths from 642 fish (TL ranging from 21 to $452 \mathrm{~mm})$ \\
Sample: & 117 left otoliths categorized into $20,20 \mathrm{~mm}$ classes $(20$ to $420 \mathrm{~mm})$ \\
\hline
\end{tabular}

Shape: oblong (88\%), elliptic. Anterior region: angled-round (42\%), peaked, angled, double-peaked. Posterior region: round (88\%), oblique-round, angled-round. Dorsal edge: entire (44\%), sinuate to entire, dentate to lobed, lobed to sinuate. Ventral edge: sinuate to entire (53\%), dentate to lobed, lobed to sinuate, lobed. Profile: concave-convex. Rostrum and antirostrum orientation: does not apply (64\%), in agreement. Rostrum: developed (53\%), underdeveloped, absent. Antirostrum: absent (64\%), underdeveloped, developed. Sulcus acusticus: position: supramedian (98\%), median; orientation: ascending (94\%), horizontal; opening: ostial; morphology: heterosulcoid; colliculum: heteromorphic; ostium: funnel-like (79\%), elliptic; cauda: tubular sinuous (85\%), tubular slightly curved, tubular strongly curved.

Statistical differences $(p<0.05)$ within some length classes were obtained for shape, anterior and posterior regions, dorsal and ventral edges, rostrum and antirostrum orientation and development, sulcus acusticus opening, position and orientation and ostium and cauda morphology. Along the development statistical differences were found for shape, anterior region, dorsal and ventral edges, antirostrum development, rostrum and antirostrum orientation and ostium and cauda morphology. 


\begin{tabular}{lccc}
\hline Shape indices & Mean \pm Sd & Minimum & Maximum \\
\hline OL/TL (\%) & $3.65 \pm 0.82$ & 2.14 & 5.61 \\
OH/OL (\%) & $52.29 \pm 6.29$ & 41.69 & 70.90 \\
OT/OL (\%) & $14.99 \pm 2.21$ & 11.35 & 23.28 \\
OT/OH (\%) & $28.68 \pm 2.42$ & 22.78 & 35.06 \\
Circularity & $21.38 \pm 4.15$ & 14.82 & 39.66 \\
Rectangularity & $0.71 \pm 0.04$ & 0.46 & 0.78 \\
\hline
\end{tabular}

\section{Mugil liza Valenciennes 1836 Plate 12}

\begin{tabular}{ll}
\hline Maximum Size: & $1000 \mathrm{~mm}$ (TL) (HARRISON, 2002; MENEZES; FIGUEIREDO, 1985) \\
Distribution: & Western Atlantic from Bermudas to Rio de Janeiro state (MENEZES; FIGUEIREDO, 1985) \\
Habitat: & Inshore marine waters and brackish estuaries (HARRISON, 2002; FROESE; PAULY, 2015) \\
Diet: & Organic detritus and filamentous algae (FROESE; PAULY, 2015) \\
Collection: & 4 otoliths from 2 fish (TL ranging from 373 to $485 \mathrm{~mm})$ \\
Sample: & 2 left otoliths categorized into 2, $20 \mathrm{~mm}$ classes (360 to $480 \mathrm{~mm}$ ) \\
\hline
\end{tabular}

Shape: oblong. Anterior region: peaked. Posterior region: round. Dorsal edge: sinuate (50\%), lobed to entire (50\%). Ventral edge: crenate to irregular (50\%), crenate to lobed (50\%). Profile: concave-convex. Rostrum and antirostrum orientation: does not apply. Rostrum: developed. Antirostrum: absent. Sulcus acusticus: position: supramedian; orientation: ascending; opening: ostial; morphology: heterosulcoid; colliculum: heteromorphic; ostium: funnel-like; cauda: tubular slightly curved.

The small number of otoliths examined did not allow statistical analysis of the data but their morphometric characteristics are shown below:

\begin{tabular}{lccc}
\hline Shape indices & Mean \pm Sd & Minimum & Maximum \\
\hline OL/TL (\%) & $2.26 \pm 0.25$ & 2.09 & 2.44 \\
OH/OL (\%) & $44.98 \pm 4.16$ & 42.04 & 47.92 \\
OT/OL (\%) & $13.32 \pm 0.11$ & 13.24 & 13.39 \\
OT/OH (\%) & $29.74 \pm 2.99$ & 27.63 & 31.85 \\
Circularity & $26.41 \pm 1.82$ & 25.13 & 27.70 \\
Rectangularity & $0.68 \pm 0$ & 0.68 & 0.68 \\
\hline
\end{tabular}

\section{FAMILY SCARIDAE}

Nicholsina usta (Valenciennes 1840) Plate 13

\begin{tabular}{ll} 
Maximum Size: & $300 \mathrm{~mm}$ (TL) (WESTNEAT, 2002; FROESE; PAULY, 2015) \\
Distribution: & Western Atlantic from New Jersey to São Paulo state, Brazil (MENEZES; FIGUEIREDO, 1985) \\
Habitat: & Shallow waters and seagrass beds (WESTNEAT, 2002; FROESE; PAULY, 2015) \\
Diet: & Herbivorous, feeding on seagrass (WESTNEAT, 2002) \\
Collection: & 5 otoliths from 3 fish (TL ranging from 148 to $175 \mathrm{~mm})$ \\
Sample: & 3 left otoliths categorized into 2, $20 \mathrm{~mm}$ classes (140 to $160 \mathrm{~mm}$ ) \\
\hline
\end{tabular}


Shape: elliptic (67\%), elliptic to rectangular. Anterior region: peaked (33\%), double-peaked-round (33\%), angledround (33\%). Posterior region: oblique-round (67\%), round. Dorsal edge: lobed to sinuate. Ventral edge: dentate to lobed (67\%), lobed. Profile: concave-convex. Rostrum and antirostrum orientation: in agreement (67\%), in disagreement. Rostrum: developed. Antirostrum: developed. Pseudorostrum and Pseudoantirostrum: absent. Sulcus acusticus: position: supramedian; orientation: horizontal; opening: ostial; morphology: heterosulcoid; colliculum: heteromorphic; ostium: funnel-like (67\%), round-oval; cauda: elliptic.

The small number of otoliths examined did not permit the statistical analysis of the data but their morphometric characteristics are shown below:

\begin{tabular}{lccc}
\hline Shape indices & Mean \pm Sd & Minimum & Maximum \\
\hline OL/TL (\%) & $2.12 \pm 0.14$ & 1.95 & 2.22 \\
OH/OL (\%) & $52.22 \pm 7.65$ & 46.46 & 60.90 \\
OT/OL (\%) & $13.36 \pm 1.73$ & 11.81 & 15.22 \\
OT/OH (\%) & $25.62 \pm 0.74$ & 25.00 & 26.44 \\
Circularity & $25.40 \pm 3.69$ & 21.24 & 28.25 \\
Rectangularity & $0.70 \pm 0.01$ & 0.68 & 0.71 \\
\hline
\end{tabular}

\section{FAMILY PERCOPHIDAE}

The rostrum is developed and the antirostrum is frequently absent; the pseudorostrum and pseudoantirostrum are always absent; the ostium is tubular in most cases.

\section{Bembrops heterurus (Miranda Ribeiro 1903) Plate 14}

\begin{tabular}{ll} 
Maximum Size: & $301 \mathrm{~mm}$ (TL) (BERNARDES et al., 2005) \\
$\begin{array}{l}\text { Distribution: } \\
\text { Habitat: }\end{array}$ & Western Atlantic from Rio de Janeiro state, Brazil to Uruguay (BERNARDES et al., 2005) \\
Diet: & Soft bottom of the continental shelf, from 80 to $600 \mathrm{~m}$ depth (BERNARDES et al., 2005; FROESE; PAULY, 2015) \\
Collection: & Crustaceans and fish (BERNARDES et al., 2005) \\
Sample: & 208 otoliths from 107 fish (TL ranging from 65 to $202 \mathrm{~mm})$ \\
\hline
\end{tabular}

Shape: elliptic to trapezoidal (60\%), elliptic to rectangular. Anterior region: peaked. Posterior region: round (42\%), oblique-round (38\%), flattened, oblique to angled. Dorsal edge: sinuate to entire (65\%), lobed to entire. Ventral edge: sinuate to entire (52\%), lobed to entire, lobed to sinuate. Profile: biconvex (71\%), plane-convex. Rostrum and antirostrum orientation: does not apply (77\%), in agreement. Rostrum: developed. Antirostrum: absent (77\%), underdeveloped. Sulcus acusticus: position: median; orientation: horizontal; opening: ostial; morphology: heterosulcoid; colliculum: heteromorphic; ostium: tubular; cauda: round-oval (90\%), tubular straight.

Statistical differences $(p<0.05)$ within some length classes were obtained for posterior region, ventral edge, profile, rostrum and antirostrum orientation, antirostrum development and cauda morphology. Along the otolith growth statistical differences were found for the posterior region and profile.

\begin{tabular}{lccc}
\hline Shape indices & Mean \pm Sd & Minimum & Maximum \\
\hline OL/TL (\%) & $3.12 \pm 0.39$ & 2.44 & 4.09 \\
OH/OL (\%) & $55.99 \pm 2.13$ & 51.61 & 60.16 \\
OT/OL (\%) & $20.36 \pm 1.33$ & 17.58 & 24.44 \\
OT/OH (\%) & $36.41 \pm 2.68$ & 29.41 & 42.21 \\
Circularity & $17.59 \pm 0.84$ & 15.72 & 19.96 \\
Rectangularity & $0.70 \pm 0.02$ & 0.65 & 0.74 \\
\hline
\end{tabular}


Percophis brasiliensis (Quoy \& Gaimard 1825) Plate 15

\begin{tabular}{ll}
\hline Maximum Size: & $650 \mathrm{~mm}$ (TL) (BERNARDES et al., 2005; MENEZES; FIGUEIREDO, 1985) \\
Distribution: & South-Western Atlantic from Rio de Janeiro to Argentina (MENEZES; FIGUEIREDO, 1985) \\
Habitat: & Demersal from 15 to $128 \mathrm{~m}$ depths (BERNARDES et al., 2005) \\
Diet: & Fish, crustaceans, and mollusks (BERNARDES et al., 2005) \\
Collection: & 46 otoliths from 24 fish (TL ranging from 245 to $590 \mathrm{~mm})$ \\
Sample: & 8 left otoliths categorized into $6,20 \mathrm{~mm}$ classes $(280$ to $580 \mathrm{~mm})$ \\
\hline
\end{tabular}

Shape: spindle-shaped to lanceolated. Anterior region: lanceolated. Posterior region: round (50\%), peaked-round, oblique to peaked. Dorsal edge: lobed to sinuate (75\%), lobed. Ventral edge: sinuate to entire (63\%), lobed to entire, entire. Profile: concave-convex. Rostrum and antirostrum orientation: does not apply. Rostrum: developed. Antirostrum: absent. Sulcus acusticus: position: median; orientation: horizontal; opening: ostial; morphology: heterosulcoid; colliculum: heteromorphic; ostium: tubular (63\%), funnel-like; cauda: tubular straight (63\%), tubular slightly curved.

The small number of otoliths examined did not permit the statistical analysis of the data but their morphometric characteristics are shown below:

\begin{tabular}{lccc}
\hline Shape indices & Mean \pm Sd & Minimum & Maximum \\
\hline OL/TL (\%) & $2.33 \pm 0.12$ & 2.19 & 2.49 \\
OH/OL (\%) & $26.63 \pm 2.06$ & 23.69 & 30.00 \\
OT/OL (\%) & $11.60 \pm 1.45$ & 9.99 & 14.03 \\
OT/OH (\%) & $43.45 \pm 2.27$ & 41.33 & 46.77 \\
Circularity & $26.47 \pm 1.82$ & 23.31 & 28.62 \\
Rectangularity & $0.71 \pm 0.01$ & 0.69 & 0.73 \\
\hline
\end{tabular}

\section{FAMILY PINGUIPEDIDAE}

Pseudopercis numida (Miranda Ribeiro 1903) Plate 16

\begin{tabular}{ll}
\hline Maximum Size: & $1200 \mathrm{~mm}$ (TL) (FROESE; PAULY, 2015) \\
Distribution: & $\begin{array}{l}\text { Southwestern Atlantic from Rio de Janeiro to Rio Grande do Sul, Brazil; also found in Nuevo Gulf, Argentina } \\
\text { (MENEZES et al., 2003; VENERUS et al., 2007) }\end{array}$ \\
Habitat: & Deeper waters of the continental shelf (BERNARDES et al., 2005) \\
Diet: & Small fish and crustaceans (MENEZES; FIGUEIREDO, 1985; FROESE; PAULY, 2015; ELÍAS; RAJOY, 1992) \\
Collection: & 22 otoliths from 11 fish (TL ranging from 128 to $620 \mathrm{~mm}$ ) \\
Sample: & 7 left otoliths categorized into 4, $20 \mathrm{~mm}$ classes $(120$ to $460 \mathrm{~mm})$ \\
\hline
\end{tabular}

Shape: lanceolated (86\%), elliptic. Anterior region: lanceolated (86\%), peaked. Posterior region: angled-round (57\%), peaked-round, round. Dorsal edge: entire (43\%), sinuate to entire, lobed to sinuate. Ventral edge: entire (57\%), lobed to sinuate, sinuate to entire. Profile: concave-convex. Rostrum and antirostrum orientation: in agreement (57\%), does not apply. Rostrum: developed. Antirostrum: underdeveloped (57\%), absent. Pseudorostrum and pseudoantirostrum: absent. Sulcus acusticus: position: median; orientation: horizontal; opening: ostial; morphology: heterosulcoid; colliculum: heteromorphic; ostium: funnel-like; cauda: tubular slightly curved (71\%), tubular straight.

The small number of otoliths examined did not permit the statistical analysis of the data but their morphometric characteristics are shown below: 


\begin{tabular}{lccc}
\hline Shape indices & Mean \pm Sd & Minimum & Maximum \\
\hline OL/TL (\%) & $3.77 \pm 0.56$ & 2.85 & 4.25 \\
OH/OL (\%) & $48.56 \pm 2.65$ & 45.72 & 53.42 \\
OT/OL (\%) & $15.78 \pm 2.05$ & 12.87 & 19.05 \\
OT/OH (\%) & $32.64 \pm 4.89$ & 24.08 & 40.00 \\
Circularity & $18.94 \pm 1.77$ & 17.36 & 21.67 \\
Rectangularity & $0.67 \pm 0.01$ & 0.64 & 0.69 \\
\hline
\end{tabular}

\section{FAMILY BLENIIDAE}

\section{Scartella cristata (Linnaeus 1758) Plate 17}

\begin{tabular}{ll}
\hline Maximum Size: & $120 \mathrm{~mm}$ (TL) (FROESE; PAULY, 2015) \\
Distribution: & Western Atlantic from Florida to Santa Catarina state; Southern parts of the Mediterranean (MENEZES; FIGUEI- \\
Habitat: & REDO, 1985; FROESE; PAULY, 2015) \\
Diet: & Invertebrates and algae (FROESE; PAULY, 2015) \\
Collection: & 29 otoliths from 16 fish (TL ranging from 15 to $24 \mathrm{~mm})$ \\
Sample: & 8 left otoliths categorized into $2,20 \mathrm{~mm}$ classes $(15$ to $20 \mathrm{~mm})$ \\
\hline
\end{tabular}

Shape: elliptic (88\%), discoidal. Anterior region: angled-round (50\%), round, oblique-round. Posterior region: round (75\%), angled-round. Dorsal edge: entire. Ventral edge: entire. Profile: biconvex (75\%), plane-convex. Rostrum and antirostrum orientation: does not apply. Rostrum: underdeveloped. Antirostrum: absent. Pseudorostrum and Pseudoantirostrum: absent. Sulcus acusticus: position: supramedian (88\%), median; orientation: horizontal; opening: ostial; morphology: heterosulcoid; colliculum: heteromorphic; ostium: funnel-like; cauda: tubular strongly curved.

The small number of otoliths examined did not allow statistical analysis of the data but their morphometric characteristics are shown below:

\begin{tabular}{lccc}
\hline Shape indices & Mean \pm Sd & Minimum & Maximum \\
\hline OL/TL (\%) & $3.26 \pm 0.48$ & 2.75 & 3.95 \\
OH/OL (\%) & $72.56 \pm 4.63$ & 62.67 & 78.00 \\
OT/OL (\%) & $35.12 \pm 5.94$ & 26.67 & 43.75 \\
OT/OH (\%) & $48.28 \pm 6.81$ & 40.82 & 60.00 \\
Circularity & $13.49 \pm 0.34$ & 13.12 & 13.94 \\
Rectangularity & $0.77 \pm 0.02$ & 0.72 & 0.80 \\
\hline
\end{tabular}

\section{FAMIILY GOBIIDAE}

Otolith shape is squared; rostrum, antirostrum, pseudorostrum and pseudoantirostrum are always absent; sulcus acusticus opening is mesial with the ostium and cauda oval-round or elliptic.

\section{Bathygobius soporator (Valenciennes 1837) Plate 18}

\begin{tabular}{ll}
\hline $\begin{array}{l}\text { Maximum Size: } \\
\text { Distribution: }\end{array}$ & $\begin{array}{l}165 \mathrm{~mm} \text { (TL) (MENEZES; FIGUEIREDO, 1985) } \\
\text { Eastern Atlantic from Senegal to Angola; Western Atlantic from Florida to Rio Grande do Sul state, Brazil; Medi- } \\
\text { terranean sea (MENEZES; FIGUEIREDO, 1985; FROESE; PAULY, 2015) }\end{array}$ \\
Habitat: & Rocky tidal pools and muddy bottom in estuaries (MENEZES; FIGUEIREDO, 1985) \\
Diet: & Zoobenthos (little crabs and shrimps) (FROESE; PAULY, 2015) \\
Collection: & 713 otoliths from 402 fish (TL ranging from 13 to $135 \mathrm{~mm})$ \\
Sample: & 60 left otoliths categorized into 7, $20 \mathrm{~mm}$ classes (13 to $120 \mathrm{~mm})$ \\
\hline
\end{tabular}


Shape: square. Anterior region: notched (85\%), flattened, notched-round, angled. Posterior region: flattened (43\%), oblique, oblique to peaked, round. Dorsal edge: lobed (40\%), sinuate (37\%), lobed to sinuate, entire. Ventral edge: sinuate (70\%), entire, lobed. Anterior edge: sinuate (55\%), entire. Posterior edge: sinuate (77\%), entire, lobed, lobed to sinuate. Profile: concave-convex (83\%), plane-convex. Rostrum and antirostrum orientation: in agreement (87\%), does not apply. Sulcus acusticus: position: median; orientation: descending (88\%), horizontal; opening: mesial; morphology: heterosulcoid; colliculum: heteromorphic; ostium: round-oval; cauda: elliptic.

Statistical differences $(p<0.05)$ within some length classes were obtained for anterior and posterior regions, dorsal, ventral, anterior and posterior edges, profile, rostrum, antirostrum and sulcus acusticus orientation. Along the development, statistical differences were found for posterior region, dorsal, ventral, anterior and posterior edges and profile.

\begin{tabular}{lccc}
\hline Shape indices & Mean \pm Sd & Minimum & Maximum \\
\hline OL/TL (\%) & $3.39 \pm 0.44$ & 2.66 & 4.65 \\
OH/OL (\%) & $88.57 \pm 8.28$ & 73.16 & 108.20 \\
OT/OL (\%) & $22.74 \pm 5.56$ & 16.22 & 44.07 \\
OT/OH (\%) & $25.57 \pm 4.78$ & 18.62 & 41.94 \\
Circularity & $16.57 \pm 2.16$ & 6.24 & 21.91 \\
Rectangularity & $0.78 \pm 0.05$ & 0.72 & 1.05 \\
\hline
\end{tabular}

Ctenogobius smaragdus (Valenciennes 1837) Plate 19

\begin{tabular}{ll}
\hline Maximum Size: & $150 \mathrm{~mm}$ (TL) (FROESE; PAULY, 2015) \\
Distribution: & Western Atlantic, from South Carolina to Southeastern Brazilian coast (MENEZES; FIGUEIREDO, 1985; ME- \\
Habitat: & Shallow waters over muddy bottoms of the continental shelf (FROESE; PAULY, 2015) \\
Diet: & -- \\
Collection: & 1 otolith from 1 fish (TL: $45 \mathrm{~mm})$ \\
Sample: & 1 right otolith $(45 \mathrm{~mm})$ \\
\hline
\end{tabular}

Shape: square. Anterior region: round. Posterior region: blunt-round. Dorsal edge: lobed to entire. Ventral edge: entire. Anterior edge: lobed. Posterior edge: entire. Profile: biconvex. Rostrum and antirostrum orientation: does not apply. Sulcus acusticus: position: median; orientation: ascending; opening: mesial; morphology: heterosulcoid; colliculum: heteromorphic; ostium: elliptic; cauda: round-oval.

Since only one otolith was analyzed it was not possible statistical analysis of the data but its morphometric characteristics are shown below:

\begin{tabular}{lccc}
\hline Shape indices & Mean \pm Sd & Minimum & Maximum \\
\hline OL/TL (\%) & $2.56 \pm 0$ & 2.56 & 2.56 \\
OH/OL (\%) & $106.96 \pm 0$ & 106.96 & 106.96 \\
OT/OL (\%) & $29.57 \pm 0$ & 29.57 & 29.57 \\
OT/OH (\%) & $27.64 \pm 0$ & 27.64 & 27.64 \\
Circularity & $15.95 \pm 0$ & 15.95 & 15.95 \\
Rectangularity & $0.76 \pm 0$ & 0.76 & 0.76 \\
\hline
\end{tabular}


Ctenogobius boleosoma (Jordan \& Gilbert 1882) Plate 20

\begin{tabular}{ll}
\hline Maximum Size: & $75 \mathrm{~mm}$ (TL) (FROESE; PAULY, 2015) \\
Distribution: & $\begin{array}{l}\text { Western Atlantic, from North Carolina, Bahamas, and northern Gulf of Mexico to Southeastern Brazilian coast } \\
\text { (MENEZES; FIGUEIREDO, 1985; MENEZES et al., 2003) }\end{array}$ \\
Habitat: & Found on tide pools and estuaries with cryptic habitats and burrows (WYANSKI; TARGETT, 2000) \\
Diet: & Mainly crustaceans (CORREA; UIEDA, 2007) \\
Collection: & 778 otoliths from 439 fish (TL ranging from 12 to $55 \mathrm{~mm})$ \\
Sample: & 30 left otoliths categorized into 3, $20 \mathrm{~mm}$ classes (14 to $40 \mathrm{~mm}$ ) \\
\hline
\end{tabular}

Shape: square (70\%), discoidal. Anterior region: flattened (47\%), round, blunt, angled-round. Posterior region: bluntround (63\%), round, oblique-round. Dorsal edge: entire (73\%), sinuate to entire, sinuate. Ventral edge: entire (97\%), sinuate to entire. Anterior edge: entire (57\%), does not apply, sinuate to entire. Posterior edge: entire (70\%), does not apply. Profile: plane-convex. Rostrum and antirostrum orientation: does not apply. Sulcus acusticus: position: median; orientation: ascending; opening: mesial; morphology: heterosulcoid; colliculum: heteromorphic; ostium: round-oval; cauda: elliptic $(60 \%)$, round-oval.

Statistical differences $(p<0.05)$ within some length classes were obtained for shape, anterior and posterior regions and dorsal, ventral, anterior and posterior edges. Along the growth statistical differences were found for shape, anterior and posterior regions and dorsal, anterior and posterior edges.

\begin{tabular}{lccc}
\hline Shape indices & Mean \pm Sd & Minimum & Maximum \\
\hline OL/TL (\%) & $3.59 \pm 0.45$ & 2.70 & 4.47 \\
OH/OL (\%) & $97.12 \pm 6.25$ & 84.09 & 108.70 \\
OT/OL (\%) & $31.19 \pm 5.31$ & 24.06 & 39.62 \\
OT/OH (\%) & $31.96 \pm 3.93$ & 26.45 & 39.29 \\
Circularity & $13.40 \pm 2.38$ & 1.15 & 15.01 \\
Rectangularity & $0.79 \pm 0.02$ & 0.73 & 0.82 \\
\hline
\end{tabular}

\section{FAMILY EPHIPPIDAE}

\section{Chaetodipterus faber (Broussonet 1782) Plate 21}

\begin{tabular}{|c|c|}
\hline Maximum Size: & 910 mm (TL) (MENEZES; FIGUEIREDO, 1985) \\
\hline Distribution: & Western Atlantic, from New England to Southern Brazil (MENEZES; FIGUEIREDO, 1985; MENEZES et al., 2003) \\
\hline Habitat: & Coastal waters (estuaries, mangroves and sandy beaches) (FIGUEIREDO et al., 2002) \\
\hline Diet: & Invertebrates (crustaceans, mollusks, cnidarians) (HAYSE, 1990; FIGUEIREDO et al., 2002) \\
\hline Collection: & 60 otoliths from 32 fish (TL ranging from 26 to $283 \mathrm{~mm}$ ) \\
\hline Sample: & 20 left otoliths categorized into $8,20 \mathrm{~mm}$ classes (20 to $280 \mathrm{~mm}$ ) \\
\hline
\end{tabular}

Shape: elliptic (40\%), elliptic to rectangular (35\%), rectangular to fusiform, elliptic to discoidal. Anterior region: peaked-round (50\%), peaked, angled-round. Posterior region: round (75\%), oblique, oblique-round. Dorsal edge: sinuate (40\%), sinuate to entire, lobed to sinuate, crenate to entire. Ventral edge: sinuate to entire (35\%), lobed to sinuate, sinuate, crenate. Profile: concave-convex (65\%), flattened, plane-convex. Rostrum and antirostrum orientation: in agreement (70\%), does not apply. Rostrum: developed (85\%), underdeveloped. Antirostrum: underdeveloped (50\%), developed, absent. Pseudorostrum and Pseudoantirostrum: absent. Sulcus acusticus: position: median; orientation: horizontal; opening: ostial; morphology: heterosulcoid; colliculum: heteromorphic; ostium: funnel-like; cauda: tubular slightly curved $(85 \%)$, round-oval, tubular strongly curved. 
Statistical differences $(p<0.05)$ within some length classes were obtained for shape, anterior and posterior regions, dorsal and ventral edges, cauda morphology, rostrum and antirostrum orientation, profile and rostrum development. Along the development statistical differences were found for shape, anterior region, ventral edge and profile.

\begin{tabular}{lccc}
\hline Shape indices & Mean \pm Sd & Minimum & Maximum \\
\hline OL/TL (\%) & $3.84 \pm 0.67$ & 2.35 & 5.09 \\
OH/OL (\%) & $58.64 \pm 10.94$ & 44.61 & 87.00 \\
OT/OL (\%) & $19.11 \pm 4.2$ & 13.96 & 26.58 \\
OT/OH (\%) & $33.27 \pm 8.94$ & 25.65 & 54.97 \\
Circularity & $19.75 \pm 2.33$ & 16.54 & 25.28 \\
Rectangularity & $0.71 \pm 0.03$ & 0.67 & 0.75 \\
\hline
\end{tabular}

\section{FAMILY SPHYRAENIDAE}

Otolith shape is normally spindle-shaped; the posterior region is oblique or flattened; the profile is concave-convex; the rostrum and antirostrum orientation are in agreement with the rostrum developed and antirostrum frequently underdeveloped; pseudorostrum and pseudoantirostrum are always absent; the ostium is funnel-like and the cauda is predominantly tubular slightly curved.

Sphyraena barracuda (Walbaum 1792) Plate 22

\begin{tabular}{|c|c|}
\hline Maximum Size: & 910 mm (TL) (MENEZES; FIGUEIREDO, 1985) \\
\hline Distribution: & Western Atlantic, from New England to Southern Brazil (MENEZES; FIGUEIREDO, 1985; MENEZES et al., 2003) \\
\hline Habitat: & Coastal waters (estuaries, mangroves and sandy beaches) (FIGUEIREDO et al., 2002) \\
\hline Diet: & Invertebrates (crustaceans, mollusks, cnidarians) (HAYSE, 1990; FIGUEIREDO et al., 2002) \\
\hline Collection: & 6 otoliths from 3 fish (TL ranging from 436 to $563 \mathrm{~mm}$ ) \\
\hline Sample: & 2 left otoliths categorizad into 2, $20 \mathrm{~mm}$ classes (460 to $580 \mathrm{~mm}$ ) \\
\hline
\end{tabular}

Shape: spindle-shaped to lanceolated. Anterior region: lanceolated. Posterior region: oblique. Dorsal edge: lobed to sinuate. Ventral edge: sinuate. Profile: concave-convex. Rostrum and antirostrum orientation: does not apply. Rostrum: developed. Antirostrum: absent. Sulcus acusticus: position: median; orientation: horizontal; opening: ostial; morphology: heterosulcoid; colliculum: heteromorphic; ostium: funnel-like; cauda: tubular slightly curved.

Since only one otolith was analyzed it was not possible statistical analysis of the data but its morphometric characteristics are shown below:

\begin{tabular}{lccc}
\hline Shape indices & Mean \pm Sd & Minimum & Maximum \\
\hline OL/TL (\%) & $2.50 \pm 0$ & 2.50 & 2.91 \\
OH/OL (\%) & $31.25 \pm 0$ & 31.25 & 31.81 \\
OT/OL (\%) & $12.24 \pm 0$ & 12.24 & 13.59 \\
OT/OH (\%) & $39.18 \pm 0$ & 39.18 & 42.73 \\
Circularity & $25.88 \pm 0$ & 21.64 & 25.88 \\
Rectangularity & $0.67 \pm 0$ & 0.67 & 0.73 \\
\hline
\end{tabular}


Sphyraena guachancho Cuvier 1829 Plate 23

\begin{tabular}{ll}
\hline Maximum Size: & $2000 \mathrm{~mm}$ (TL) (CARVALHO-FILHO, 1992) \\
Distribution: & Western Atlantic from Massachusetts to Argentina, also found in the Eastern Atlantic (CARVALHO-FILHO, \\
& 1992; MENEZES et al., 2003) \\
Habitat: & Shallow and coastal waters over muddy bottoms, often found in estuaries (MENEZES; FIGUEIREDO, 1985) \\
Diet: & Mainly fish and crustaceans (MENEZES; FIGUEIREDO, 1985) \\
Collection: & 179 otoliths from 93 fish (TL ranging from 140 to $644 \mathrm{~mm})$ \\
Sample: & 7 left otoliths categorized into 7, $20 \mathrm{~mm}$ classes $(140$ to $440 \mathrm{~mm})$ \\
\hline
\end{tabular}

Shape: spindle-shaped (71\%), rectangular. Anterior region: peaked. Posterior region: oblique (57\%), flattened, round. Dorsal edge: sinuate to entire (71\%), lobed to sinuate. Ventral edge: sinuate to entire (57\%), entire. Profile: concaveconvex. Rostrum and antirostrum orientation: in agreement (86\%), does not apply. Rostrum: developed. Antirostrum: underdeveloped (86\%), absent. Sulcus acusticus: position: median; orientation: horizontal; opening: ostial; morphology: heterosulcoid; colliculum: heteromorphic; ostium: funnel-like; cauda: tubular slightly curved (86\%), tubular straight.

The small number of otoliths examined did not permit the statistical analysis of the data but their morphometric characteristics are shown below:

\begin{tabular}{lccc}
\hline Shape indices & Mean \pm Sd & Minimum & Maximum \\
\hline OL/TL (\%) & $3.06 \pm 0.43$ & 2.42 & 3.71 \\
OH/OL (\%) & $35.67 \pm 5.09$ & 30.34 & 43.16 \\
OT/OL (\%) & $13.15 \pm 2.00$ & 11.21 & 15.94 \\
OT/OH (\%) & $36.84 \pm 1.84$ & 34.45 & 40.38 \\
Circularity & $21.24 \pm 0.48$ & 20.67 & 22.20 \\
Rectangularity & $0.73 \pm 0.01$ & 0.72 & 0.74 \\
\hline
\end{tabular}

Sphyraena tome Fowler 1903 Plate 24

\begin{tabular}{ll}
\hline Maximum Size: & $450 \mathrm{~mm}$ (TL) (CARVALHO-FILHO, 1992) \\
Distribution: & Southwestern Atlantic from Rio de Janeiro to northern Argentina (CARVALHO-FILHO, 1992; MENEZES et al., 2003) \\
Habitat: & Pelagic; shallow and coastal waters (MATSUURA; SUZUKI, 1997) \\
Diet: & Mainly fish (MATSUURA; SUZUKI, 1997) \\
Collection: & 13 otoliths from 7 fish (TL ranging from 291 to $412 \mathrm{~mm})$ \\
Sample: & 2 left otoliths categorized into $1,20 \mathrm{~mm}$ class $(400 \mathrm{~mm})$ \\
\hline
\end{tabular}

Shape: spindle-shaped. Anterior region: peaked. Posterior region: flattened. Dorsal edge: sinuate to entire. Ventral edge: entire. Profile: concave-convex. Rostrum and antirostrum orientation: in agreement. Rostrum: developed. Antirostrum: underdeveloped. Sulcus acusticus: position: median; orientation: horizontal; opening: ostial; morphology: heterosulcoid; colliculum: heteromorphic; ostium: funnel-like; cauda: tubular straight (50\%), tubular slightly curved (50\%).

The small number of otoliths examined did not permit the statistical analysis of the data but their morphometric characteristics are shown below:

\begin{tabular}{lccc}
\hline Shape indices & Mean \pm Sd & Minimum & Maximum \\
\hline OL/TL (\%) & $3.15 \pm 0.02$ & 3.14 & 3.17 \\
OH/OL (\%) & $31.32 \pm 1.94$ & 29.95 & 32.69 \\
OT/OL (\%) & $12.84 \pm 0.20$ & 12.70 & 12.98 \\
OT/OH (\%) & $41.07 \pm 1.91$ & 39.72 & 42.42 \\
Circularity & $22.09 \pm 0.30$ & 21.87 & 22.30 \\
Rectangularity & $0.73 \pm 0.02$ & 0.71 & 0.74 \\
\hline
\end{tabular}




\section{FAMILY GEMPYLIDAE}

Thyrsitops lepidopoides (Cuvier 1832) Plate 25

\begin{tabular}{ll}
\hline Maximum Size: & $400 \mathrm{~mm}$ (TL) (HAIMOVICI et al., 2008) \\
Distribution: & $\begin{array}{l}\text { Southwest Atlantic, from Espirito Santo, Brazil, to Argentina, also found in Chile (MENEZES et al., 2003; HAI- } \\
\text { MOVICI et al., 2008) }\end{array}$ \\
Habitat: & Mesobenthopelagic, inhabits the continental slope (BERNARDES et al., 2005; FROESE; PAULY, 2015) \\
Diet: & Small fish and euphausiids (FIGUEIREDO et al., 2002; BERNARDES et al., 2005) \\
Collection: & 1218 otoliths from 657 fish (TL ranging from 27 to $366 \mathrm{~mm})$ \\
Sample: & 59 left otoliths categorized into $14,20 \mathrm{~mm}$ classes $(20$ to $320 \mathrm{~mm})$ \\
\hline
\end{tabular}

Shape: elliptic to lanceolated (83\%), elliptic, rectangular. Anterior region: lanceolated (83\%), peaked. Posterior region: oblique (58\%), round, flattened. Dorsal edge: lobed (41\%), lobed to sinuate, sinuate, dentate to sinuate. Ventral edge: lobed (51\%), lobed to sinuate, dentate to lobed, sinuate. Profile: concave-convex (85\%), plane-convex. Rostrum and antirostrum orientation: in agreement (86\%), does not apply. Rostrum: developed. Antirostrum: underdeveloped (66\%), developed, absent. Pseudorostrum: absent (88\%), underdeveloped, developed. Pseudoantirostrum: absent (90\%), underdeveloped. Sulcus acusticus: position: median; orientation: horizontal; opening: ostial (54\%), ostio-caudal (46\%); morphology: heterosulcoid; colliculum: heteromorphic; ostium: funnel-like; cauda: tubular slightly curved (39\%), tubular straight, tubular strongly curved, elliptic.

Statistical differences $(p<0.05)$ within some length classes were obtained for shape, anterior and posterior regions, dorsal and ventral edges, profile, rostrum and antirostrum orientation, antirostrum development, pseudorostrum and pseudoantirostrum development sulcus acusticus opening and cauda morphology. Along the development, statistical differences were found for shape, anterior and posterior regions, dorsal and ventral edges, profile, antirostrum development and cauda morphology.

\begin{tabular}{lccc}
\hline Shape indices & Mean \pm Sd & Minimum & Maximum \\
\hline OL/TL (\%) & $2.53 \pm 0.36$ & 1.30 & 4.55 \\
OH/OL (\%) & $50.77 \pm 4.41$ & 45.17 & 69.86 \\
OT/OL (\%) & $10.67 \pm 3.59$ & 6.68 & 21.90 \\
OT/OH (\%) & $20.81 \pm 5.66$ & 13.16 & 38.71 \\
Circularity & $22.63 \pm 3.83$ & 15.56 & 36.15 \\
Rectangularity & $0.65 \pm 0.06$ & 0.41 & 0.86 \\
\hline
\end{tabular}

\section{FAMILY TRICHIURIDAE}

Otoliths are longer than taller being fusiform or spindle-shaped; the anterior region is peaked or lanceolated; pseudorostrum and pseudoantirostrum are always absent.

Benthodesmus elongatus (Clarke 1879) Plate 26

\begin{tabular}{ll}
\hline Maximum Size: & $1000 \mathrm{~mm}$ (TL) (FROESE; PAULY, 2015) \\
Distribution: & $\begin{array}{l}\text { Tropical and subtropical waters of the Southern Hemisphere; South Brazilian coast from Cabo Frio to Argentina } \\
\text { (BERNARDES et al., 2005) }\end{array}$ \\
Habitat: & $\begin{array}{l}\text { Juveniles are mesopelagic and adults benthopelagic living between } 170 \text { and } 950 \mathrm{~m} \text { depth (FIGUEIREDO et al., } \\
\text { 2002; BERNARDES et al., 2005) }\end{array}$ \\
Diet: & Crustaceans, small fish and squids (NAKAMURA; PARIN, 1993; FIGUEIREDO et al., 2002) \\
Collection: & 207 otoliths from 114 fish (TL ranging from 256 to $494 \mathrm{~mm}$ ) \\
Sample: & 61 left otoliths categorized into 13, $20 \mathrm{~mm}$ classes $(240$ to $480 \mathrm{~mm})$ \\
\hline
\end{tabular}


Shape: spindle-shaped. Anterior region: peaked-round (90\%), peaked. Posterior region: flattened (38\%), oblique, blunt, peaked-round. Dorsal edge: sinuate to entire (93\%), lobed to sinuate. Ventral edge: sinuate to entire (84\%), entire, lobed to sinuate. Profile: flattened. Rostrum and antirostrum orientation: does not apply. Rostrum: absent (69\%), developed. Antirostrum: absent. Sulcus acusticus: position: median; orientation: horizontal; opening: mesial (38\%), pseudo-ostial (31\%), ostial (31\%); morphology: archaesulcoid (77\%), pseudo-archaesulcoid; colliculum: unimorphic (77\%), heteromorphic; ostium: absent (77\%), tubular, funnel-like; cauda: absent (77\%), tubular straight.

Statistical differences $(p<0.05)$ within some length classes were obtained for anterior and posterior regions, dorsal and ventral edges, rostrum development and sulcus acusticus, colliculum, ostium and cauda morphology. Along the development, statistical differences were found only for the posterior region.

\begin{tabular}{lccc}
\hline Shape indices & Mean \pm Sd & Minimum & Maximum \\
\hline OL/TL (\%) & $0.75 \pm 0.05$ & 0.50 & 0.92 \\
OH/OL (\%) & $32.71 \pm 1.47$ & 29.53 & 36.75 \\
OT/OL (\%) & $4.14 \pm 1.28$ & 11.69 & 17.47 \\
OT/OH (\%) & $43.28 \pm 4.03$ & 35.40 & 52.94 \\
Circularity & $21.70 \pm 0.83$ & 19.82 & 24.21 \\
Rectangularity & $0.73 \pm 0.02$ & 0.70 & 0.77 \\
\hline
\end{tabular}

Evoxymetopon taeniatus Gill 1863 Plate 27

\begin{tabular}{ll}
\hline Maximum Size: & $2000 \mathrm{~mm}$ (TL) (PARIN; NAKAMURA, 2002) \\
Distribution: & $\begin{array}{l}\text { Western Atlantic, from Bermuda and Bahamas, and the Caribbean sea to the Southeastern-Southern Brazilian } \\
\text { coast; also found in North Pacific (PARIN; NAKAMURA, 2002; BERNARDES et al., 2005) }\end{array}$ \\
Habitat: & Benthopelagic on continental slope, sometimes on shelf areas (PARIN; NAKAMURA, 2002) \\
Diet: & --- \\
Collection: & 38 otoliths from 22 fish (TL ranging from 512 to $1760 \mathrm{~mm})$ \\
Sample: & 10 left otoliths categorized into $10,20 \mathrm{~mm}$ classes $(500$ to $1640 \mathrm{~mm})$ \\
\hline
\end{tabular}

Shape: fusiform to lanceolated. Anterior region: lanceolated. Posterior region: peaked-round. Dorsal edge: sinuate to entire (80\%), lobed to sinuate. Ventral edge: lobed to sinuate (60\%), sinuate to entire, dentate to sinuate. Profile: flattened. Rostrum and antirostrum orientation: in agreement. Rostrum: developed. Antirostrum: developed (80\%), underdeveloped. Sulcus acusticus: position: median; orientation: horizontal; opening: ostial; morphology: heterosulcoid; colliculum: heteromorphic; ostium: funnel-like (80\%), tubular; cauda: tubular straight.

The small number of otoliths examined did not allow statistical analysis of the data but their morphometric characteristics are shown below:

\begin{tabular}{lccc}
\hline Shape indices & Mean \pm Sd & Minimum & Maximum \\
\hline OL/TL (\%) & $0.71 \pm 0.05$ & 0.65 & 0.71 \\
OH/OL (\%) & $34.24 \pm 1.77$ & 32.02 & 37.50 \\
OT/OL (\%) & $12.91 \pm 1.2$ & 11.18 & 14.68 \\
OT/OH (\%) & $37.67 \pm 2.48$ & 33.62 & 41.13 \\
Circularity & $26.45 \pm 2.34$ & 23.04 & 30.81 \\
Rectangularity & $0.66 \pm 0.02$ & 0.63 & 0.69 \\
\hline
\end{tabular}


Lepidopus altifrons Parin \& Collette 1993 Plate 28

\begin{tabular}{ll} 
Maximum Size: & $95 \mathrm{~mm}$ (TL) (FROESE; PAULY, 2015). \\
Distribution: & Pacific, Indian and Atlantic oceans, present along the Brazilian coast (FIGUEIREDO et al., 2002). \\
Habitat: & Inhabits open seas, from 300 to $2,000 \mathrm{~m}$ depth (FROESE; PAULY, 2015). \\
Diet: & Feeds on crustaceans, krill, fish eggs and larvae (SCOTT; SCOTT, 1988). \\
Collection: & 517 otoliths from 288 fish (TL ranging from 223 to $798 \mathrm{~mm})$ \\
Sample: & 89 left otoliths categorized into $13,20 \mathrm{~mm}$ classes (200 to $800 \mathrm{~mm}$ ) \\
\hline
\end{tabular}

Shape: fusiform (76\%), lanceolated, fusiform to lanceolated. Anterior region: peaked (76\%), lanceolated. Posterior region: round (63\%), flattened, oblique, angled-round. Dorsal edge: sinuate to entire (74\%), entire. Ventral edge: lobed to entire (54\%), lobed to sinuate, entire, sinuate to entire. Profile: flattened. Rostrum and antirostrum orientation: in agreement (79\%), does not apply. Rostrum: developed. Antirostrum: underdeveloped (56\%), developed, absent. Sulcus acusticus: position: median; orientation: horizontal; opening: ostial; morphology: heterosulcoid; colliculum: heteromorphic; ostium: funnel-like; cauda: tubular straight.

Statistical differences $(p<0.05)$ within some length classes were obtained for shape, anterior and posterior regions, dorsal and ventral edges, rostrum and antirostrum orientation and antirostrum development. Along the growth development statistical differences were found for shape, anterior and posterior regions.

\begin{tabular}{lccc}
\hline Shape indices & Mean \pm Sd & Minimum & Maximum \\
\hline OL/TL (\%) & $0.86 \pm 0.08$ & 0.67 & 1.14 \\
OH/OL (\%) & $34.68 \pm 1.94$ & 29.01 & 40.16 \\
OT/OL (\%) & $11.53 \pm 1.28$ & 8.80 & 15.60 \\
OT/OH (\%) & $33.28 \pm 3.52$ & 26.70 & 43.80 \\
Circularity & $23.82 \pm 1.83$ & 19.25 & 29.49 \\
Rectangularity & $0.67 \pm 0.02$ & 0.61 & 0.74 \\
\hline
\end{tabular}

Trichiurus lepturus Linnaeus 1758 Plate 29

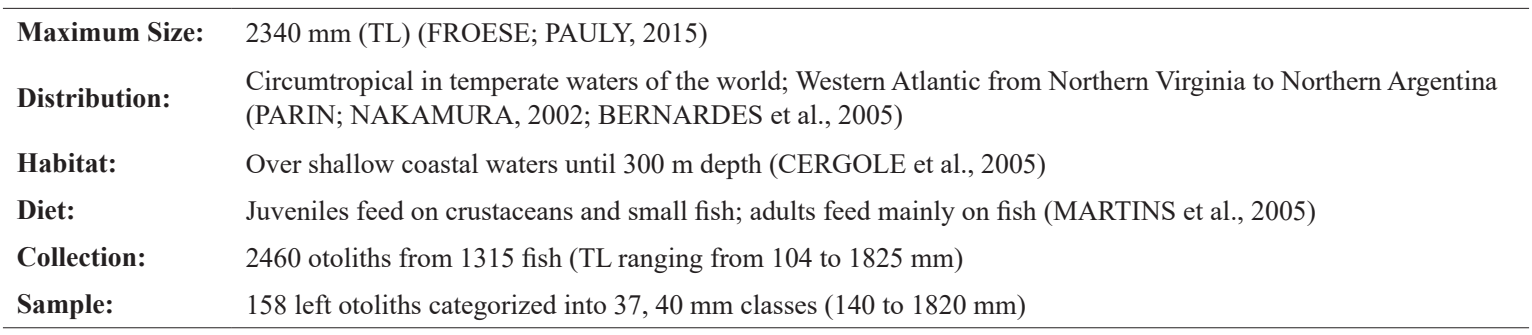

Shape: fusiform. Anterior region: peaked. Posterior region: blunt to peaked (56\%), peaked-round, peaked, angled. Dorsal edge: sinuate (89\%), lobed to sinuate. Ventral edge: sinuate (69\%), lobed to sinuate, lobed, entire. Profile: concave-convex. Rostrum and antirostrum orientation: in agreement (72\%), does not apply. Rostrum: developed. Antirostrum: underdeveloped (61\%), absent, developed. Sulcus acusticus: position: median; orientation: horizontal; opening: ostial; morphology: heterosulcoid; colliculum: heteromorphic; ostium: funnellike (98\%), elliptic; cauda: tubular straight. 
Statistical differences $(p<0.05)$ within some length classes were obtained for posterior region, dorsal and ventral edges, rostrum and antirostrum orientation, antirostrum development, and ostium morphology. Along the growth statistical differences were found only for the posterior region.

\begin{tabular}{lccc}
\hline Shape indices & Mean \pm Sd & Minimum & Maximum \\
\hline OL/TL (\%) & $0.78 \pm 0.26$ & 0.40 & 2.42 \\
OH/OL (\%) & $38.97 \pm 1.99$ & 32.80 & 44.67 \\
OT/OL (\%) & $19.02 \pm 1.65$ & 14.96 & 23.63 \\
OT/OH (\%) & $48.83 \pm 3.65$ & 38.51 & 57.70 \\
Circularity & $20.34 \pm 2.04$ & 0.06 & 28.63 \\
Rectangularity & $0.70 \pm 0.04$ & 0.66 & 0.75 \\
\hline
\end{tabular}

\section{FAMILY SCOMBRIDAE}

Katsuwonus pelamis (Linnaeus 1758) Plate 30

\begin{tabular}{ll}
\hline Maximum Size: & $1100 \mathrm{~mm}$ (TL) (FROESE; PAULY, 2015) \\
Distribution: & Cosmopolitan in tropical and temperate seas; Western Atlantic from Massachusetts to Southern Brazil (COLLET- \\
& TE, 2002; MENEZES et al., 2003) \\
Habitat: & Deep coastal and oceanic waters; forming large schools (CARVALHO-FILHO, 1992; COLLETTE, 2002) \\
Diet: & Fish, crustaceans and cephalopods (TANABE, 2001) \\
Collection: & 9 otoliths from 8 fish (TL ranging from 333 to $630 \mathrm{~mm})$ \\
Sample: & 2 right otoliths categorized into $2,20 \mathrm{~mm}$ classes $(360$ to $460 \mathrm{~mm})$ \\
\hline
\end{tabular}

Shape: rectangular to lanceolated. Anterior region: lanceolated-round. Posterior region: double-peaked-round. Dorsal edge: sinuate to entire. Ventral edge: dentate to lobed. Profile: concave-convex. Rostrum and antirostrum orientation: in agreement. Rostrum: developed. Antirostrum: underdeveloped (50\%), developed (50\%). Pseudorostrum: developed. Pseudoantirostrum: underdeveloped (50\%), developed (50\%). Sulcus acusticus: position: median; orientation: horizontal; opening: ostio-caudal; morphology: heterosulcoid; colliculum: heteromorphic; ostium: funnel-like; cauda: tubular slightly curved.

The small number of otoliths examined did not permit the statistical analysis of the data but their morphometric characteristics are shown below:

\begin{tabular}{lccc}
\hline Shape indices & Mean \pm Sd & Minimum & Maximum \\
\hline OL/TL (\%) & $1.04 \pm 0.02$ & 1.02 & 1.05 \\
OH/OL (\%) & $45.02 \pm 1.8$ & 43.75 & 46.29 \\
OT/OL (\%) & $19.40 \pm 0.91$ & 18.75 & 20.04 \\
OT/OH (\%) & $43.07 \pm 0.31$ & 42.86 & 43.29 \\
Circularity & $38.26 \pm 5.34$ & 34.49 & 42.04 \\
Rectangularity & $0.63 \pm 0.03$ & 0.61 & 0.65 \\
\hline
\end{tabular}




\section{FAMILY ARIOMMATIDAE}

Ariomma bondi Fowler 1930 Plate 31

\begin{tabular}{ll} 
Maximum Size: & $300 \mathrm{~mm}$ (TL) (FROESE; PAULY, 2015) \\
Distribution: & Western Atlantic from Gulf of Maine and the northern Gulf of Mexico to Uruguay; also West Africa (HAEDRI- \\
Habitat: & $\begin{array}{l}\text { Demersal or benthopelagic on the outer continental shelf; juveniles occur in surface waters. (BERNARDES et al., } \\
\text { 2005; HAIMOVICI et al., 2008) }\end{array}$ \\
Diet: & Mainly small crustaceans (HAEDRICH, 2002) \\
Collection: & 1727 otoliths from 994 fish (TL ranging from 15 to $227 \mathrm{~mm})$ \\
Sample: & 88 left otoliths categorized into $10,20 \mathrm{~mm}$ classes $(15$ to $180 \mathrm{~mm})$ \\
\hline
\end{tabular}

Shape: elliptic to lanceolated (72\%), elliptic, elliptic to discoidal. Anterior region: lanceolated (65\%), peaked. Posterior region: round (75\%), flattened, oblique, double-peaked. Dorsal edge: lobed to sinuate (56\%), lobed, entire, sinuate to entire. Ventral edge: lobed to sinuate (58\%), lobed, sinuate to entire. Profile: concave-convex. Rostrum and antirostrum orientation: in agreement (91\%), in disagreement, does not apply. Rostrum: developed. Antirostrum: underdeveloped (70\%), developed, absent. Pseudorostrum and Pseudoantirostrum: absent. Sulcus acusticus: position: median; orientation: ascending (57\%), horizontal; opening: ostial (94\%), ostio-caudal; morphology: heterosulcoid; colliculum: heteromorphic; ostium: funnel-like; cauda: tubular slightly curved (50\%), tubular strongly curved, tubular straight, round-oval.

Statistical differences $(p<0.05)$ within some length classes were obtained for shape, anterior and posterior regions, dorsal and ventral edges, rostrum and antirostrum orientation, antirostrum development, sulcus acusticus orientation and opening and cauda morphology. Along growth development statistical differences were found for shape, anterior and posterior regions, dorsal edge, sulcus acusticus orientation and cauda morphology.

\begin{tabular}{lccc}
\hline Shape indices & Mean \pm Sd & Minimum & Maximum \\
\hline OL/TL (\%) & $2.82 \pm 0.25$ & 2.03 & 3.67 \\
OH/OL (\%) & $58.90 \pm 6.18$ & 44.11 & 84.21 \\
OT/OL (\%) & $9.07 \pm 2.66$ & 4.68 & 18.18 \\
OT/OH (\%) & $15.29 \pm 3.57$ & 8.33 & 24.39 \\
Circularity & $21.08 \pm 3.57$ & 10.45 & 31.01 \\
Rectangularity & $0.67 \pm 0.04$ & 0.58 & 0.94 \\
\hline
\end{tabular}

\section{FAMILY STROMATEIDAE}

\section{Peprilus paru Linnaeus 1758 Plate 32}

\begin{tabular}{|c|c|}
\hline Maximum Size: & $300 \mathrm{~mm}$ (TL) (HAEDRICH, 2002) but attaining $304 \mathrm{~mm}$ in our collection. \\
\hline Distribution: & Western Atlantic from New York to Argentina (BERNARDES et al., 2005) \\
\hline Habitat: & $\begin{array}{l}\text { Coastal and inshore waters over the continental shelf at depths from } 25 \text { to } 70 \text { m; (CARVALHO-FILHO, 1992; } \\
\text { HAEDRICH, 2002) }\end{array}$ \\
\hline Diet: & Jellyfish, small fish, crustaceans and others invertebrates (BERNARDES et al., 2005) \\
\hline Collection: & 471 otoliths from 245 fish (TL ranging from 37 to $304 \mathrm{~mm}$ ) \\
\hline Sample: & 63 left otoliths categorized into $14,20 \mathrm{~mm}$ classes (20 to $300 \mathrm{~mm}$ ) \\
\hline
\end{tabular}


Shape: elliptic (60\%), fusiform, discoidal to elliptic. Anterior region: peaked. Posterior region: angled (33\%), round, oblique, angled-round. Dorsal edge: sinuate to entire (65\%), lobed, lobed to sinuate, dentate to sinuate. Ventral edge: sinuate to entire (49\%), lobed, lobed to sinuate, dentate to sinuate. Profile: concave-convex. Rostrum and antirostrum orientation: in agreement (90\%), in disagreement, does not apply. Rostrum: developed. Antirostrum: underdeveloped (75\%), developed, absent. Pseudorostrum and Pseudoantirostrum: absent. Sulcus acusticus: position: median; orientation: horizontal; opening: ostial; morphology: heterosulcoid; colliculum: heteromorphic; ostium: funnel-like; cauda: tubular slightly curved (62\%), tubular straight.

Statistical differences $(p<0.05)$ within some length classes were obtained for shape, posterior region, dorsal and ventral edges, rostrum and antirostrum orientation, antirostrum development and cauda morphology. Along the development statistical differences were found for shape, posterior region, dorsal and ventral edges.

\begin{tabular}{lccc}
\hline Shape indices & Mean \pm Sd & Minimum & Maximum \\
\hline OL/TL (\%) & $4.00 \pm 0.60$ & 2.92 & 5.85 \\
OH/OL (\%) & $56.35 \pm 8.71$ & 45.41 & 78.40 \\
OT/OL (\%) & $10.20 \pm 2.17$ & 7.98 & 18.41 \\
OT/OH (\%) & $18.00 \pm 1.40$ & 14.79 & 23.57 \\
Circularity & $21.59 \pm 2.62$ & 17.47 & 31.24 \\
Rectangularity & $0.67 \pm 0.02$ & 0.64 & 0.71 \\
\hline
\end{tabular}

\section{FAMIILY CAPROIDAE}

Antigonia capros Lowe 1843 Plate 33

\begin{tabular}{|c|c|}
\hline Maximum Size: & 305 mm (TL) (FROESE; PAULY, 2015) \\
\hline Distribution: & Circumtropical; Western Atlantic from New Jersey to Uruguay (FIGUEIREDO et al., 2002; BERNARDES, 2005) \\
\hline Habitat: & Adults are found close to bottom and juveniles occur in mid water (FIGUEIREDO et al., 2002) \\
\hline Diet: & Mainly crustaceans, cephalopods and mollusks (BERNARDES et al., 2005) \\
\hline Collection: & 1157 otoliths from 598 fish (TL ranging from 32 to $209 \mathrm{~mm}$ ) \\
\hline Sample: & 73 left otoliths categorized into $9,20 \mathrm{~mm}$ classes (20 to $180 \mathrm{~mm}$ ) \\
\hline
\end{tabular}

Shape: rhomboidal (88\%), tall. Anterior region: double-peaked (60\%), double-peaked-round, peaked, angled. Posterior region: angled-round (67\%), round. Dorsal edge: sinuate to entire (51\%), lobed to sinuate, dentate to sinuate, entire. Ventral edge: sinuate to entire (81\%), lobed to sinuate, serrate to sinuate, entire. Profile: plane-convex. Rostrum and antirostrum orientation: in agreement (97\%), does not apply. Rostrum: developed (85\%), underdeveloped. Antirostrum: underdeveloped (51\%), developed (47\%), absent. Pseudorostrum and Pseudoantirostrum: absent. Sulcus acusticus: position: supramedian; orientation: ascending; opening: ostial (86\%), ostio-caudal; morphology: heterosulcoid; colliculum: heteromorphic; ostium: bent; cauda: tubular straight (84\%), tubular strongly curved, tubular slightly curved.

Statistical differences $(p<0.05)$ within some length classes were obtained for shape, anterior and posterior regions, dorsal and ventral edges, rostrum and antirostrum orientation and development, sulcus acusticus opening and cauda morphology. Along the growth statistical differences were found for shape, anterior region, dorsal edge, antirostrum development and cauda morphology.

\begin{tabular}{lccc}
\hline Shape indices & Mean \pm Sd & Minimum & Maximum \\
\hline OL/TL (\%) & $5.38 \pm 0.79$ & 3.97 & 7.72 \\
OH/OL (\%) & $117.97 \pm 13.13$ & 81.88 & 149.47 \\
OT/OL (\%) & $22.84 \pm 3.94$ & 14.80 & 31.95 \\
OT/OH (\%) & $19.34 \pm 2.29$ & 14.39 & 25.84 \\
Circularity & $18.06 \pm 1.92$ & 14.37 & 23.02 \\
Rectangularity & $0.66 \pm 0.04$ & 0.62 & 0.86 \\
\hline
\end{tabular}




\section{REFERENCES}

BERNARDES, R. A.; FIGUEIREDO, J. L.; RODRIGUES, A. R.; FISCHER, L. G.; VOOREN, C. M.; HAIMOVICI, M.; ROSSI-WONGTSCHOWSKI, C. L. D. B. Peixes da Zona Exclusiva da região Sudeste-Sul do Brasil: levantamento com armadilhas, pargueiras e rede de arrasto de fundo. São Paulo: EDUSP, 2005. 304 p.

BRENHA-NUNES, M. R.; SANTIFICETUR, C.; CONVERSANI, V. R. M.; GIARETTA, M. B.; ROSSI-WONGTSCHOWSKI, C. L. D. B.; SILIPRANDI, C. C. Atlas of marine bony fish otoliths (sagittae) of Southeastern-southern Brazil. Part IV: Perciformes (Centropomidae, Acropomatidae, Serranidae, Priacanthidae, Malacanthidae, Pomatomidae, Carangidae, Lutjanidae, Gerreidae and Haemulidae). Braz. J. Oceanogr., v. 64, n. spe1, p. 23-75, 2016.

BURGESS, W. E. Chaetodontidae, Butterflyfish. In: CARPENTER, K. E. (Ed.). The living marine resources of the Western Central Atlantic. Volume 3: Bony fish part 2 (Opistognathidae to Molidae), sea turtles and marine mammals. FAO Species Identification Guide for Fishery Purposes and American Society of Ichthyologists and Herpetologists Special Publication n ${ }^{\circ}$. 5. Rome: FAO, 2002. p. $1663-1672$.

CARPENTER, K. E. Sparidae, Porgies. In: CARPENTER, K. E. (Ed.). The living marine resources of the Western Central Atlantic. Volume 3: Bony fish part 2 (Opistognathidae to Molidae), sea turtles and marine mammals. FAO Species Identification Guide for Fishery Purposes and American Society of Ichthyologists and Herpetologists Special Publication n ${ }^{\circ}$. 5. Rome: FAO, 2002. p. $1554-1577$.

CARVALHO-FILHO, A. Peixes: costa brasileira. 2. ed. São Paulo: Marca D’Água, 1992. 304 p.

CERGOle, M. C.; ÁVILA-DA-SILVA, A. O.; ROSSI-WONGTSCHOWSKI, C. L. D. B. Análise das Principais Pescarias Comerciais da Região Sudeste-Sul do Brasil: Dinâmica Populacional das Espécies em Explotação. (Série documentos Revizee: Score Sul). São Paulo: Instituto Oceanográfico USP, 2005. $176 \mathrm{p}$.

CERVIGÓN, F.; CIPRIANI, R.; FISCHER, W.; GARIBALDI, L.; HENDRICKX, M.; LEMUS, A. J.; MÁRQUEZ, R.; POUTIERS, J. M.; ROBAINA, G.; RODRIQUEZ, B. Guia de campo de las espécies comerciales marinas y de aguas salobres de la costa septentrional de Sur América. Rome: FAO, 1992. 513 p.

CHAO, N. L. Scianidae, Croakers (drums). In: CARPENTER, K. E. (Ed.). The living marine resources of the Western Central Atlantic. Volume 3: Bony fish part 2 (Opistognathidae to Molidae), sea turtles and marine mammals. FAO Species Identification Guide for Fishery Purposes and American Society of Ichthyologists and Herpetologists Special Publication n ${ }^{\circ}$. 5. Rome: FAO, 2002. p. $1583-1653$.

COLLETTE, B. B. Scombridae, Mackerels and tunas. In: CARPENTER, K. E. (Ed.). The living marine resources of the Western Central Atlantic. Volume 3: Bony fish part 2 (Opistognathidae to Molidae), sea turtles and marine mammals. FAO Species Identification Guide for Fishery Purposes and American Society of Ichthyologists and Herpetologists Special Publication n . 5. Rome: FAO, 2002. p. 1836-1857.

CORREAA, M. O. D. A.; UIEDA, V. S. Diet of the ichthyofauna associated with marginal vegetation of a mangrove forest in southeastern Brazil. Iheringia, Sér. Zool., Porto Alegre, v. 97, n. 4, p. 486-497, 2007.

ELÍAS, I.; RAJOY, C. R. Habitos alimentares del "salmon del mar" Pseudopercis semifasciata (Cuvier, 1829), Pinguipedidae em águas norpatogonicas argentinas. Rev. Bio. Mar. v. 27, n. 1, p. 133-146, 1992.

FELTES, R. M. Polynemidae, Threadfins. In: CARPENTER, K. E. (Ed.). The living marine resources of the Western Central Atlantic. Volume 3: Bony fish part 2 (Opistognathidae to Molidae), sea turtles and marine mammals. FAO Species Identification Guide for Fishery Purposes and American Society of Ichthyologists and Herpetologists Special Publication n ${ }^{\circ}$. 5. Rome: FAO, 2002. p. 1578-1582.

FIGUEIREDO, J. L.; SANTOS, A. P.; YAMAGUTI, N.; BERNARDES, R. A.; ROSSI-WONGTSCHOWSKI, C. L. D. B. Peixes da zona econômica exclusiva da região Sudeste-Sul do Brasil: levantamento com rede de meia-água. São Paulo: EDUSP, 2002.242 p.

FROESE, R.; PAULY, D. FishBase. World Wide Web electronic publication. 2015. Available: < http://www.fishbase.org> Version: 05/2015.

HAEDRICH, R. L. Ariommatidae. In: CARPENTER, K. E. (Ed.). The living marine resources of the Western Central Atlantic. Volume 3: Bony fish part 2 (Opistognathidae to Molidae), sea turtles and marine mammals. FAO Species Identification Guide for Fishery Purposes and American Society of Ichthyologists and Herpetologists Special Publication n . 5. Rome: FAO, 2002. p. 1873-1877.

HAIMOVICI, M.; ROSSI-WONGTSCHOWSKI, C. L. D. B.; BERNARDES, R. A.; FISCHER, L. G.; VOOREN, C. M.; SANTOS, R. A.; RODRIGUES, A. R.; SANTOS, S. Prospecção pesqueira de espécies demersais com rede de arrasto-de-fundo na Região Sudeste-Sul do Brasil. (Série documentos Revizee: Score Sul). São Paulo: Instituto Oceanográfico USP, 2008. 183 p.

HARRISON, I. J. Order Mugiliformes, Mugilidae, Mullets. In: CARPENTER, K. E. (Ed.). The living marine resources of the Western Central Atlantic. Volume 2: Bony fish part 1 (Acipenseridae to Grammatidae). FAO Species Identification Guide for Fishery Purposes and American Society of Ichthyologists and Herpetologists Special Publication n ${ }^{\circ}$. 5. Rome: FAO, 2002. p. 1071-1085.

HAYSE, J. W. Feeding Habits, Age, Growth, and Reproduction of Atlantic Spadefish Cheatodipterus faber (Pisces: Ephippidae) in South Carolina. Fish. Bull., v. 88, n. 1, p. 67-83, 1990.

LABROPOULOU, M.; Machias A.; Tsimenides, N. Habitat selection and diet of juvenile red porgy, Pagrus pagrus (Linnaeus, 1758). Fish. Bull., 97, n. 3, p. 495-507, 1999.

LOPES, P. R. D.; OLIVEIRA-SILVA, J. T. Nota sobre a alimentação de Conodon nobilis (Linnaeus) e Polydactylus virginicus (Linnaeus) (Actinopterygil: Haemulidae e Polynemidae) na praia de Jaguaribe (Ilha de Itamaracá), estado de Pernambuco. Bioikos, v. 12, n. 2, p. 53-59, 1998. 
MAGRO, M.; CERGOLE, M. C.; ROSSI-WONGTSCHOWSKI, C. L. D. B. Síntese de conhecimentos dos principais recursos pesqueiros costeiros potencialmente explotáveis na costa sudeste-sul do Brasil: peixes. Brasília: Ministério do Meio Ambiente, dos Recursos Hídricos e da Amazônia Legal, 2000. 109 p.

MARTINS, A. S.; HAIMOVICI, M.; PALACIOS, R. Diet and feeding of the cutlassfish Trichiurus lepturus in the Subtropical Convergence Ecosystem of southern Brazil. J. Mar. Biol. Ass. UK., v. 85, p. 1223-1229, 2005.

MATSUURA, Y.; SUZUKI, K. Larval development of two species of barracuda, Sphyraena guachancho and S. tome (Teleostei: Sphyraenidae), from southeastern Brazil. Ichthyol. Res., v. 44, n. 4, 369-378, 1997.

MENEZES, N. A.; BUCKUP, P. A.; FIGUEIREDO, J. L.; MOURA, R. L. (Eds.). Catálogo das espécies de peixes marinhos do Brasil. São Paulo: Museu de Zoologia da Universidade de São Paulo, 2003. 160 p.

MENEZES, N. A.; FIGUEIREDO, J. L. Manual de peixes marinhos do Sudeste do Brasil. V. Teleostei (4). São Paulo: Museu de Zoologia da Universidade de São Paulo, 1985. 105 p.

MENEZES, N. A.; FIGUEIREDO, J. L. Manual de peixes marinhos do Sudeste do Brasil. IV. Teleostei (3). São Paulo: Museu de Zoologia da Universidade de São Paulo, 1980. 96 p.

NAKAMURA, I.; PARIN, N. V. FAO Species Catalogue. Vol. 15: Snake Mackerels and Cutlassfish of the World (Families Gempylidae and Trichiuridae). Rome: FAO, 1993.

PARIN, N. V.; NAKAMURA, I. Trichiuridae, Scabbard.fish (hairtails, frostfish). In: CARPENTER, K. E. (Ed.). The living marine resources of the Western Central Atlantic. Volume 3: Bony fish part 2 (Opistognathidae to Molidae), sea turtles and marine mammals. FAO Species Identification Guide for Fishery Purposes and American Society of Ichthyologists and Herpetologists Special Publication $n^{\circ}$. 5. Rome: FAO, 2002, p. 1825-1835.

RANDALL, J. E. Food habitats of reef fish of the West Indies. Stud. Trop. Oceanogr., v. 5, p. 665-847, 1967.

ROSSI-WONGTSCHOWSKI, C. L. D. B.; CHALOM, A.; SILIPRANDI, C. C.; BRENHA-NUNES, M. R.; CONVERSANI, V. R. M.; SANTIFICETUR, C.; GIARETTA, M. B. COSS-Brasil: Coleção de Otólitos de Peixes Marinhos da Região Sudeste-Sul do Brasil. São Paulo: Instituto Oceanográfico da Universidade de São Paulo, 2016.

ROSSI-WONGTSCHOWSKI, C. L. D. B.; SILIPRANDI, C. C.; BRENHA, M. R.; GONSALES, S. A.; SANTIFICETUR, C.; VAZ-DOS-SANTOS, A. M. Atlas of marine bony fish otoliths (sagittae) of Southeastern-southern Brazil. Part I: Gadiformes (Macrouridae, Moridae, Bregmacerotidae, Phycidae and Merlucciidae); Part II: Perciformes (Carangidae, Sciaenidae, Scombridae and Serranidae). Braz. J. Oceanogr., v. 62, n. spe1, p. 1-103, 2014.

SCOTT, W. B.; SCOTT, M. G. Atlantic fishes of Canada. Can. Bull. Fish. Aquat. Sci., v. 3, p. 219-731, 1988.

SILIPRANDI, C. C.; BRENHA-NUNES, M. R.; ROSSI-WONGTSCHOWSKI, C. L. D. B.; SANTIFICETUR, C.; CONVERSANI, V. R. M. Atlas of marine bony fish otoliths (sagittae) of Southeastern-southern Brazil. Part III: Clupeiformes (Clupeidae, Engraulidae, Pristigasteridae). Braz. J. Oceanogr., v. 64, n. spe1, p. 11-28, 2016.

SILVANO, R. A. M.; GÜTH, A. Z. Diet and feeding behavior of Kyphosus spp. (Kyphosidae) in a Brazilian Subtropical Reef. Braz. Arch. Biol. Technol., v. 49, n. 4, p. 623-629, 2006.

TANABE, T. Feeding habits of skipjack tuna Katsuwonus pelamis and other tuna Thunnus spp. juveniles in the tropical western Pacific. Fish. Sci., v. 67, n. 4, p. 563-570, 2001.

VAUGHAN, F. A. Food Habits of the Sea Bream, Archosargus rhomboidalis (Linnaeus), and Comparative Growth on Plant and Animal Food. Bull. Mar. Sci., v. 28, n. 3, p. 527-536, 1978.

VENERUS, L. A.; GALVÁN, D. E.; IRIGOYEN, A. J.; GOSZTONYI, A. E. First record of the namorado sandperch, Pseudopercis numida Miranda-Ribeiro, 1903 (Pinguipedidae; Osteichthyes), in Argentine waters. J. Appl. Ichthyol. v. 23, n. 1, p. 110-112, 2007.

WESTNEAT, M. W. Scaridae, Parrotfish. In: CARPENTER, K. E. (Ed.). The living marine resources of the Western Central Atlantic. Volume 3: Bony fish part 2 (Opistognathidae to Molidae), sea turtles and marine mammals. FAO Species Identification Guide for Fishery Purposes and American Society of Ichthyologists and Herpetologists Special Publication n . 5. Rome: FAO, 2002. p. 1723-1739.

WYANSKI, D. M.; TARGETT, T. E. Development of transformation larvae and juveniles of Ctenogobius boleosoma, Ctenogobius shufeldti, and Gobionellus oceanicus (pisces: gobiidae) from Western North Atlantic estuaries, with notes on early life history. Bull. Mar. Sci., v. 67, n. 2, 709-728, 2000. 
A1

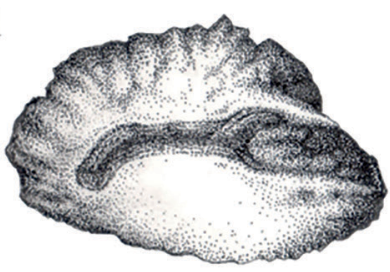

A2

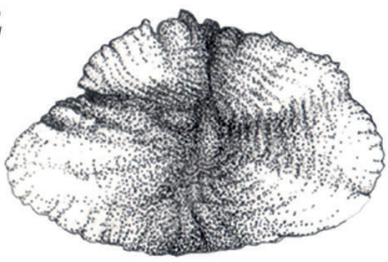

A3

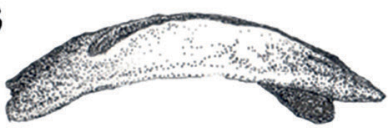

B1

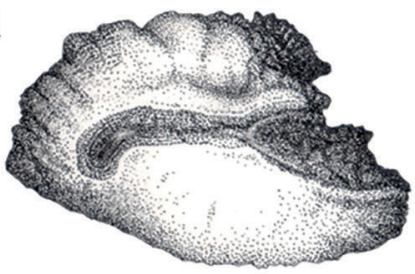

B2

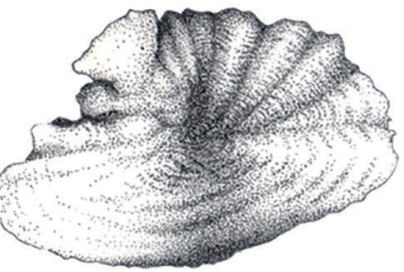

B3

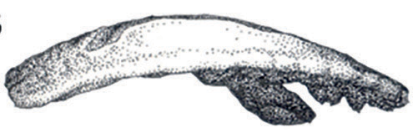

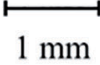

\section{$1 \mathrm{~mm}$}

A1

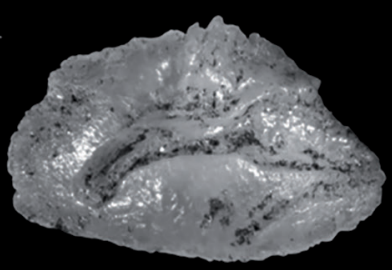

$\mathbf{A 2}$

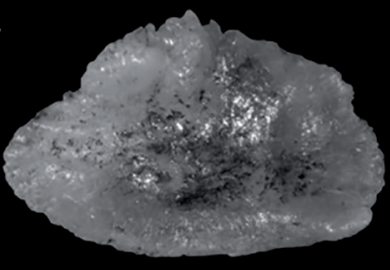

A3

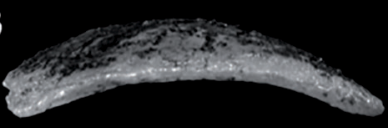

B1

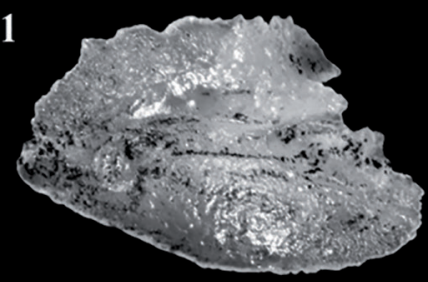

B2

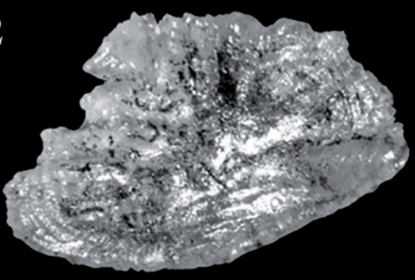

B3

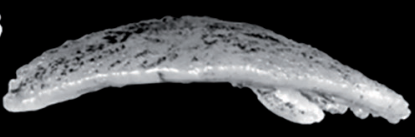

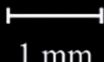

Plate 1. Illustrations (above) and photos (below) of Archosargus rhomboidalis otoliths from fish with total lengths: A. $115 \mathrm{~mm}$; B. $160 \mathrm{~mm}$. The medial face is shown in A1, B1; the lateral face in A2, B2; and the ventral profile in A3, B3 (Illustrations and Photos: Alexandre Arackawa). 

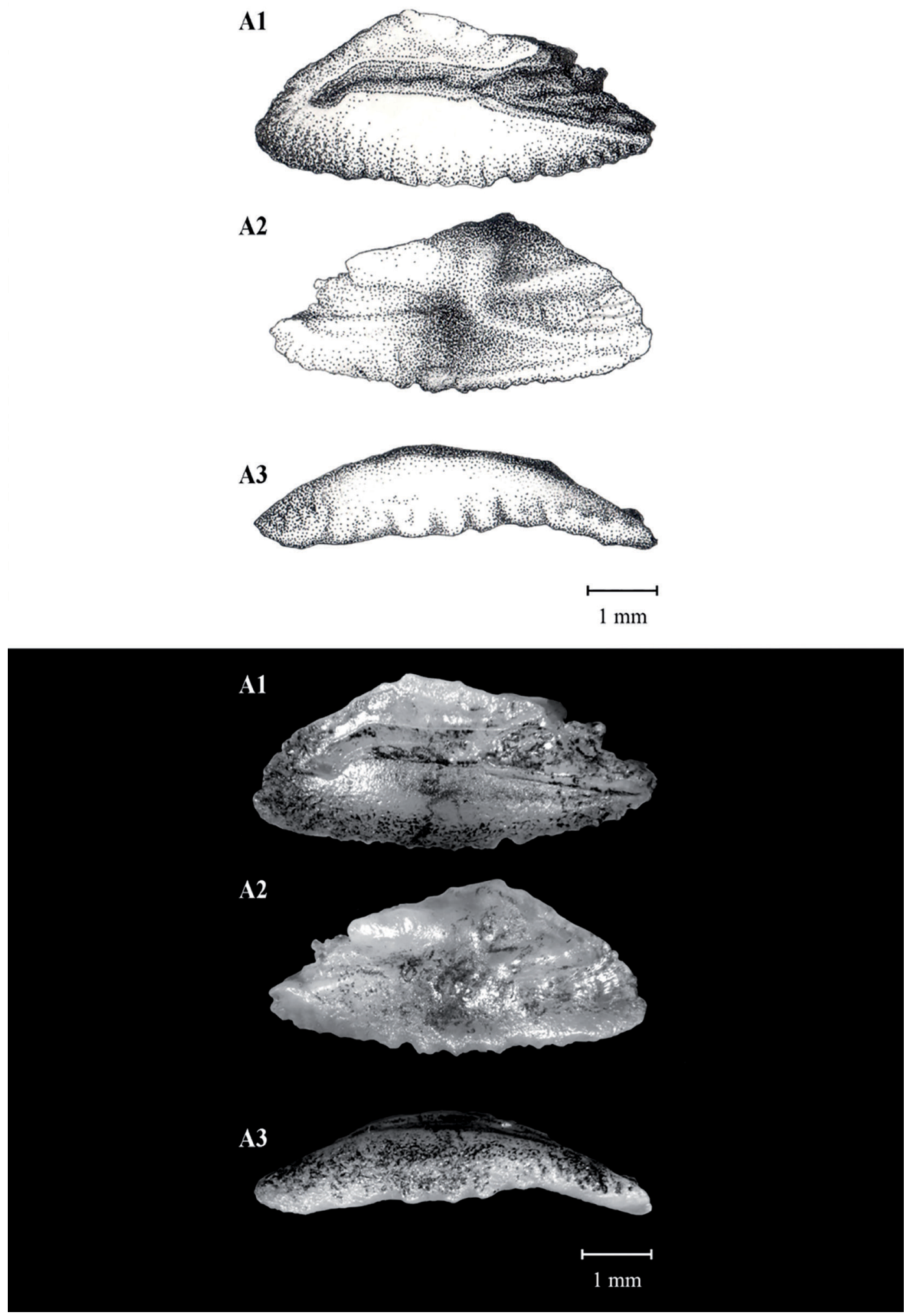

Plate 2. Illustrations (above) and photos (below) of Calamus penna otolith from a fish with $167 \mathrm{~mm}$ total length. The medial face is shown in A1; the lateral face in A2; and the ventral profile in A3 (Illustrations and Photos: Alexandre Arackawa). 
A1

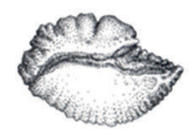

A2

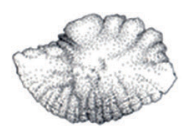

A3

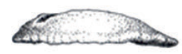

B1

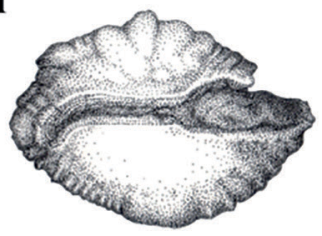

B2

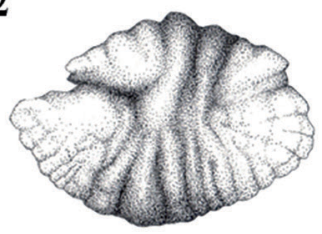

B3

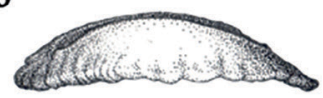

C1

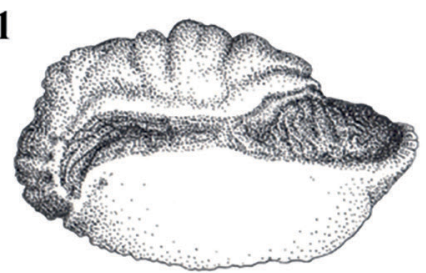

C2

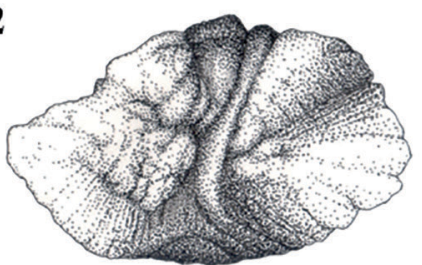

C3

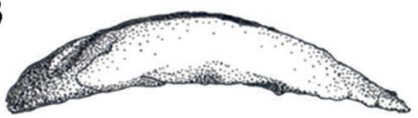

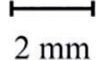

$2 \mathrm{~mm}$
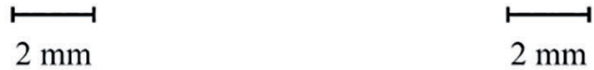

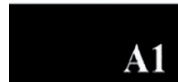

A1

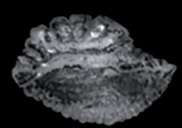

A2

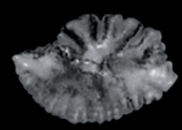

A3

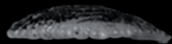

B1

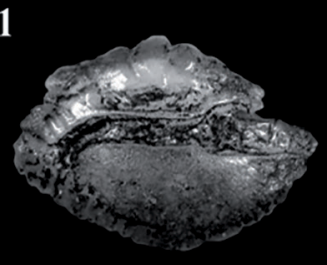

B2

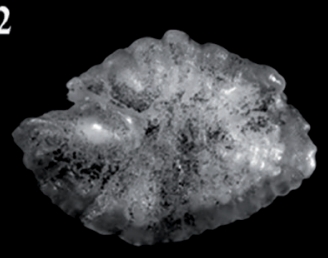

C1

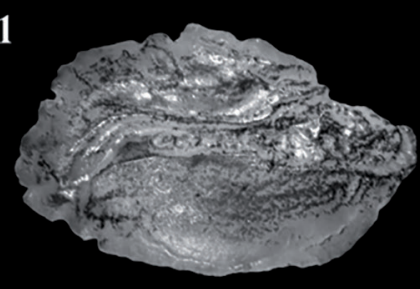

C2

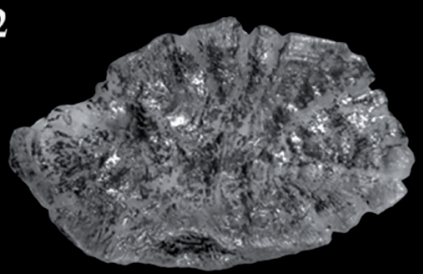

B3

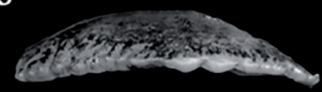

C3

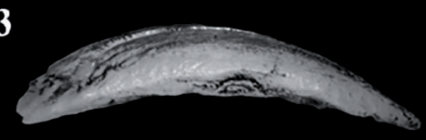

Plate 3. Illustrations (above) and photos (below) of Pagrus pagrus otoliths from fish with total lengths: A. $69 \mathrm{~mm}$; B. $153 \mathrm{~mm}$; C. $273 \mathrm{~mm}$. The medial face is shown in A1, B1, C1; the lateral face in A2, B2, C2; and the ventral profile in A3, B3, C3 (Illustrations: Alexandre Arackawa; Photos: Alexandre 
A1

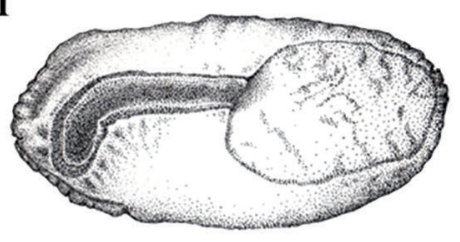

A2

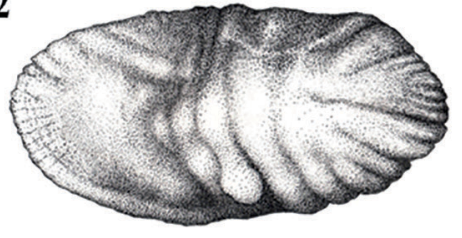

A3

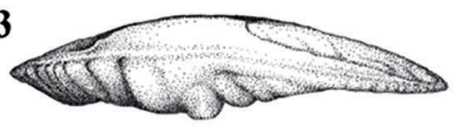

B1

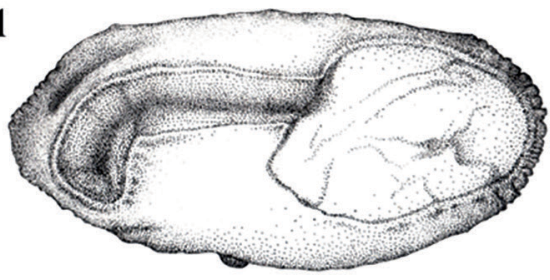

B2

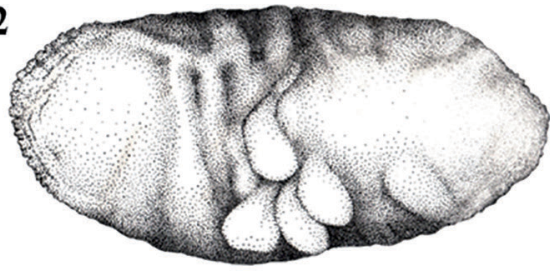

B3

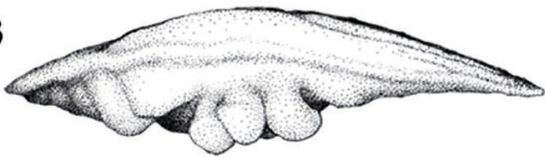

$\longmapsto$

A1

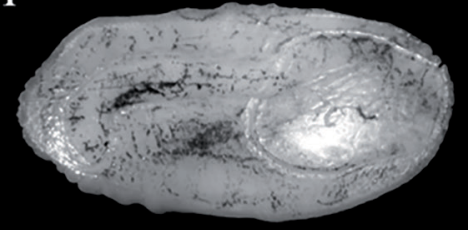

A2

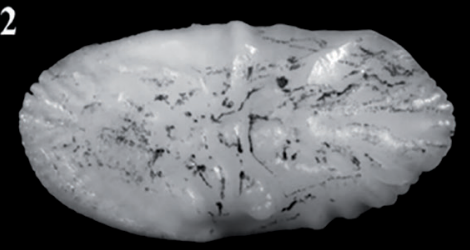

A3

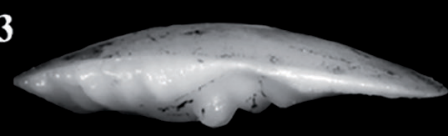

B1

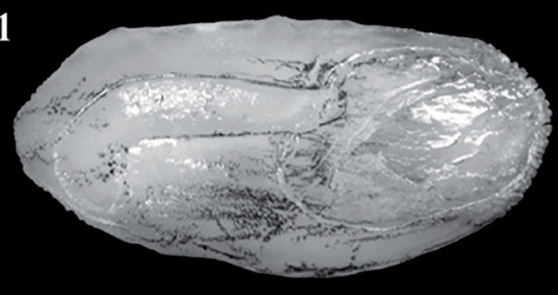

B2

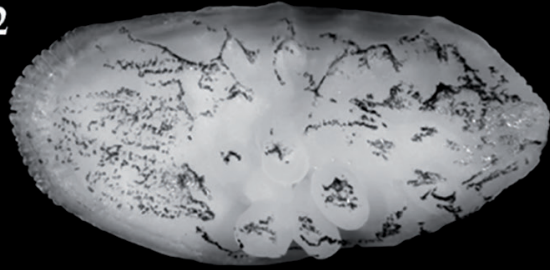

Plate 4. Illustrations (above) and photos (below) of Cynoscion leiarchus otoliths from fish with total lengths: A. 229 mm; B. $292 \mathrm{~mm}$. The medial face is shown in A1, B1; the lateral face in A2, B2; and the ventral profile in A3, B3 (Illustrations and Photos: Alexandre Arackawa). 
A1

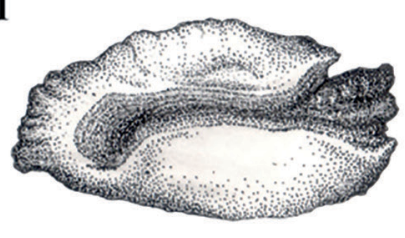

A2

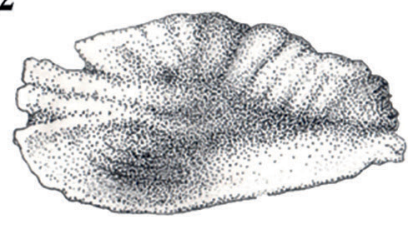

A3

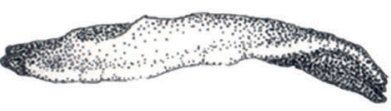

B1

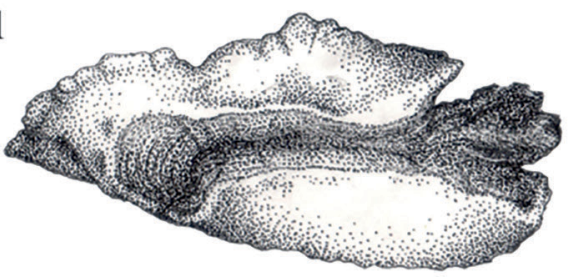

B2

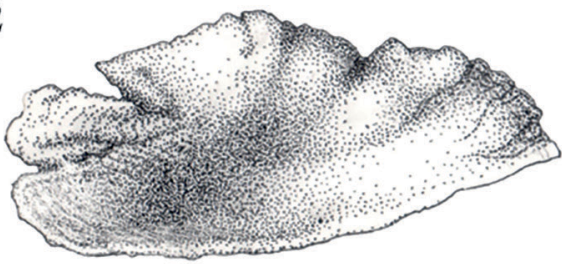

B3

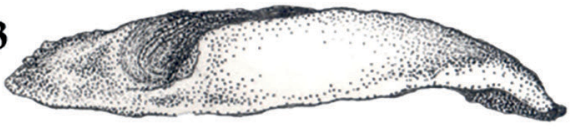

\section{$2 \mathrm{~mm}$}

\section{$2 \mathrm{~mm}$}

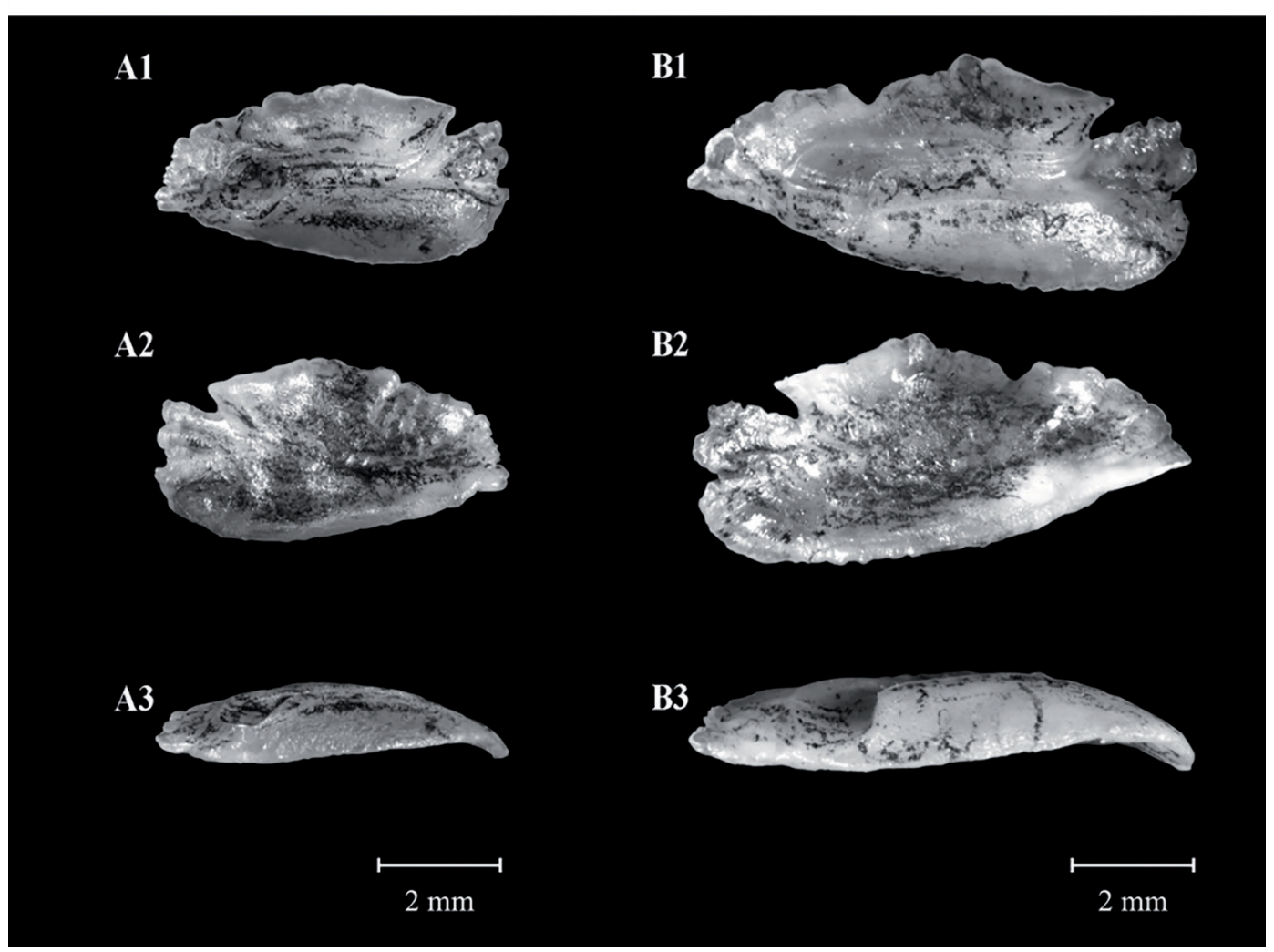

Plate 5. Illustrations (above) and photos (below) of Polydactylus virginicus otoliths from fish with total lengths: A. $215 \mathrm{~mm}$; B. $344 \mathrm{~mm}$. The medial face is shown in A1, B1; the lateral face in A2, B2; and the ventral profile in A3, B3 (Illustrations and Photos: Alexandre Arackawa). 
A1

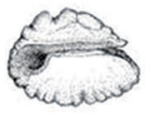

A2

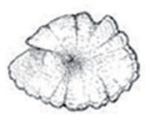

A3

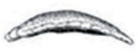

$\longmapsto$

A1

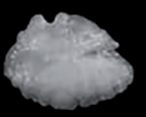

A2

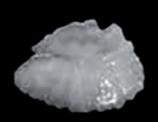

A3

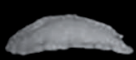

$\longmapsto 1 \mathrm{~mm}$
B1

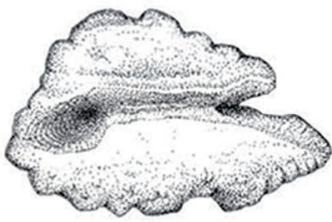

B2

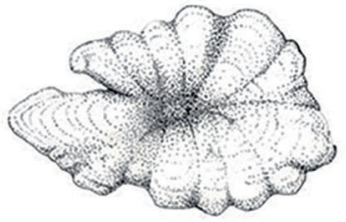

B3

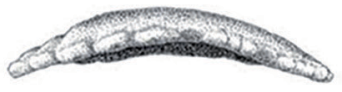

C1

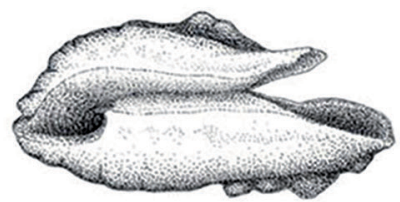

C2

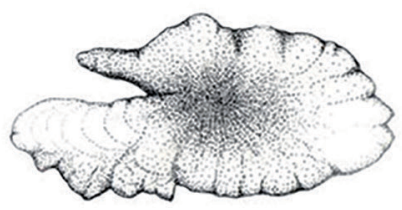

C3

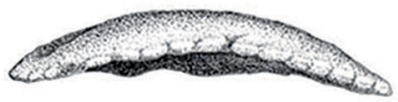

B1

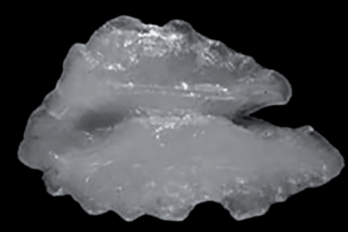

B2

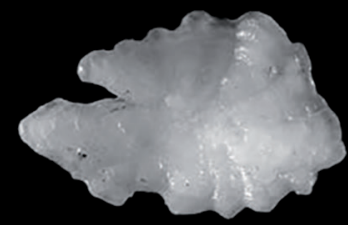

B3

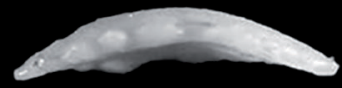

C1

$\mathrm{C} 2$

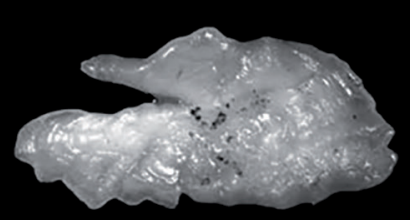

C3

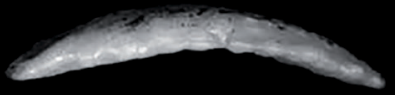

$\longmapsto$

Plate 6. Illustrations (above) and photos (below) of Mullus argentinae otoliths from fish with total lengths: A. $129 \mathrm{~mm} ; \mathrm{B} .192 \mathrm{~mm}$; C. $243 \mathrm{~mm}$. The medial face is shown in A1, B1, C1; the lateral face in A2, B2, C2; and the ventral profile in A3, B3, C3 (Illustrations: Laura Montserrat; Photos: Cesar Santificetur). 
A1

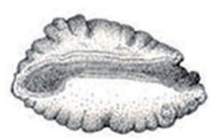

A2

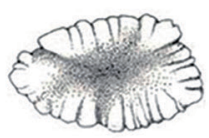

A3

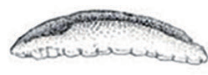

$2 \mathrm{~mm}$
B1

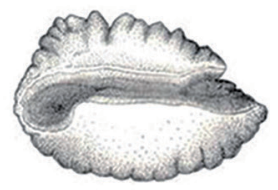

B2

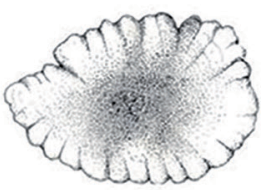

B3
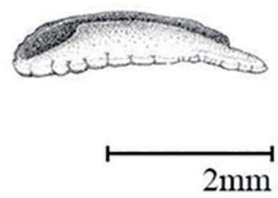

C1

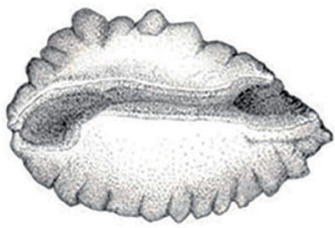

C2

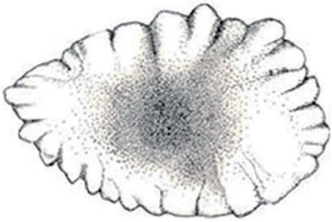

C3

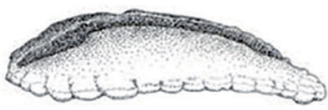

$2 \mathrm{~mm}$
A1

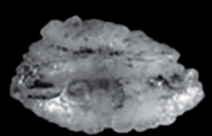

A2

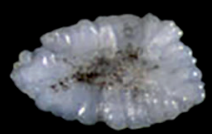

A3
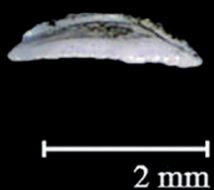

B1

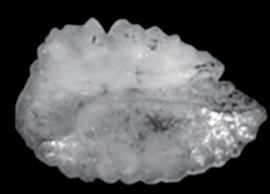

B2

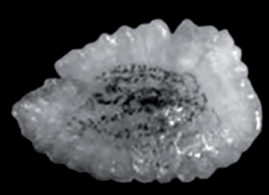

B3
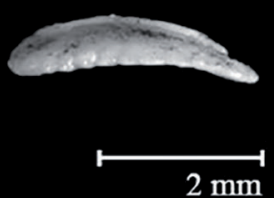

C1

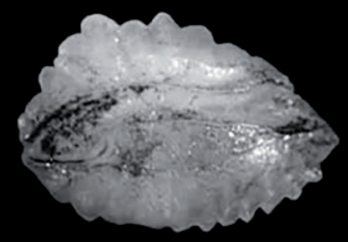

C2

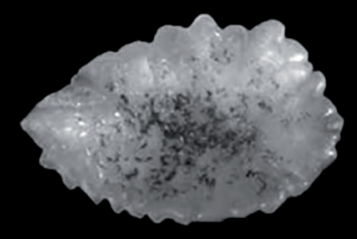

C3

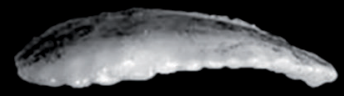

$2 \mathrm{~mm}$

Plate 7. Illustrations (above) and photos (below) of Upeneus parvus otoliths from fish with total lengths: A. $84 \mathrm{~mm}$; B. $129 \mathrm{~mm}$; C. $175 \mathrm{~mm}$. The medial face is shown in A1, B1, C1; the lateral face in A2, B2, C2; and the ventral profile in A3, B3, C3 (Illustrations: Laura Montserrat; Photos: Cesar Santificetur). 


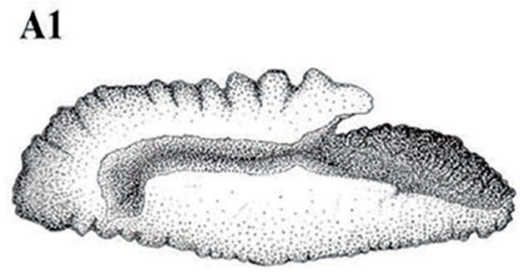

\section{A2}
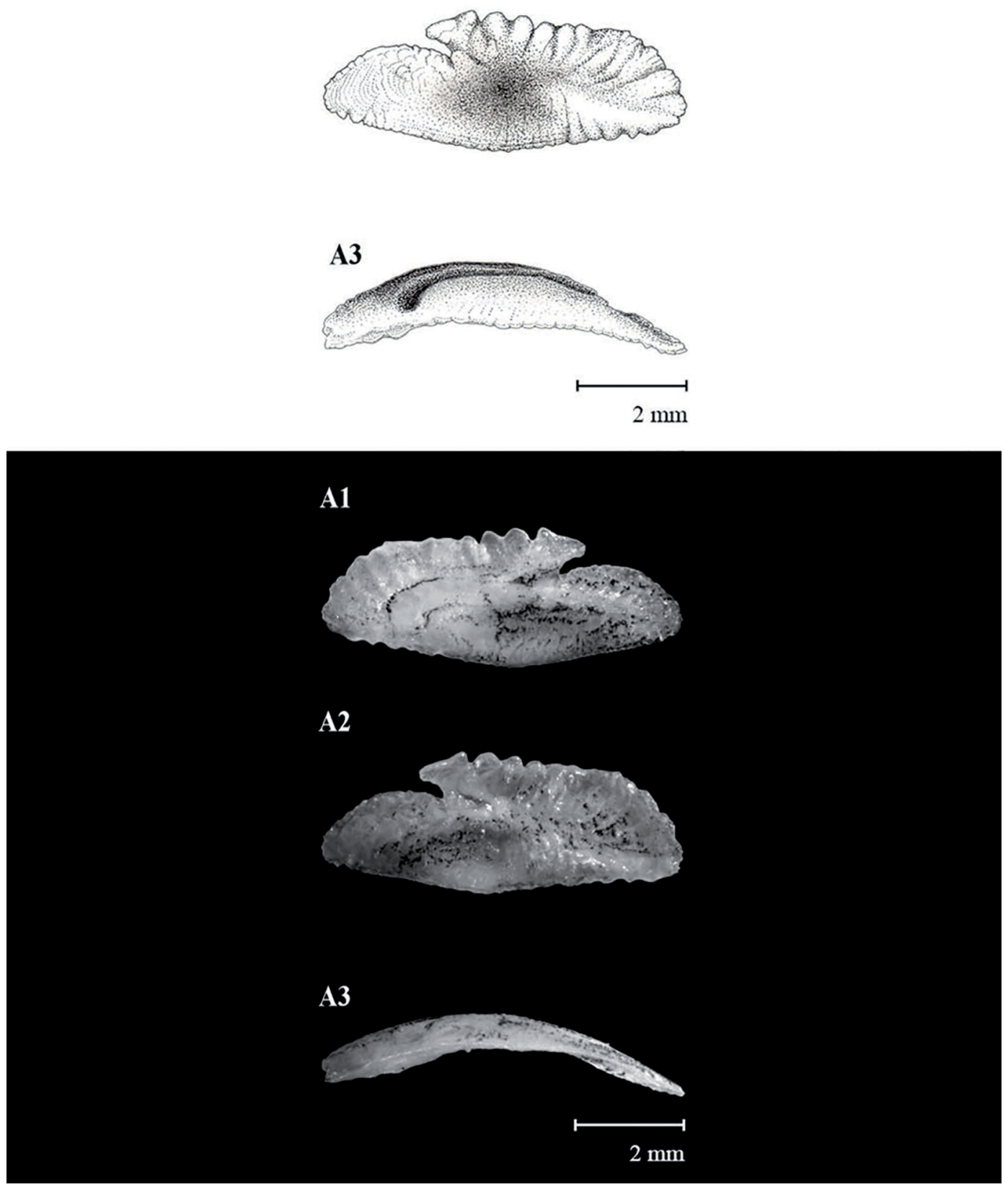

Plate 8. Illustrations (above) and photos (below) of Kyphosus incisor otolith from a fish with $254 \mathrm{~mm}$ total length. The medial face is shown in A1; the lateral face in A2; and the ventral profile in A3 (Illustrations: Laura Montserrat; Photos: Cesar Santificetur). 


\section{A1}

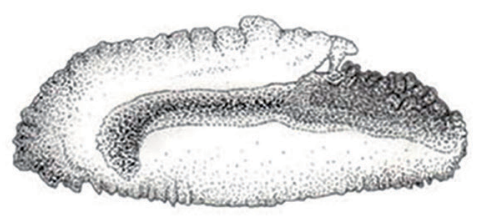

A2

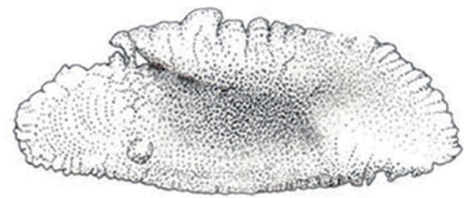

B1

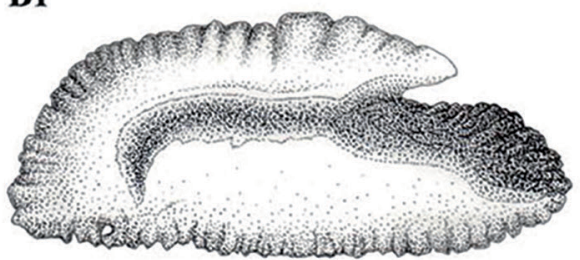

B2

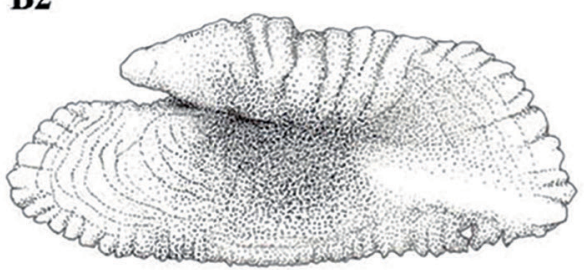

B3

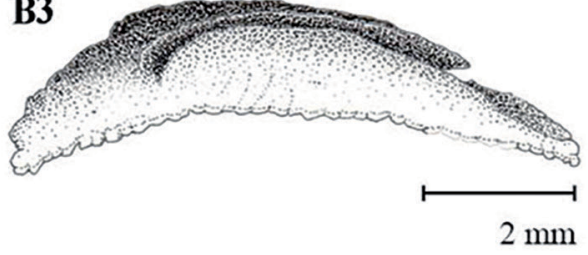

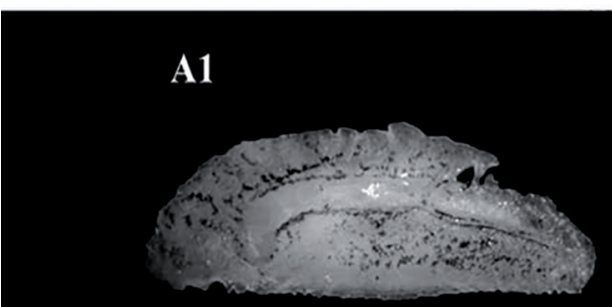

\section{B1}

A2

B2
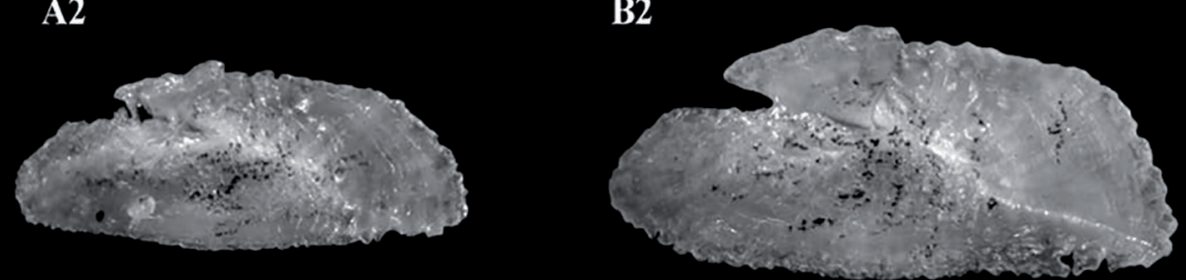

A3

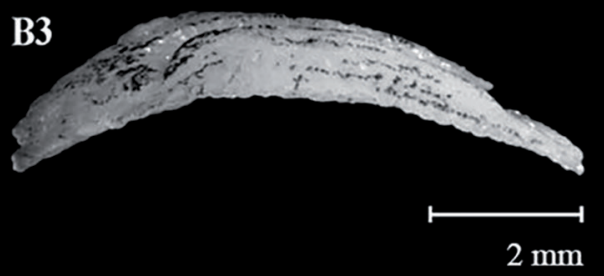

Plate 9. Illustrations (above) and photos (below) of Kyphosus sectatrix otoliths from fish with total lengths: A. $280 \mathrm{~mm}$; B. $454 \mathrm{~mm}$. The medial face is shown in A1, B1; the lateral face in A2, B2; and the ventral profile in A3, B3 (Illustrations: Laura Montserrat; Photos: Cesar Santificetur). 

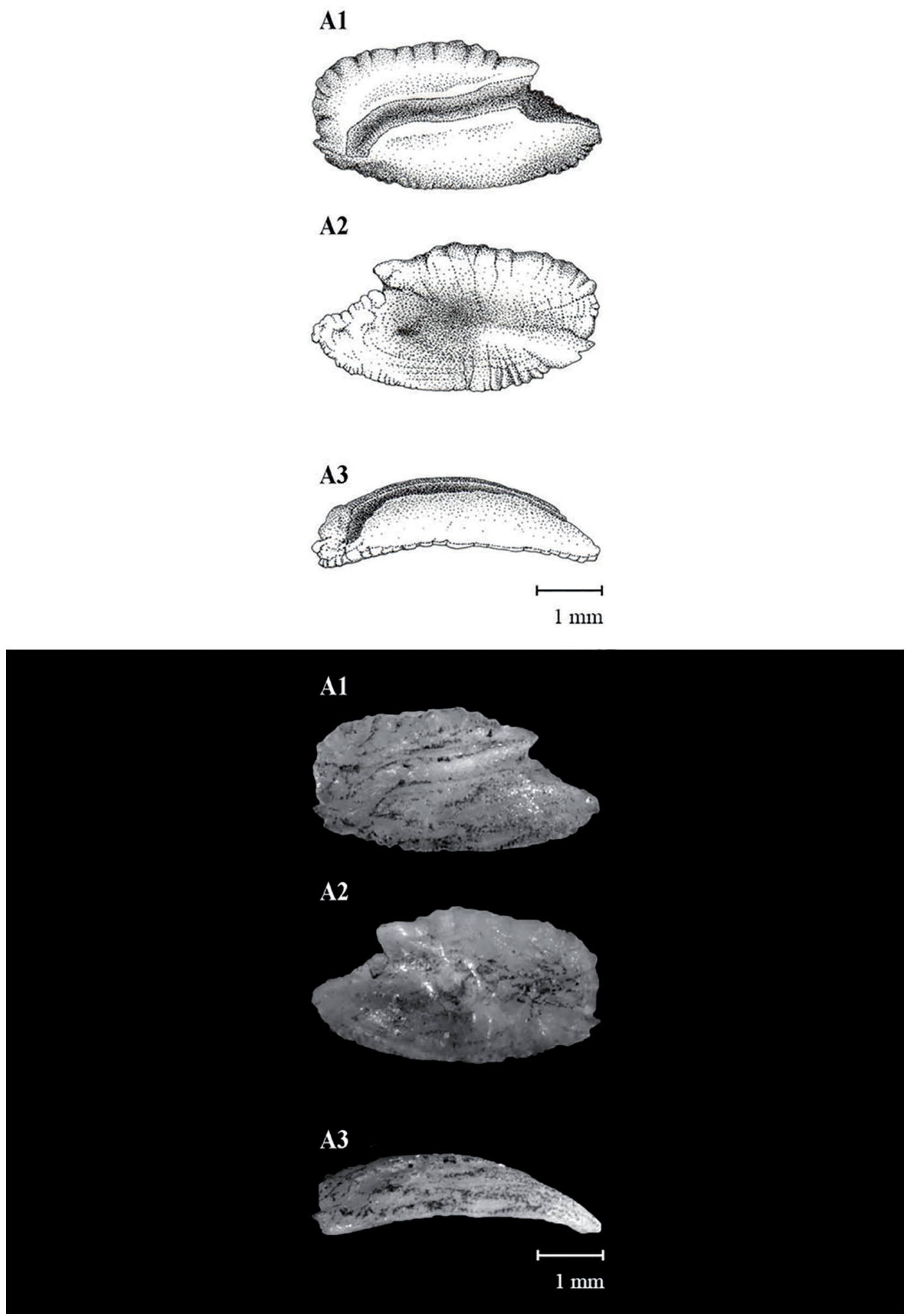

Plate 10. Illustrations (above) and photos (below) of Chaetodon striatus otolith from a fish with $149 \mathrm{~mm}$ total length. The medial face is shown in A1; the lateral face in A2; and the ventral profile in A3 (Illustrations: Laura Montserrat; Photos: Cesar Santificetur). 
A1

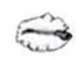

A2

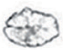

A3

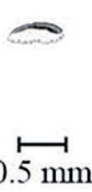

A1

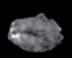

B2

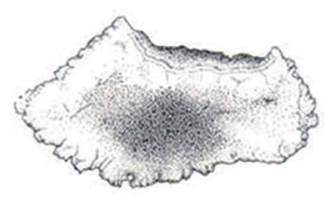

B3

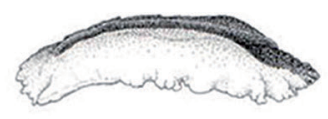

$\longmapsto 1 \mathrm{~mm}$

$0.5 \mathrm{~mm}$

B1
C1

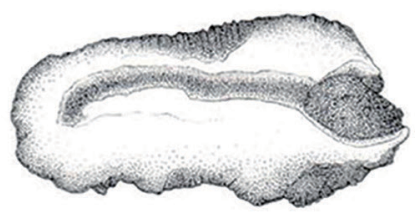

$\mathrm{C} 2$

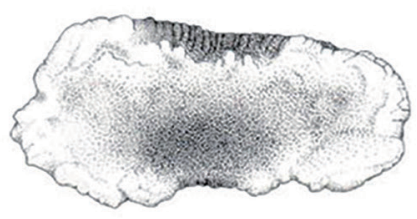

C3

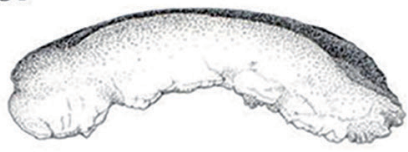

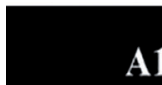

C1

$\Leftrightarrow$

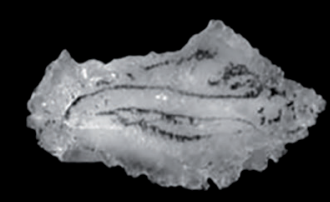

A2

B2

C2
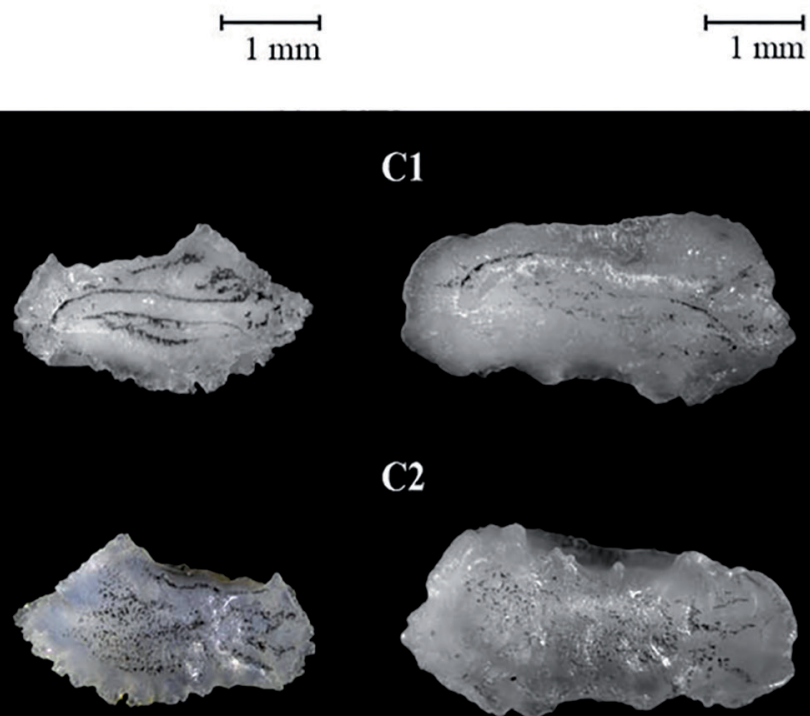

A3

B3

C3
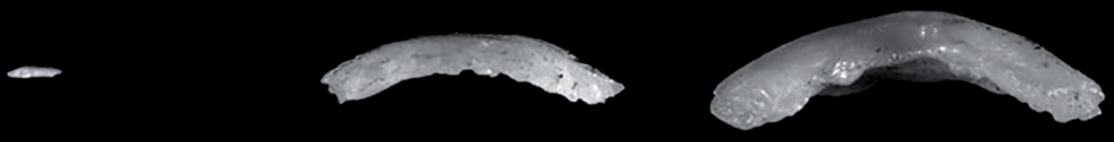

$\longmapsto 1$

$1 \mathrm{~mm}$
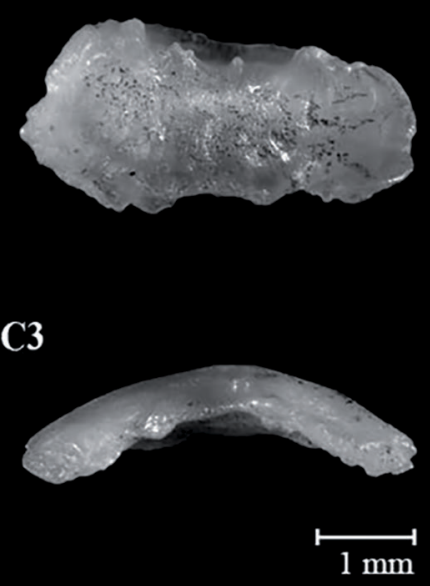

Plate 11. Illustrations (above) and photos (below) of Mugil curema otoliths from fish with total lengths: A. 29 mm; B. $259 \mathrm{~mm}$; C. $422 \mathrm{~mm}$. The medial face is shown in A1, B1, C1; the lateral face in A2, B2, C2; and the ventral profile in A3, B3, C3 (Illustrations: Laura Montserrat; Photos: Cesar Santificetur). 

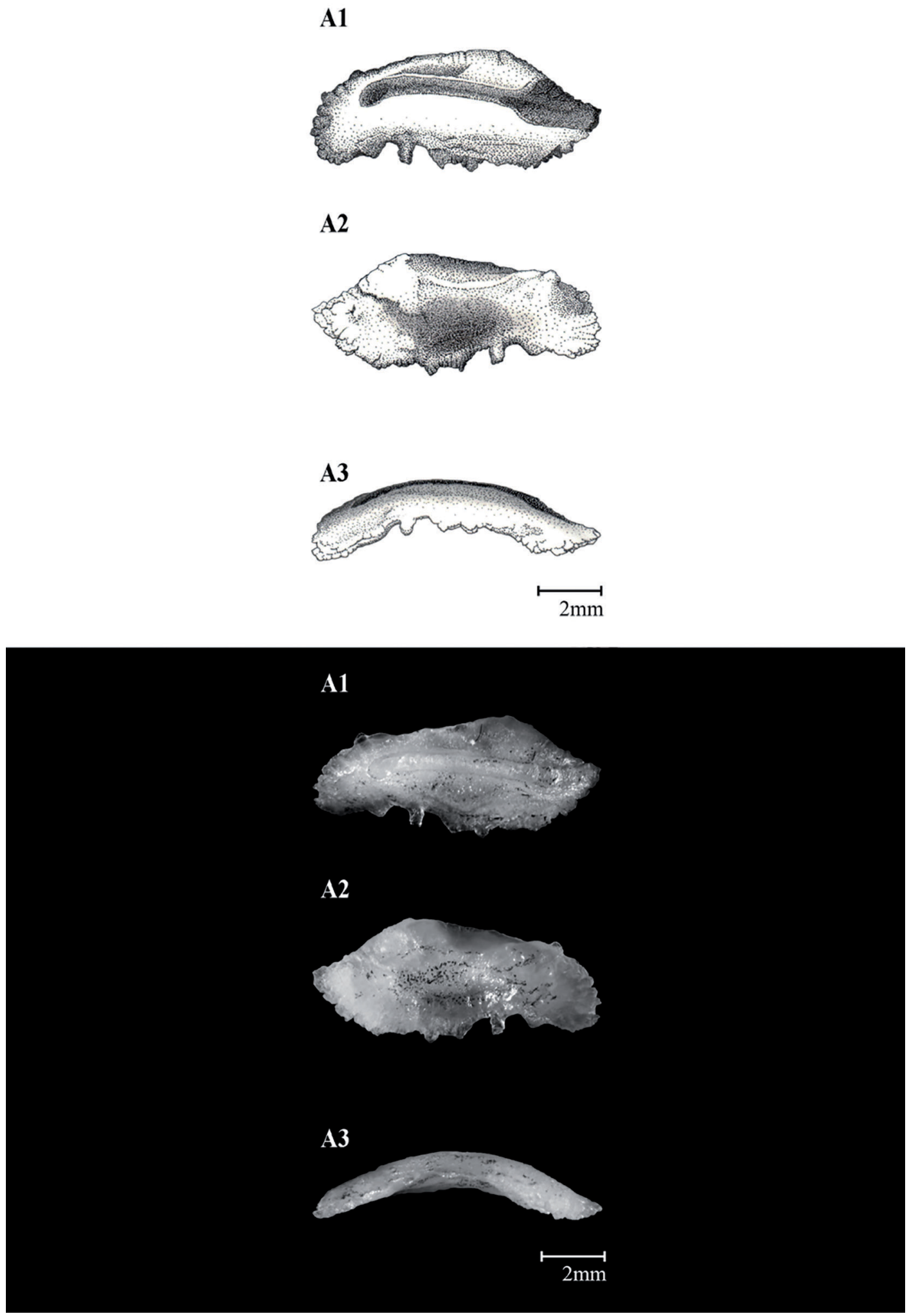

Plate 12. Illustrations (above) and photos (below) of Mugil liza otolith from a fish with $373 \mathrm{~mm}$ total length. The medial face is shown in A1; the lateral face in A2; and the ventral profile in A3 (Illustrations: Laura Montserrat; Photos: Cesar Santificetur). 
A1

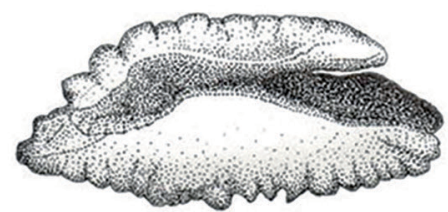

A2

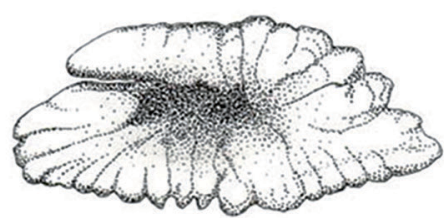

A3

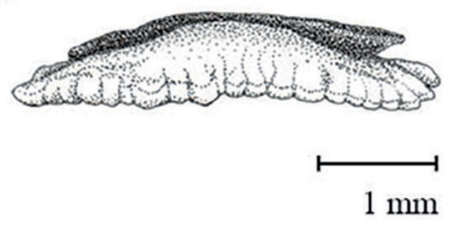

A1

A2

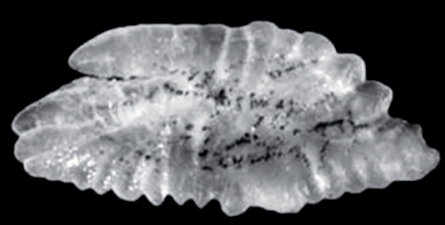

A3

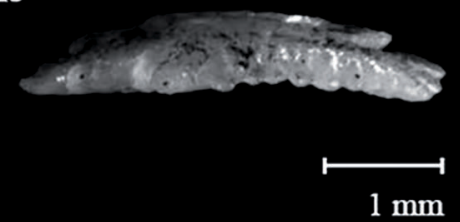

Plate 13. Illustrations (above) and photos (below) of Nicholsina usta otolith from a fish with $175 \mathrm{~mm}$ total length. The medial face is shown in A1; the lateral face in A2; and the ventral profile in A3 (Illustrations: Laura Montserrat; Photos: Cesar Santificetur). 
A1

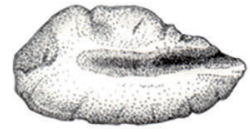

A2

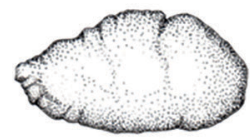

A3
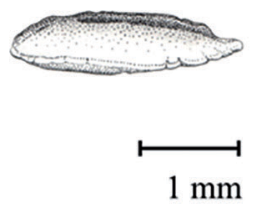

B1

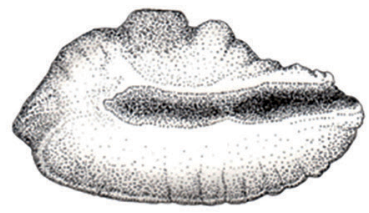

B2

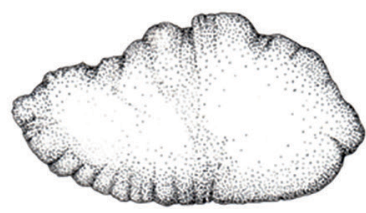

B3

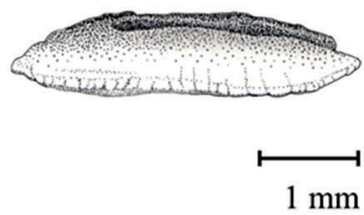

C1

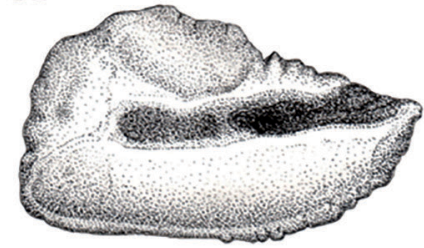

C2

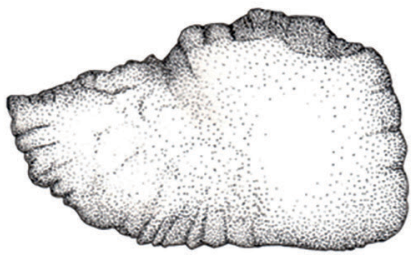

C3
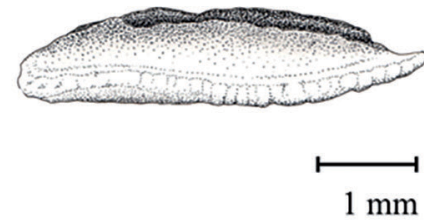

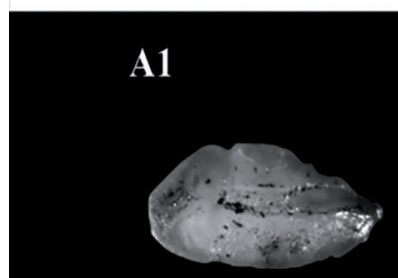

B1

C1

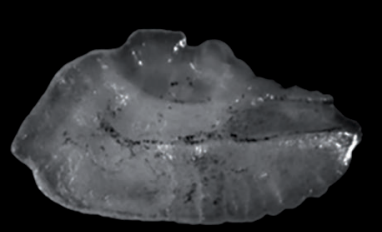

A2

B2

C2
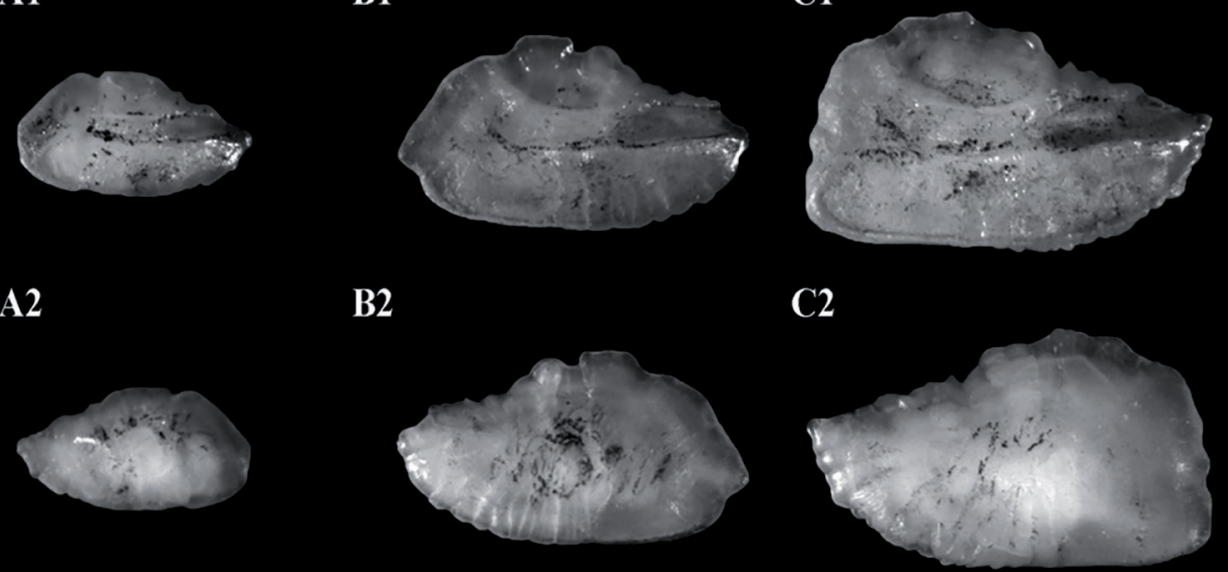

A3

B3

C3
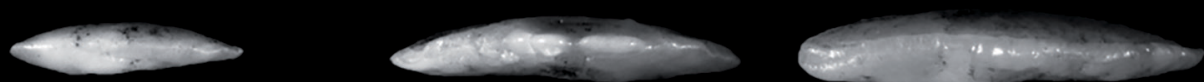

$\longmapsto$

$1 \mathrm{~mm}$
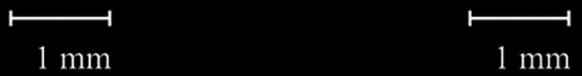

Plate 14. Illustrations (above) and photos (below) of Bembrops heterurus otoliths from fish with total lengths: A. $73 \mathrm{~mm}$; B. $134 \mathrm{~mm}$; C. $200 \mathrm{~mm}$ The medial face is shown in A1, B1, C1; the lateral face in A2, B2, C2; and the ventral profile in A3, B3, C3 (Illustrations: Laura Montserrat; Photos: Cesar Santificetur). 
A1

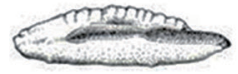

A2

A3

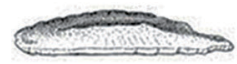

$\stackrel{\mathrm{mm}}{\rightleftarrows}$
B1

B2
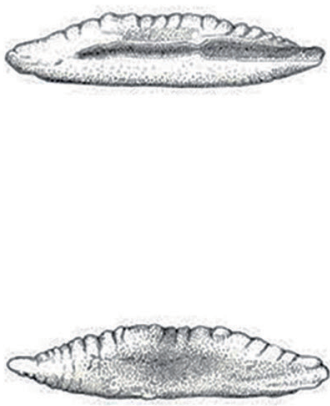

B3

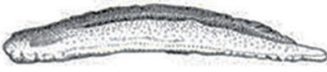

$\stackrel{\overrightarrow{m m}}{\longmapsto}$
C1

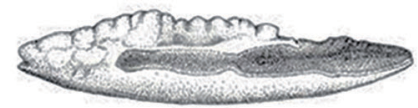

$\mathrm{C} 2$

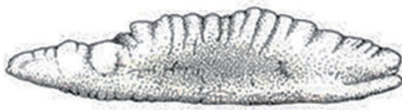

C3

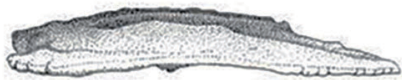

$\stackrel{\longmapsto}{2 \mathrm{~mm}}$
A1

A2

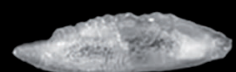

A3

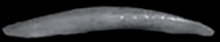

$\stackrel{\longmapsto m}{2 \mathrm{~mm}}$
B1

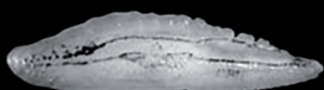

B2

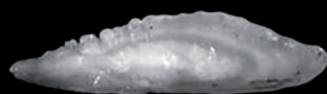

B3

C3

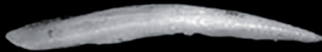

$\stackrel{1 \mathrm{~mm}}{2}$
C1

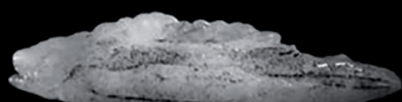

$\mathrm{C} 2$

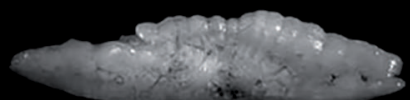

Plate 15. Illustrations (above) and photos (below) of Percophis brasiliensis otoliths from fish with total lengths: A. $284 \mathrm{~mm}$; B. $430 \mathrm{~mm}$; C. 590 $\mathrm{mm}$. The medial face is shown in A1, B1, C1; the lateral face in A2, B2, C2; and the ventral profile in A3, B3, C3 (Illustrations: Laura Montserrat; Photos: Cesar Santificetur). 
A1

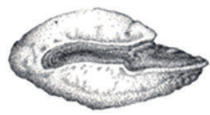

A2

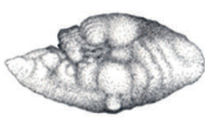

A3

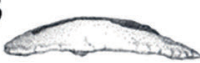

B1

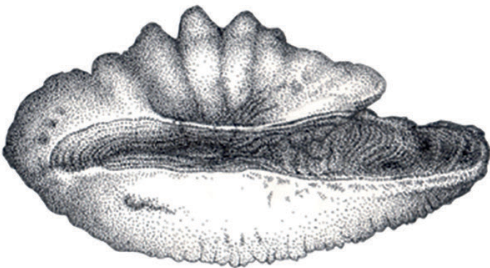

B2

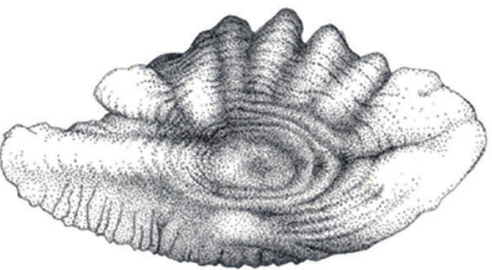

B3

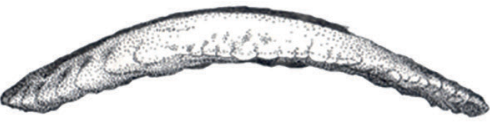

$2 \mathrm{~mm}$

$2 \mathrm{~mm}$

A1

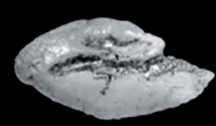

A2

A3

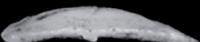

B1

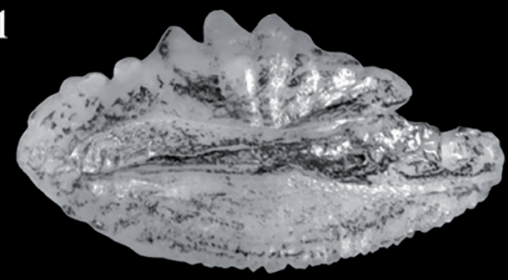

B2

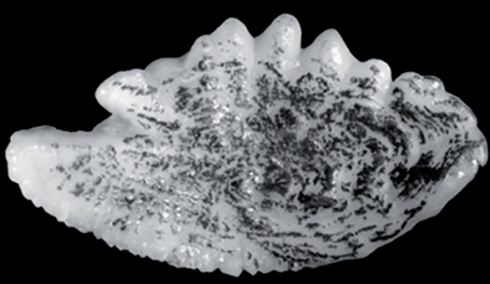

Plate 16. Illustrations (above) and photos (below) of Pseudopercis numida otoliths from fish with total lengths: A. 128 mm; B. 467 mm. The medial face is shown in A1, B1; the lateral face in A2, B2; and the ventral profile in A3, B3 (Illustrations and Photos: Alexandre Arackawa). 

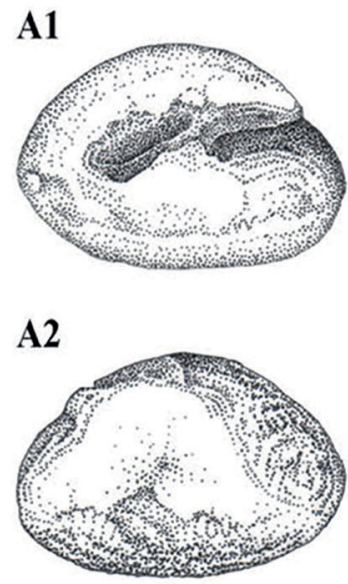

\section{A3}

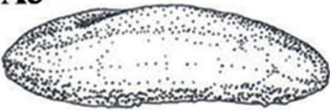

\section{$0,5 \mathrm{~mm}$}

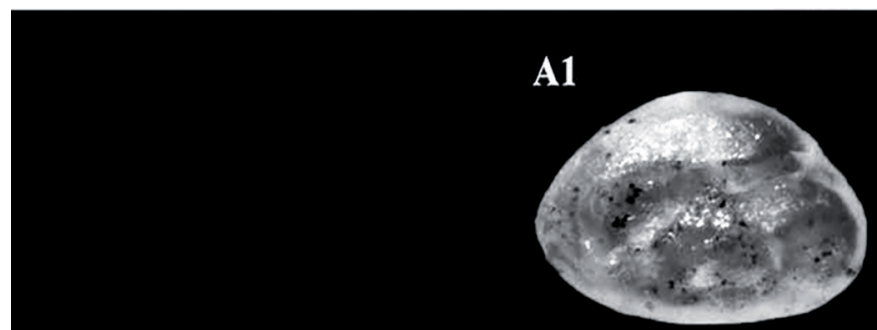

\section{A2}
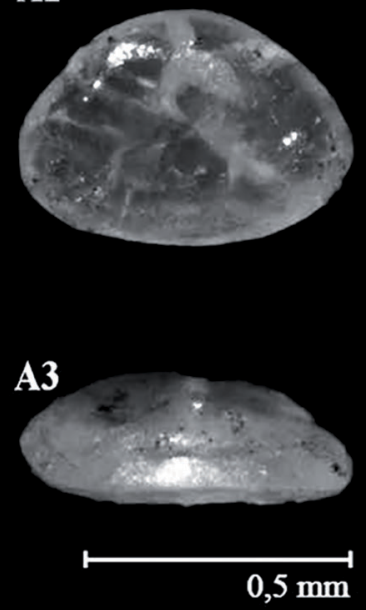

Plate 17. Illustrations (above) and photos (below) of Scartella cristata otolith from a fish with $22 \mathrm{~mm}$ total length. The medial face is shown in A1; the lateral face in A2; and the ventral profile in A3 (Illustrations: Silvia de Almeida Gonsales; Photos: Cesar Santificetur). 
A1

(3)

A2

A3

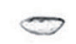

B1

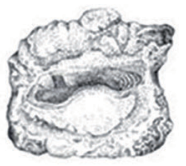

B2

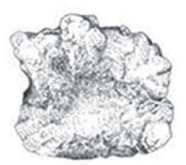

B3

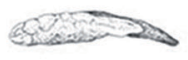

C1

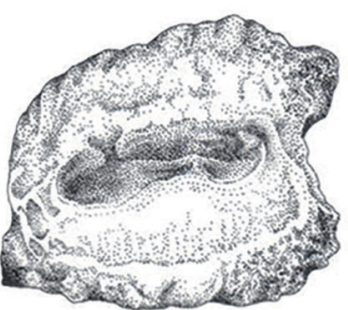

C2

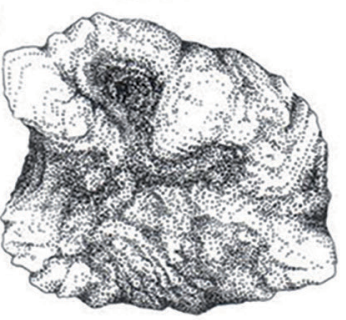

C3

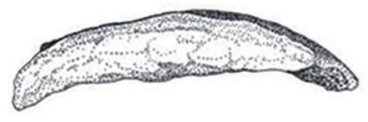

$\stackrel{\longmapsto}{0,5 \mathrm{~mm}}$

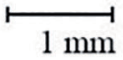

$\longmapsto 1 \mathrm{~mm}$

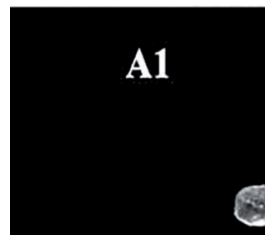

B1

8

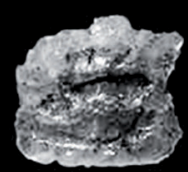

C1

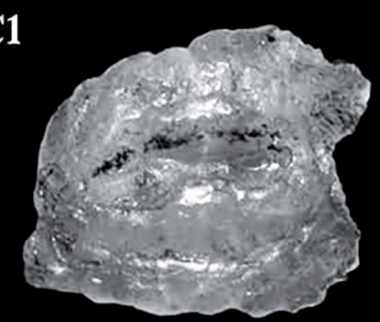

A2

B2

C2
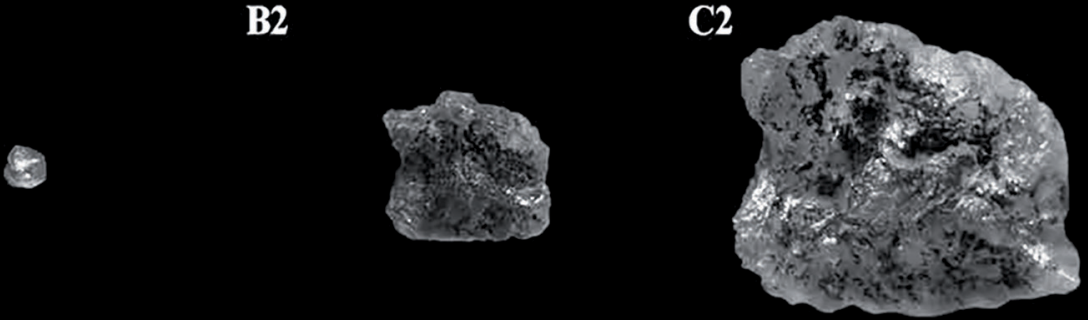

A3

B3

C3
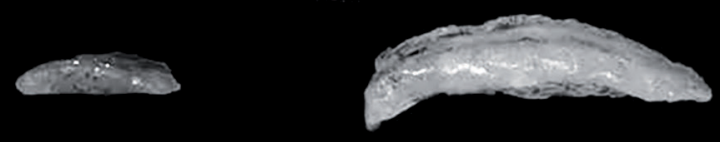

$0, \stackrel{\longmapsto}{\mathrm{mm}}$

$\stackrel{1 \mathrm{~mm}}{\rightleftharpoons}$

$1 \mathrm{~mm}$

Plate 18. Illustrations (above) and photos (below) of Bathygobius soporator otoliths from fish with total lengths: A. $13 \mathrm{~mm}$; B. $75 \mathrm{~mm}$; C. 131 $\mathrm{mm}$. The medial face is shown in A1, B1, C1; the lateral face in A2, B2, C2; and the ventral profile in A3, B3, C3 (Illustrations: Silvia de Almeida Gonsales; Photos: Cesar Santificetur). 
A1

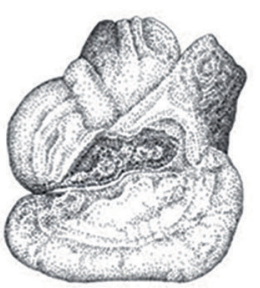

A2

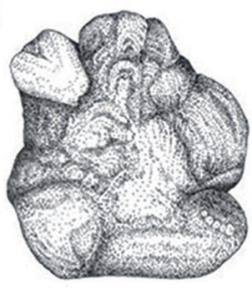

A3

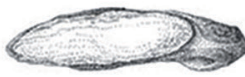

$1 \mathrm{~mm}$

\1

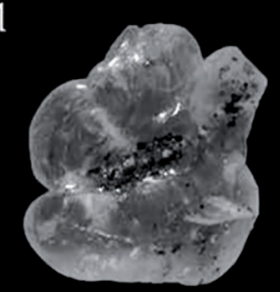

$\Delta 2$

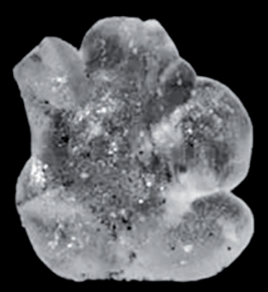

13

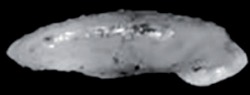

$1 \mathrm{~mm}$

Plate 19. Illustrations (above) and photos (below) of Ctenogobius smaragdus otolith from a fish with $45 \mathrm{~mm}$ total length. The medial face is shown in A1; the lateral face in A2; and the ventral profile in A3 (Illustrations: Silvia de Almeida Gonsales; Photos: Cesar Santificetur). 
A1

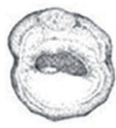

A2

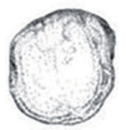

A3
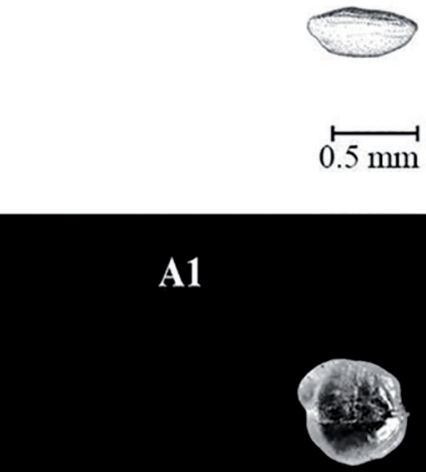

A2

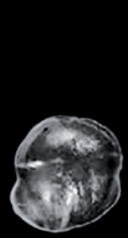

A3

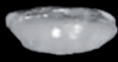

B3

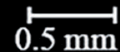

B1

B2
B1

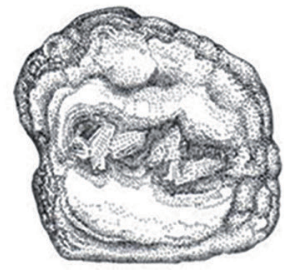

B2

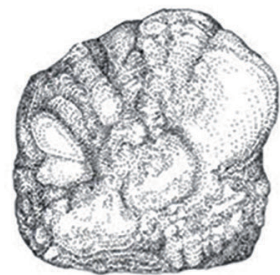

B3

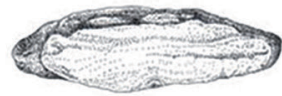

$\stackrel{\longmapsto}{0.5 \mathrm{~mm}}$
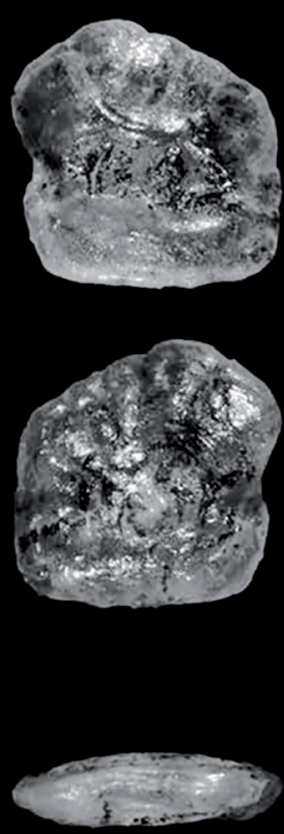

$\stackrel{\longmapsto \mathrm{mm}}{0.5 \mathrm{~m}}$

Plate 20. Illustrations (above) and photos (below) of Ctenogobius boleosoma otoliths from fish with total lengths: A. $17 \mathrm{~mm}$; B. $55 \mathrm{~mm}$. The medial face is shown in A1, B1; the lateral face in A2, B2; and the ventral profile in A3, B3 (Illustrations: Silvia de Almeida Gonsales; Photos: Cesar Santificetur). 
A1

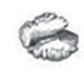

A2

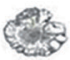

A3

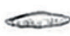

$1 \mathrm{~mm}$
B1

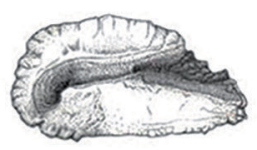

B2

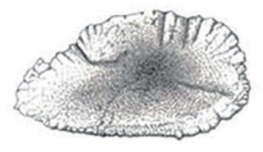

B3
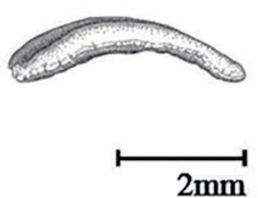

C1

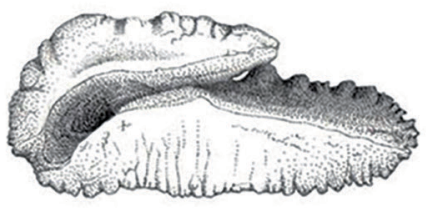

C2

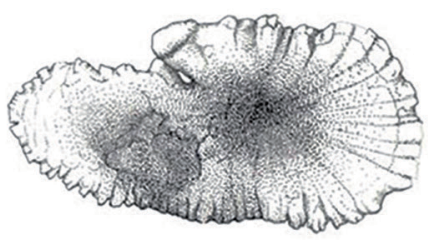

C3

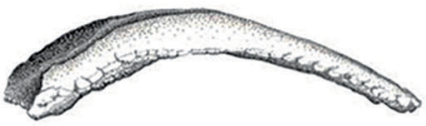

A1

B1

C1
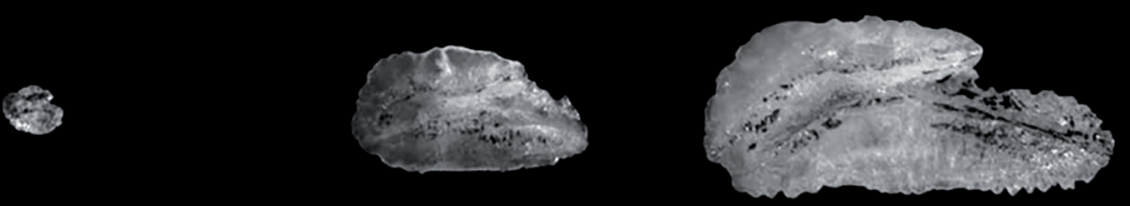

A2

B2

C2
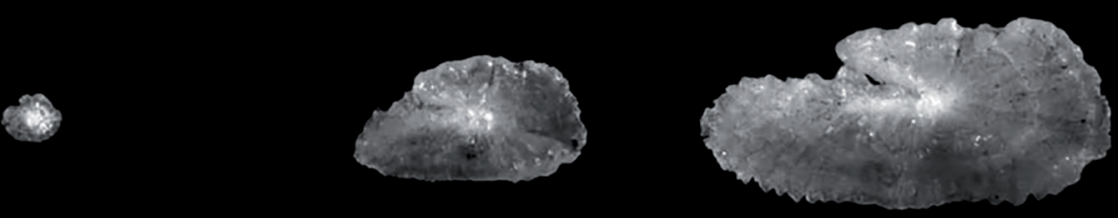

A3

B3

C3

20

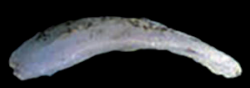

$1 \mathrm{~mm}$
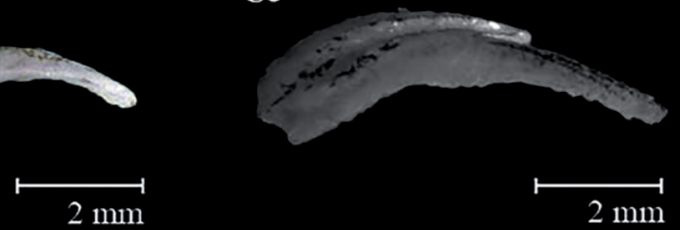

Plate 21. Illustrations (above) and photos (below) of Chaetodipterus faber otoliths from fish with total lengths: A. 20 mm; B. 114 mm; C. 283 $\mathrm{mm}$. The medial face is shown in A1, B1, C1; the lateral face in A2, B2, C2; and the ventral profile in A3, B3, C3 (Illustrations: Laura Montserrat; Photos: Cesar Santificetur). 
A1

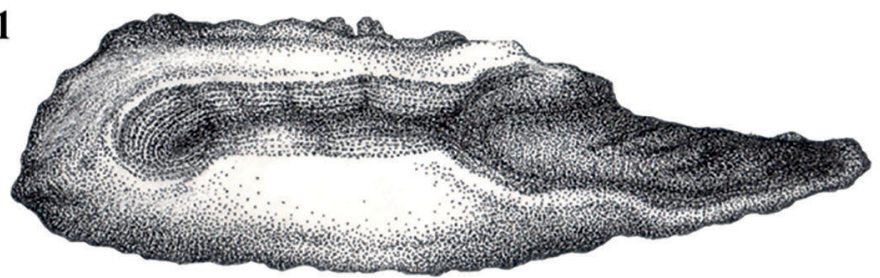

A2

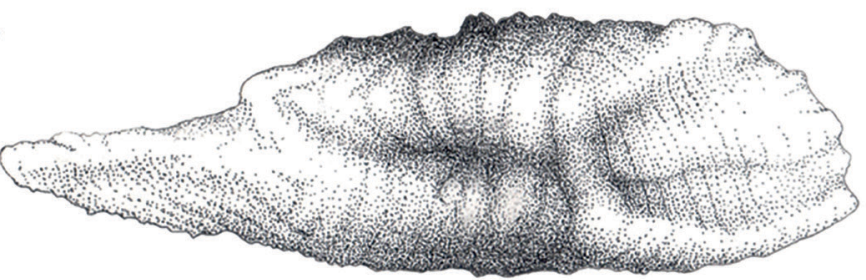

A3
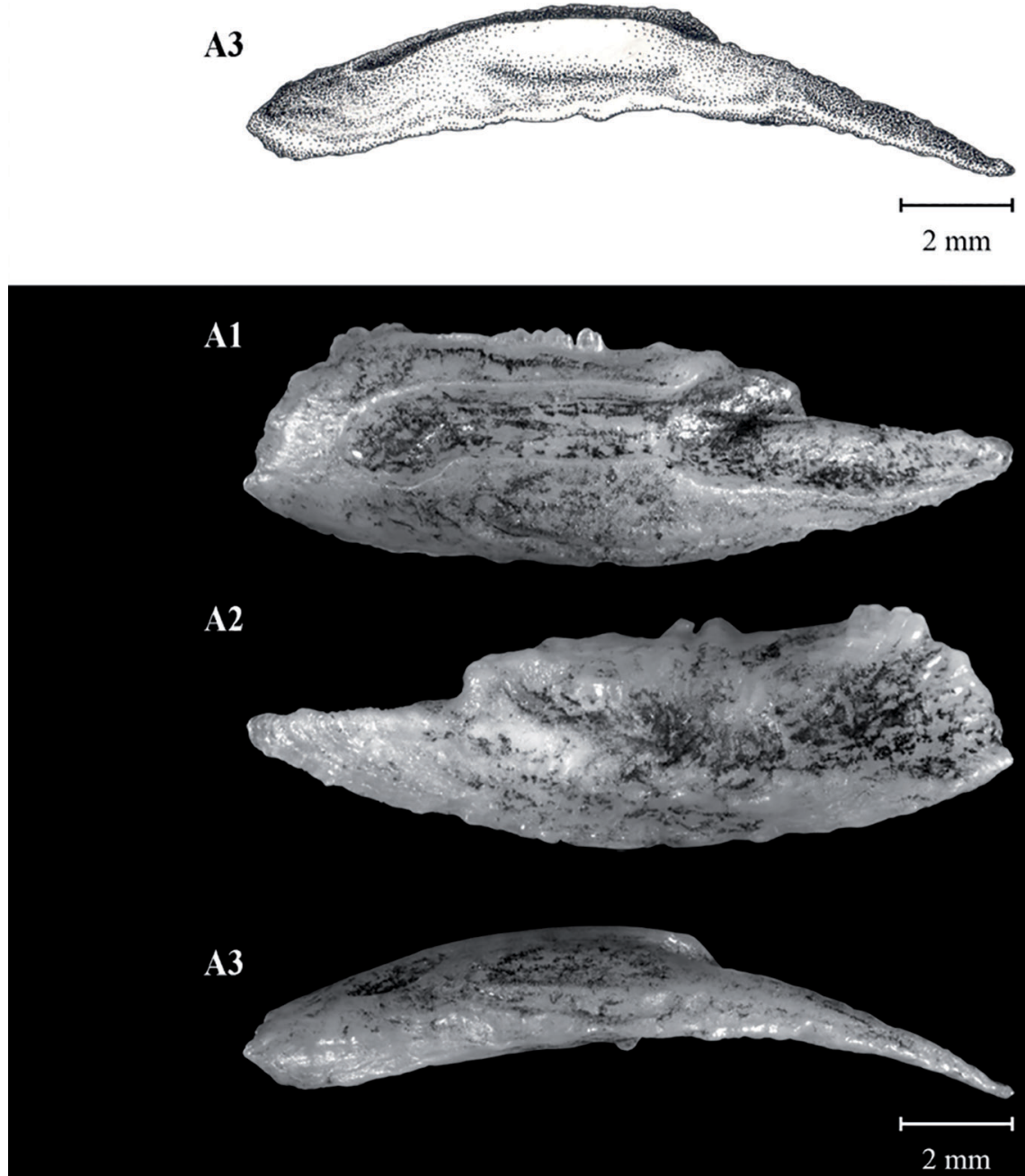

Plate 22. Illustrations (above) and photos (below) of Sphyraena barracuda otoliths from a fish with $563 \mathrm{~mm}$ total lengths. The medial face is shown in A1; the lateral face in A2; and the ventral profile in A3 (Illustrations and Photos: Alexandre Arackawa). 
A1

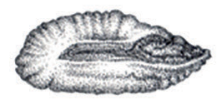

A2

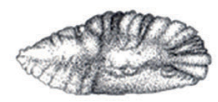

A3

erivindives
B1

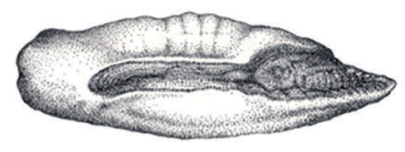

B2

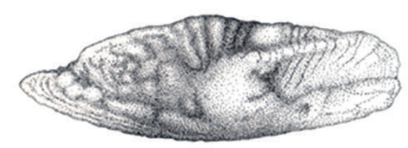

C1

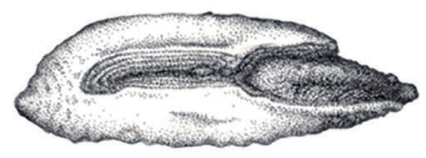

C2

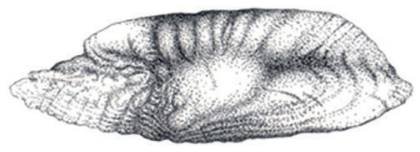

C3
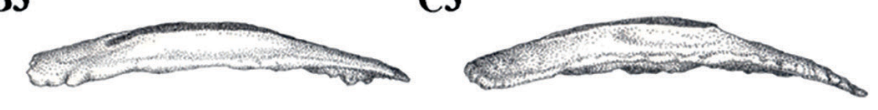
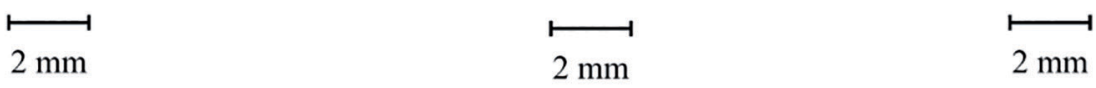

$2 \mathrm{~mm}$

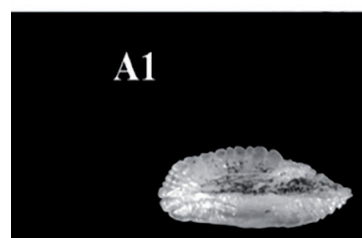

B1

C1

A2

B2

C2
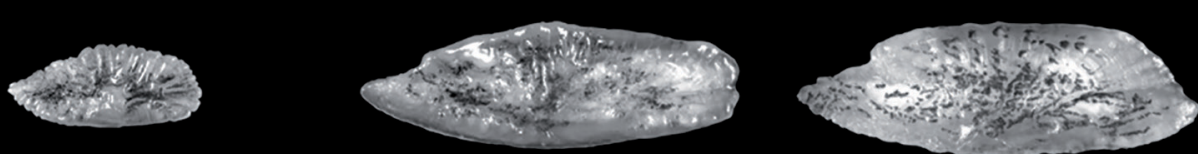

A3

B3

C3

$\longrightarrow$
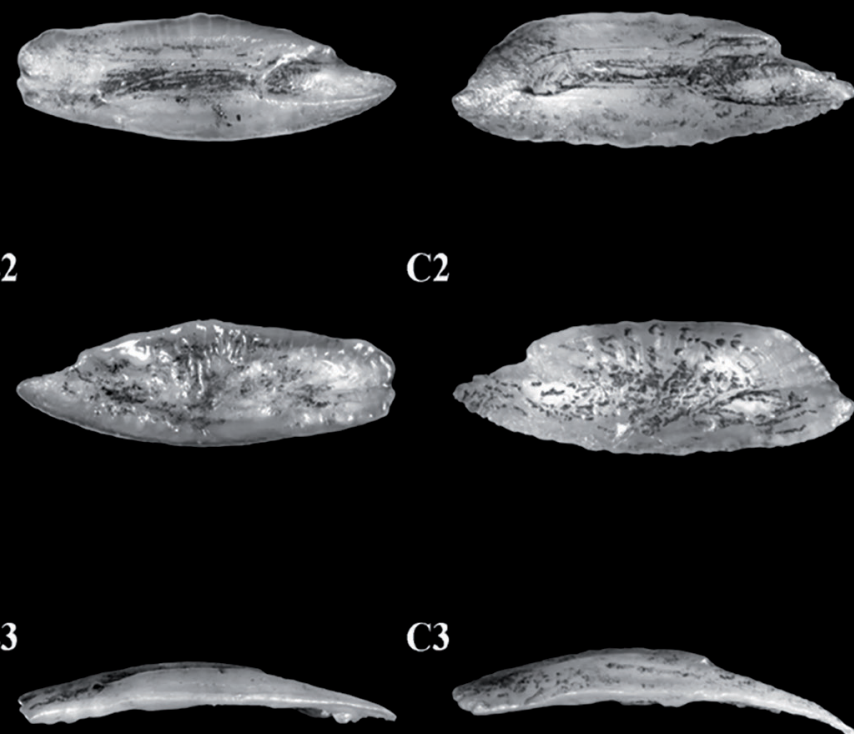

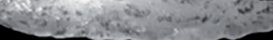

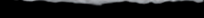

Plate 23. Illustrations (above) and photos (below) of Sphyraena guachancho otoliths from fish with total lengths: A. $140 \mathrm{~mm}$; B. 309 mm; C. $455 \mathrm{~mm}$. The medial face is shown in A1, B1, C1; the lateral face in A2, B2, C2; and the ventral profile in A3, B3, C3 (Illustrations and Photos: Alexandre Arackawa). 

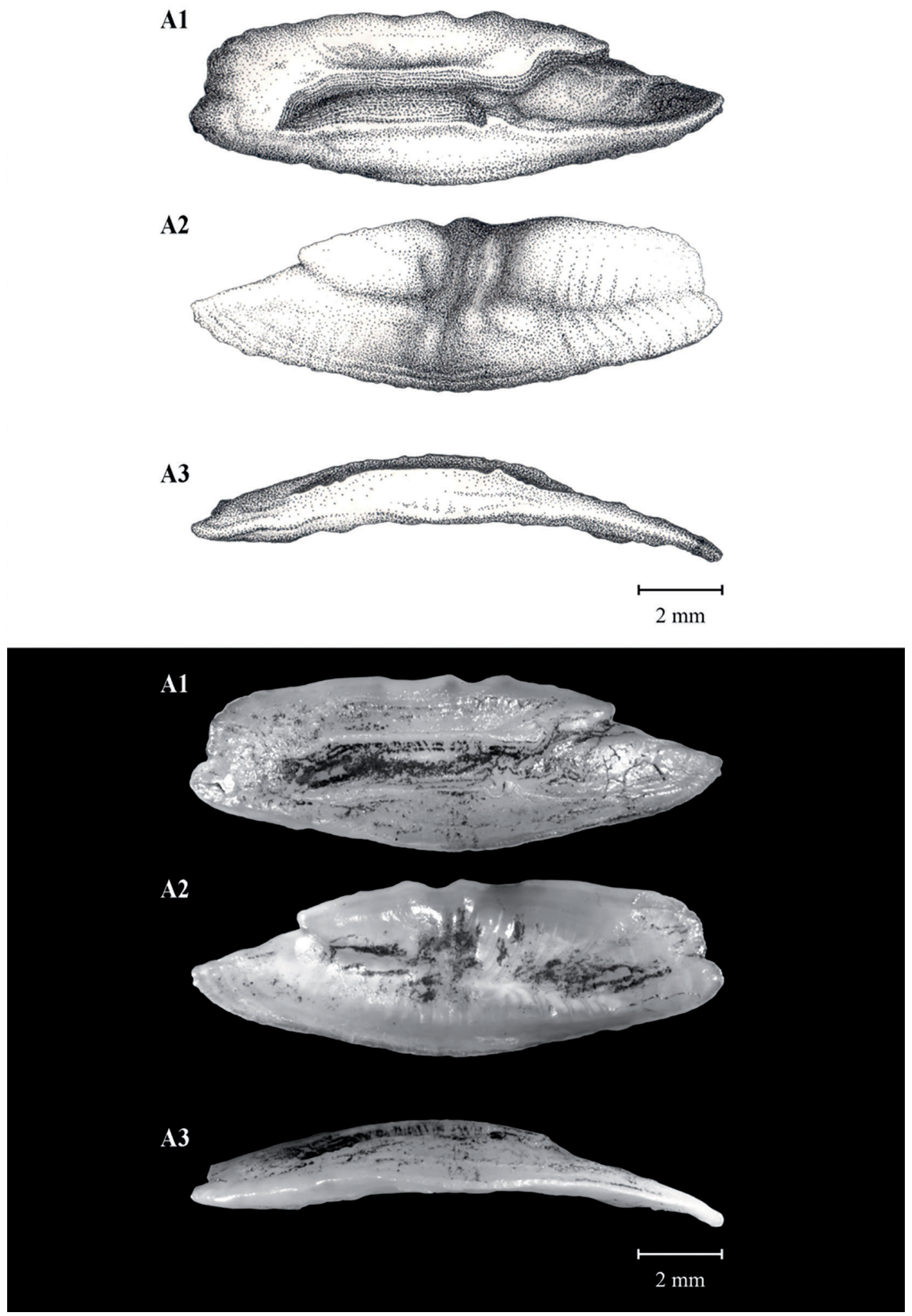

Plate 24. Illustrations (above) and photos (below) of Sphyraena tome otolith from a fish with $412 \mathrm{~mm}$ total length. The medial face is shown in A1; the lateral face in A2; and the ventral profile in A3 (Illustrations and Photos: Alexandre Arackawa). 
A1

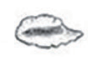

A2

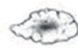

A3

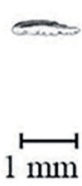

B1

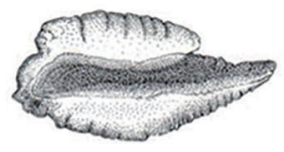

B2

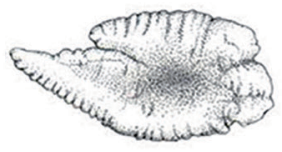

B3

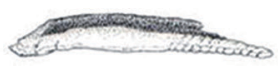

$1 \mathrm{~mm}$
C1

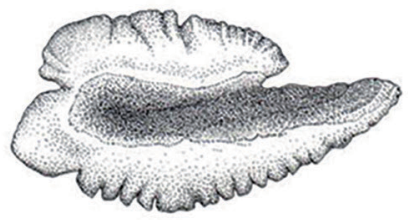

C2

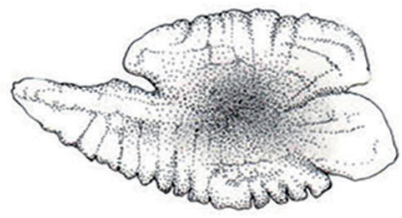

C3

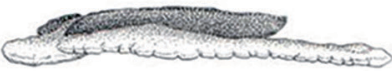

A1

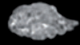

A2

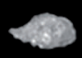

A3
B1

B2

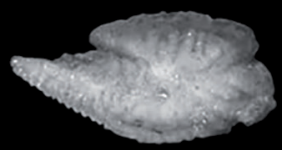

B3
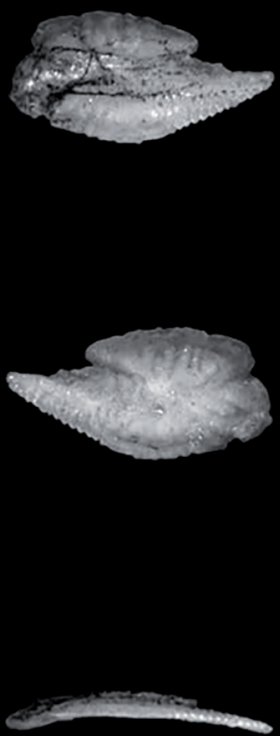

C1

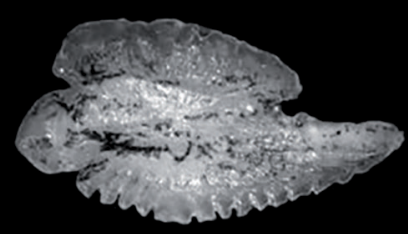

C2

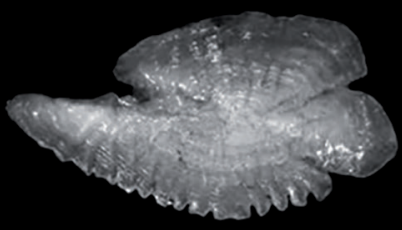

C3

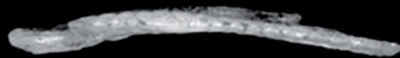

$1 \mathrm{~mm}$

Plate 25. Illustrations (above) and photos (below) of Thyrsitops lepidopoides otoliths from fish with total length: A. 29 mm; B. $197 \mathrm{~mm}$; C. 308 $\mathrm{mm}$. The medial face is shown in A1, B1, C1; the lateral face in A2, B2, C2; and the ventral profile in A3, B3, C3 (Illustrations: Laura Montserrat; Photos: Cesar Santificetur). 
A1

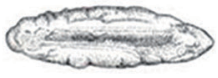

A2

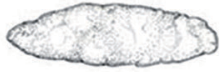

A3

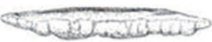

B1

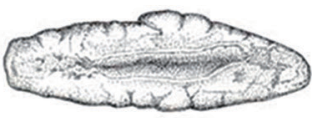

B2

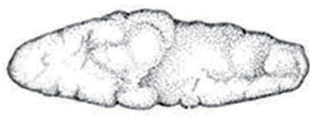

B3

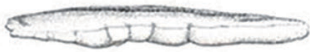

C1

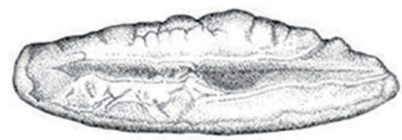

$\mathrm{C} 2$

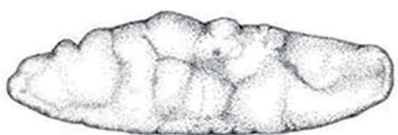

C3

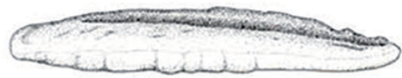

A1

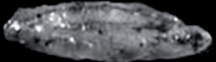

$1 \mathrm{~mm}$

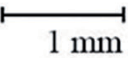

B1

B2

B3

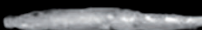

$1 \mathrm{~mm}$

A3

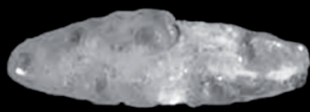

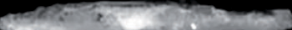

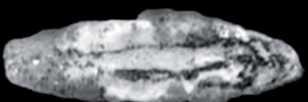

C1

C2

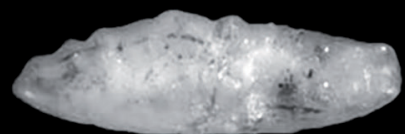

C3

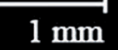

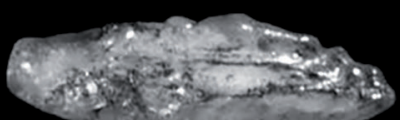

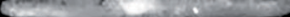

$1 \mathrm{~mm}$

\section{$1 \mathrm{~mm}$}

Plate 26. Illustrations (above) and photos (below) of Benthodesmus elongates otoliths from fish with total lengths: A. 259 mm; B. 370 mm; C. 494 $\mathrm{mm}$. The medial face is shown in A1, B1, C1; the lateral face in A2, B2, C2; and the ventral profile in A3, B3, C3 (Illustrations: Laura Montserrat; Photos: Cesar Santificetur). 
A1

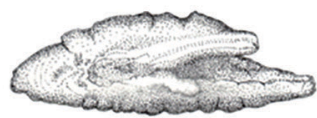

A2

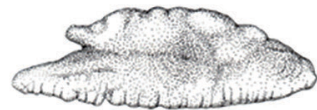

A3
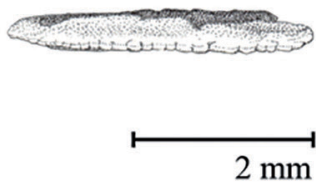

A1

A2

A3

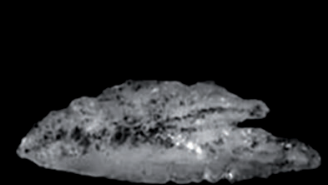

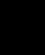

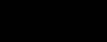

2

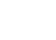

$2 \mathrm{~mm}$

\section{B1}

B2

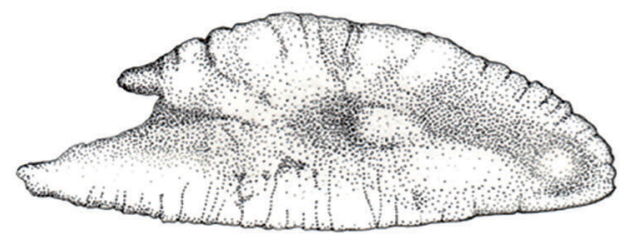

B3

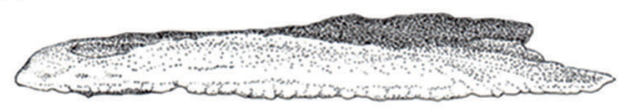

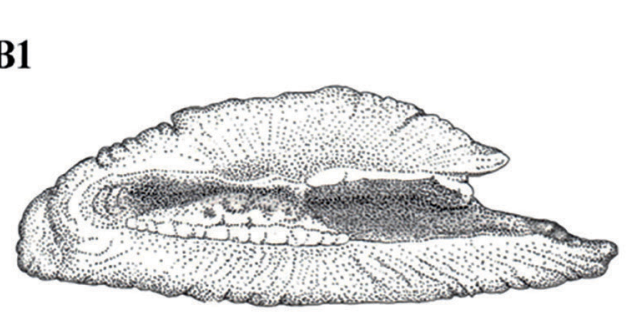

$2 \mathrm{~mm}$
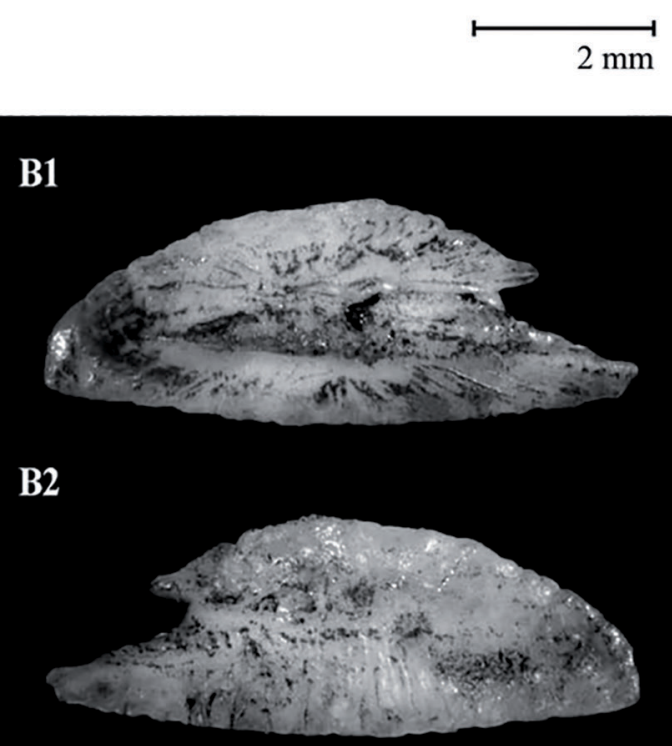

B3

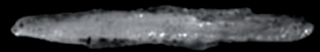

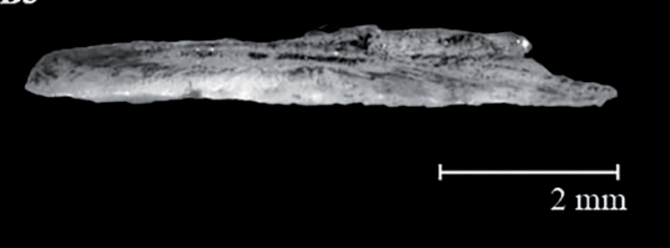

Plate 27. Illustrations (above) and photos (below) of Evoxymetopon taeniatus otoliths from fish with total lengths: A. $512 \mathrm{~mm}$; B. $1640 \mathrm{~mm}$. The medial face is shown in A1, B1; the lateral face in A2, B2; and the ventral profile in A3, B3 (Illustrations: Laura Montserrat; Photos: Cesar Santificetur). 
A1

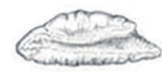

A2

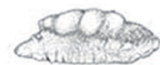

A3
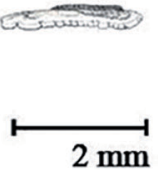

A1

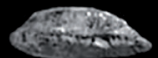

A2

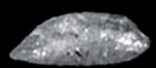

A3
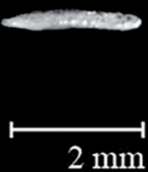

B1

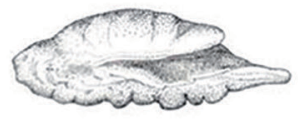

B2

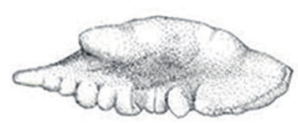

B3
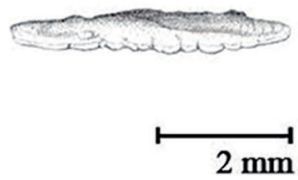

B1

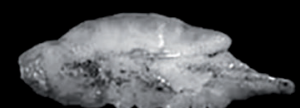

C3
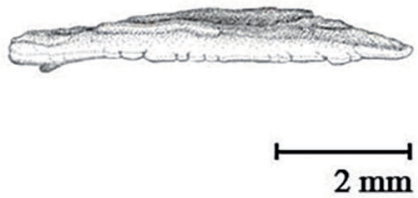

C1

C2

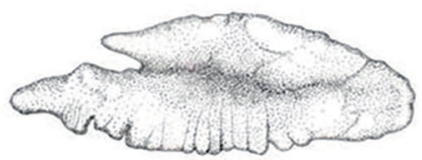

C1

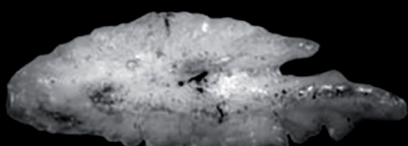

C2
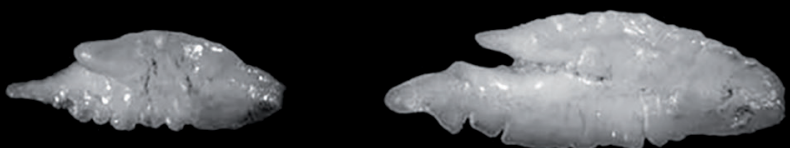

Plate 28. Illustrations (above) and photos (below) of Lepidopus altifrons otoliths from fish with total length: A. $256 \mathrm{~mm}$; B. $530 \mathrm{~mm}$; C. $795 \mathrm{~mm}$. The medial face is shown in A1, B1, C1; the lateral face in A2, B2, C2; and the ventral profile in A3, B3, C3 (Illustrations: Laura Montserrat; Photos: Cesar Santificetur). 
A1

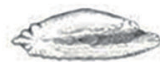

A2

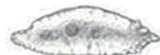

A3

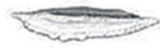

$\stackrel{\longmapsto}{2 \mathrm{~mm}}$
B1

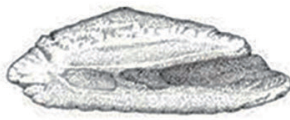

B2

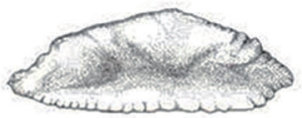

B3

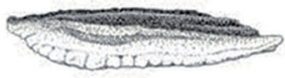

$\stackrel{\longmapsto}{2 \mathrm{~mm}}$
C1

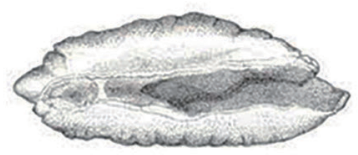

C2

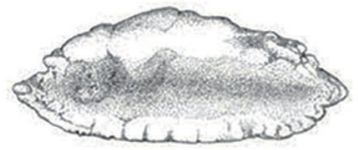

C3

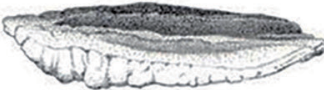

$\stackrel{\longmapsto}{2 \mathrm{~mm}}$
A1

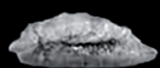

A2

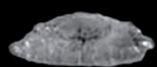

A3

penersy $2 \mathrm{~mm}$
B1

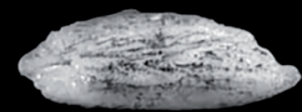

B2

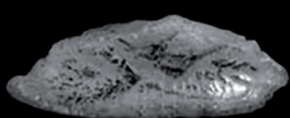

B3

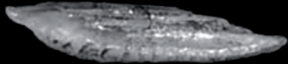


C1

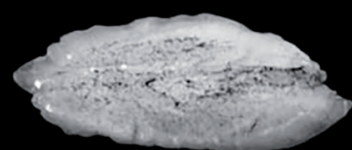

$\mathrm{C} 2$

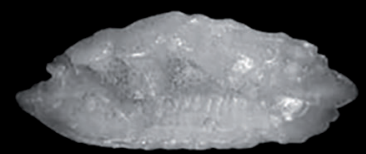

C3

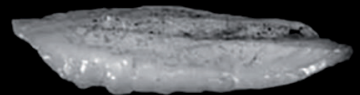

$2 \mathrm{~mm}$

Plate 29. Illustrations (above) and photos (below) of Trichiurus lepturus otoliths from fish with total lengths: A. 745 mm; B. 903 mm; C. 1825 $\mathrm{mm}$. The medial face is shown in A1, B1, C1; the lateral face in A2, B2, C2; and the ventral profile in A3, B3, C3 (Illustrations: Laura Montserrat; Photos: Cesar Santificetur). 
A1

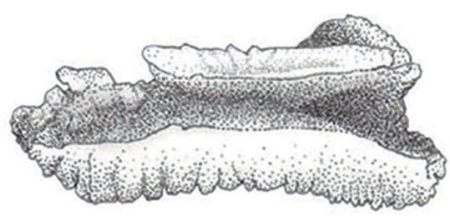

A2

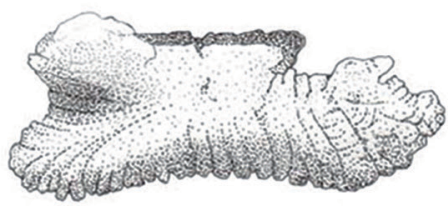

A3

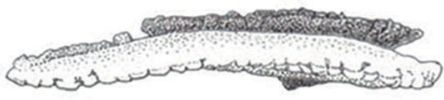

$1 \mathrm{~mm}$

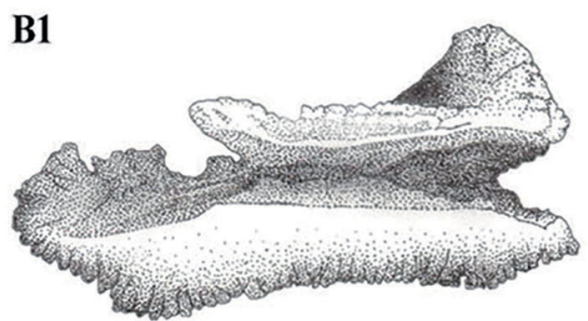

B2

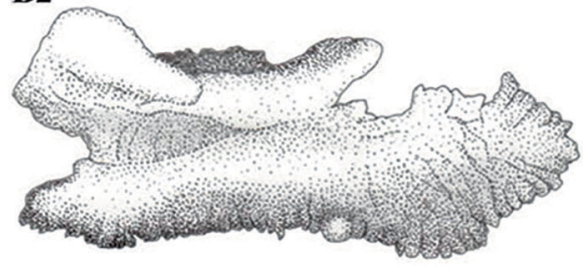

B3

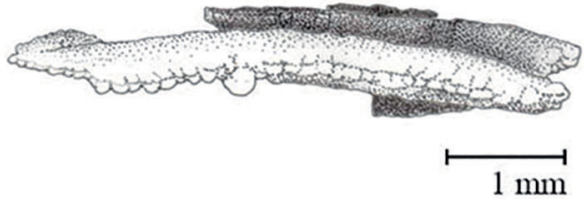

\section{A1}

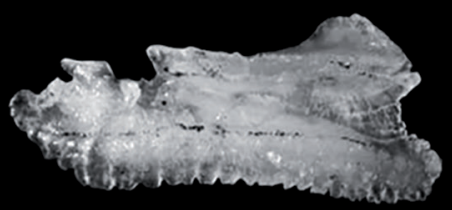

A2

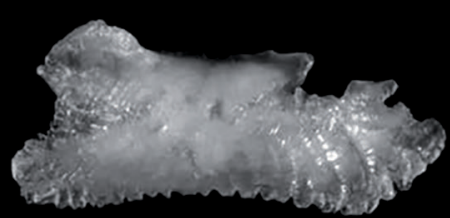

A3

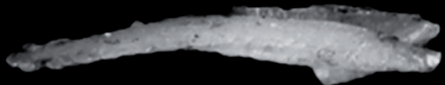

B1

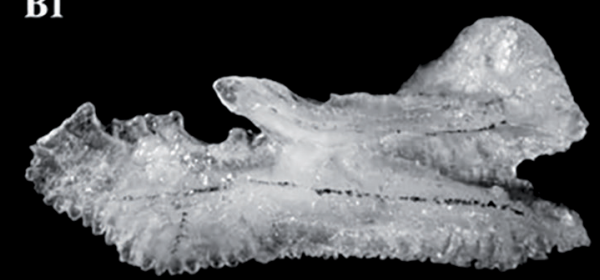

B2

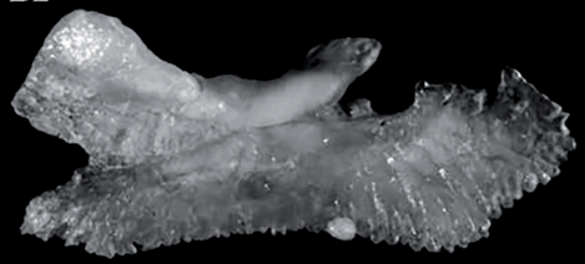

B3

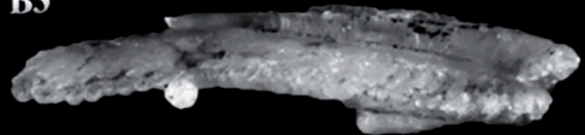

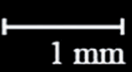

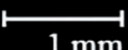

Plate 30. Illustrations (above) and photos (below) of Katsuwonus pelamis otoliths from fish with total lengths: A. $375 \mathrm{~mm}$; B. $473 \mathrm{~mm}$. The medial face is shown in A1, B1; the lateral face in A2, B2; and the ventral profile in A3, B3 (Illustrations: Laura Montserrat; Photos: Cesar Santificetur). 
A1

(s)

A2

(1)

A3

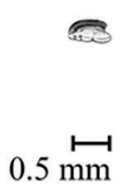

A1

\section{2}

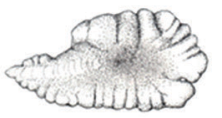

B3

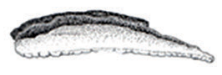

$\longmapsto$
C1

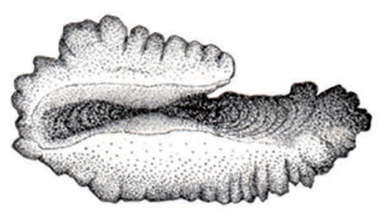

C2

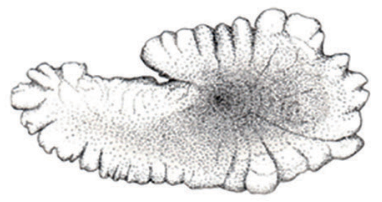

C3

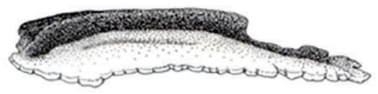

$\stackrel{\longmapsto}{1 \mathrm{~mm}}$

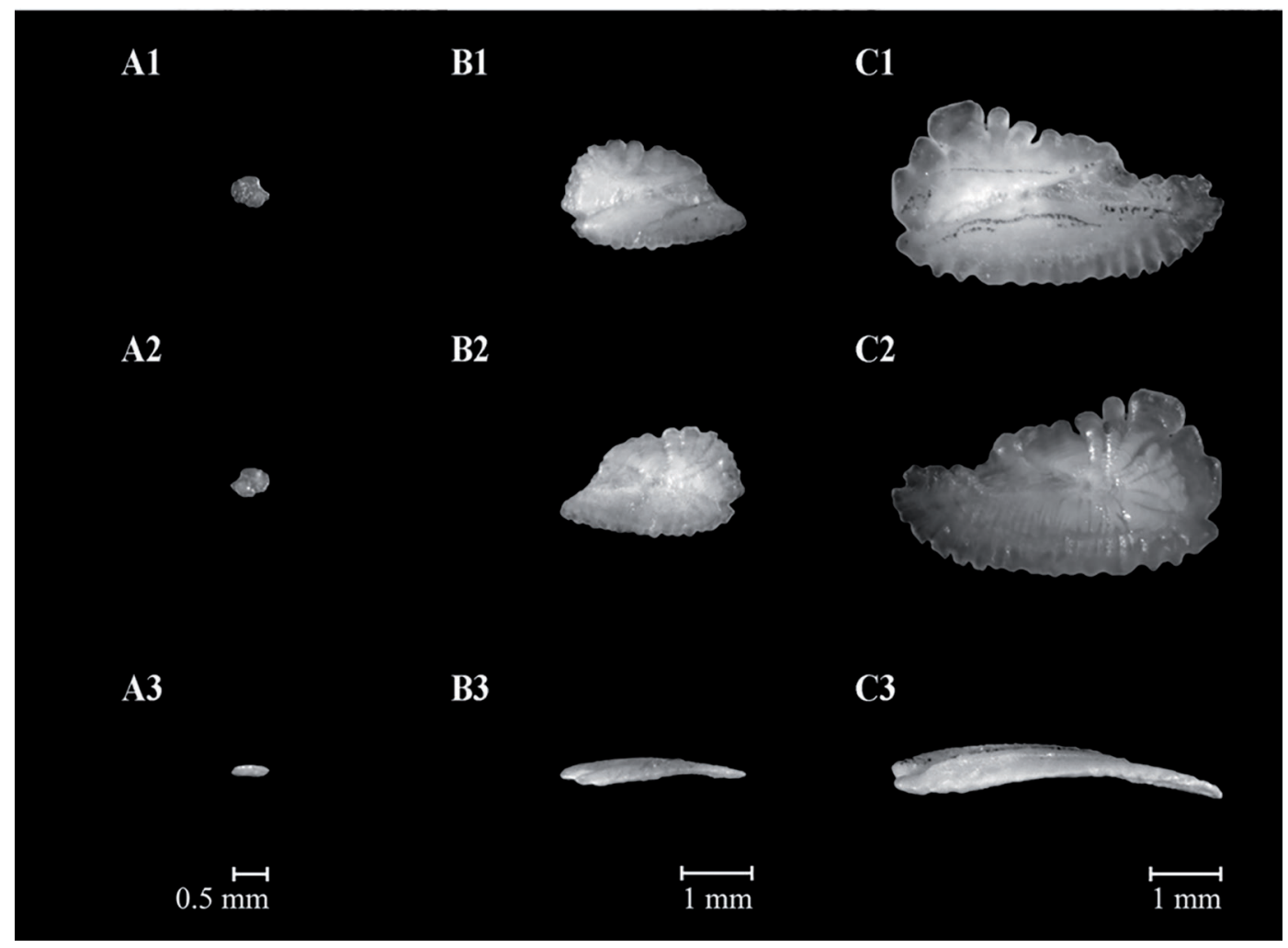

Plate 31. Illustrations (above) and photos (below) of Ariomma bondi otoliths from fish with total lengths: A. $17 \mathrm{~mm}$; B. $103 \mathrm{~mm}$; C. $191 \mathrm{~mm}$. The medial face is shown in A1, B1, C1; the lateral face in A2, B2, C2; and the ventral profile in A3, B3, C3 (Illustrations: Laura Montserrat; Photos: Cesar Santificetur). 
A1

A2

A3

थत्य

$\stackrel{\longmapsto}{2 \mathrm{~mm}}$

A1

A2

A3
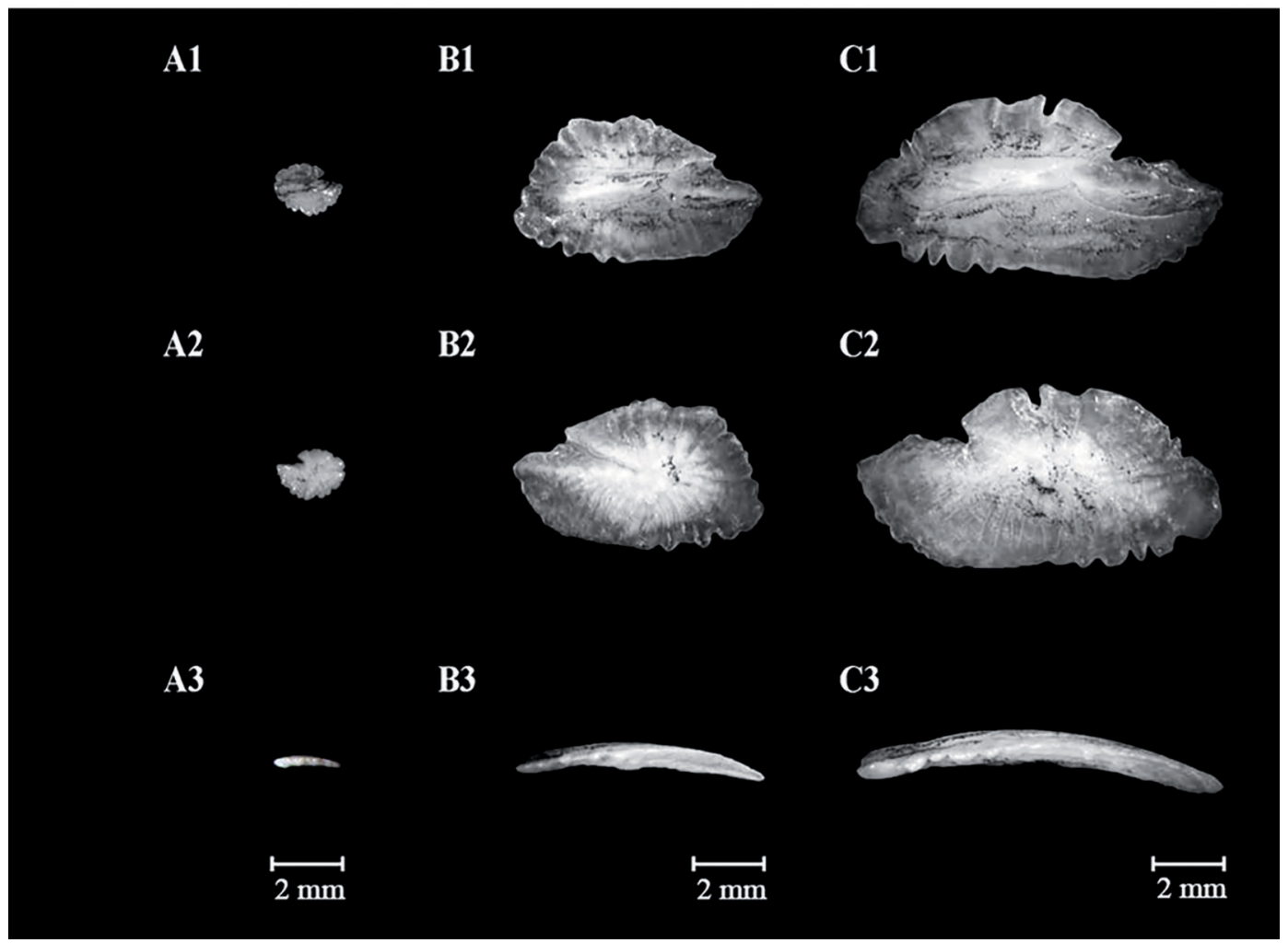

B1

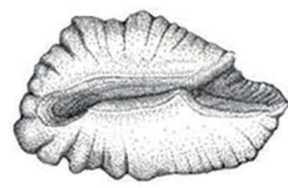

B2

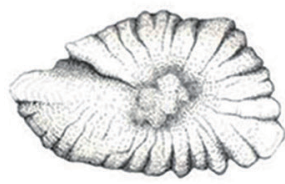

B3

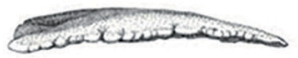

$\stackrel{\mathrm{mm}}{ }$
C1

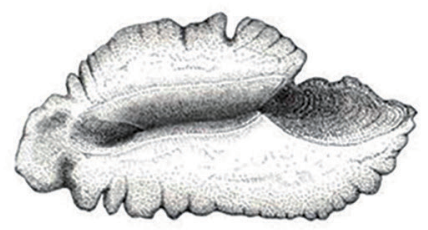

C2

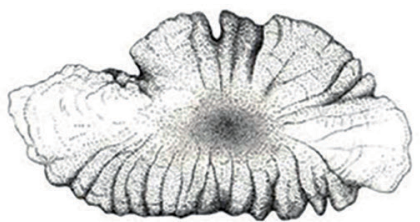

C3

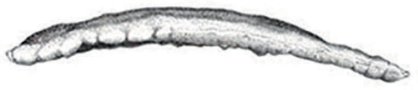

$\longmapsto$

Plate 32. Illustrations (above) and photos (below) of Peprilus paru otoliths from fish with total lengths: A. $37 \mathrm{~mm}$; B. $168 \mathrm{~mm}$; C. $301 \mathrm{~mm}$. The medial face is shown in A1, B1, C1; the lateral face in A2, B2, C2; and the ventral profile in A3, B3, C3 (Illustrations: Laura Montserrat; Photos: Cesar Santificetur). 
A1

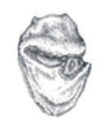

A2

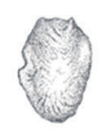

A3

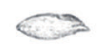

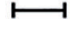

$2 \mathrm{~mm}$
B1

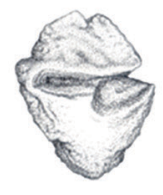

B2

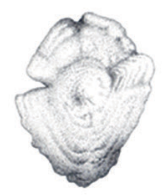

B3

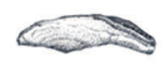

$\longmapsto$

$2 \mathrm{~mm}$
C1

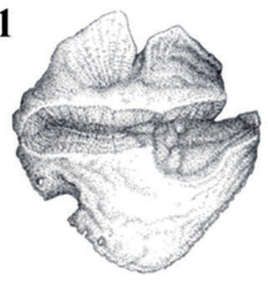

C2

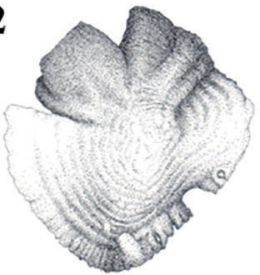

C3

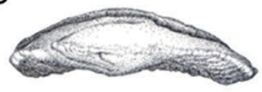

A1

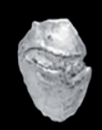

A2

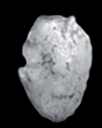

A3
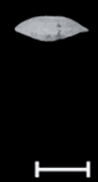

$2 \mathrm{~mm}$
B1

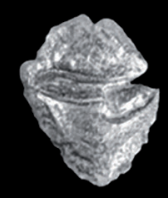

B2

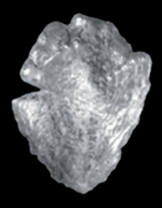

B3

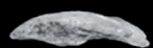

$2 \mathrm{~mm}$

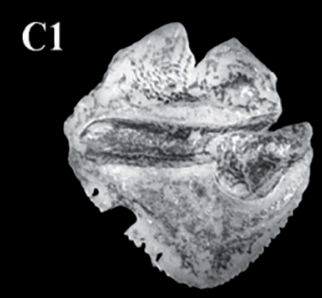

C2

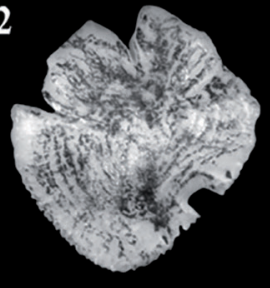

C3

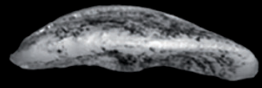

Plate 33. Illustrations (above) and photos (below) of Antigonia capros otoliths from fish with total lengths: A. 35 mm; B. 112 mm; C. $191 \mathrm{~mm}$. The medial face is shown in A1, B1, C1; the lateral face in A2, B2, C2; and the ventral profile in A3, B3, C3 (Illustrations and Photos: Alexandre Arackawa). 